\title{
Synthesis and Stereochemical Confirmation of the Secoiridoid Glucosides Nudiflosides D and A.
}

\author{
Stephen Hanessian,* Emily Mainetti, Fabien Lecomte. \\ Department of Chemistry, Université de Montréal \\ C.P. 6128, Succursale Centre-ville, \\ Montréal Québec H3C 3J7 Canada \\ stephen.hanessian@umontreal.ca
}

1- General information.

2- Experimental procedures of all compounds.

3- Selected ${ }^{1} \mathrm{H}$ and ${ }^{13} \mathrm{C}$ spectra for compounds $\mathbf{1}$ to $\mathbf{1 5}$ and ${ }^{31} \mathrm{P}$ for compound 5. ${ }^{1} \mathrm{H}$ NOESY for compounds 8 and 10; COSY, HMQC, HMBC for compounds $\mathbf{1}$ and $\mathbf{2}$. 


\section{1- General information:}

Solvents were purified with a solvent delivery system before use. All commercially available reagents were used without further purification. Oleuropein was purchased from Indofine. All reactions were performed under argon atmosphere. NMR $\left({ }^{1} \mathrm{H},{ }^{13} \mathrm{C}\right)$ spectra were recorded on Bruker AV-300, AV-400 RG and AV-500 spectrometers in $\mathrm{CDCl}_{3}$ or $\mathrm{CD}_{3} \mathrm{OD}$ or $\mathrm{D}_{2} \mathrm{O}$ with solvent resonance as the internal standard. Low and high-resolution mass spectra were recorded on VG Micromass, AEIMS 902, or Kratos MS-50 spectrometers using electrospray techniques. The purity of the target compounds was determined to be $>90 \%$ by LC/MS obtained on a Finnigan Surveyor MSQ spectrometer. Purity of the compounds was determined with an Alltech Prevail C18 column (250_ 4.6 $\mathrm{mm})$ at $0.5 \mathrm{~mL} / \mathrm{min}$ flow rate using a gradient of $20-80 \%$ acetonitrile-water $(0.1 \%$ trifluoroacetic acid). Optical rotations were recorded on a Perkin-Elmer 241 polarimeter in a $1 \mathrm{dm}$ cell at ambient temperature. Analytical thin-layer chromatography was performed on Merck 60F 254 precoated silica gel plates. Visualization was performed by $\mathrm{UV}$ or by development using $\mathrm{KMnO}_{4}$, ceric ammonium sulfate or $p$-anisaldehyde solutions. Flash column chromatography was performed using $(40-60 \mu \mathrm{m})$ silica gel at increased pressure.

\section{2- Experimental procedures:}

\section{2-Bromo-cyclopent-2-enone:}

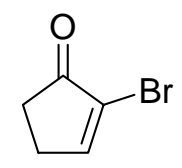

$\mathrm{C}_{5} \mathrm{H}_{5}$ BrO; F.W. $=160.00$ g.mol ${ }^{-1}$

To a cold $\left(0^{\circ} \mathrm{C}\right)$ solution of 2-cyclopenten-1-one (5 g, $60.90 \mathrm{mmol}, 1$ equiv.) in $30 \mathrm{~mL}$ of $\mathrm{CH}_{2} \mathrm{Cl}_{2}$ was added a solution of bromine (3.12 mL, $60.90 \mathrm{mmol}, 1$ equiv.) in $\mathrm{CH}_{2} \mathrm{Cl}_{2}(30 \mathrm{~mL})$. After $5 \mathrm{~min}$ stirring at $0{ }^{\circ} \mathrm{C}$, a solution of triethylamine $(12.73 \mathrm{~mL}$, 
$91.35 \mathrm{mmol}, 1.5$ equiv.) in $30 \mathrm{~mL}$ of $\mathrm{CH}_{2} \mathrm{Cl}_{2}$ was added. The reaction was then allowed to warm to room temperature. After a $2 \mathrm{~h}$ stirring, the mixture was filtered over a pad of celite and the solvents were evaporated. Purification of the crude by flash chromatography on silica gel (hexane/AcOEt : 9/1) afforded $8.19 \mathrm{~g}$ of the desired cyclopentenone (84\% yield).

${ }^{1} \mathrm{H}$ NMR $\left(\mathrm{CDCl}_{3} ; 300 \mathrm{MHz}\right) \delta 7.73(\mathrm{~m}, 1 \mathrm{H}), 2.65(\mathrm{~m}, 2 \mathrm{H}), 2.46(\mathrm{~m}, 2 \mathrm{H}) ;{ }^{13} \mathrm{C}$ NMR $\left(\mathrm{CDCl}_{3} ; 75 \mathrm{MHz}\right) \delta 201.6(\mathrm{C}), 161.9(\mathrm{CH}), 125.9(\mathrm{C}), 32.3\left(\mathrm{CH}_{2}\right), 27.9\left(\mathrm{CH}_{2}\right)$.

The spectra correspond to the ones previously described in the literature. ${ }^{1}$

\section{6-Bromo-1,4-dioxa-spiro[4.4]non-6-ene (3):}

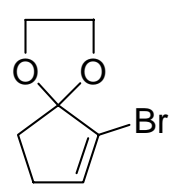

$\mathrm{C}_{7} \mathrm{H}_{9} \mathrm{BrO}_{2} ; \mathrm{F} . \mathrm{W} .=204.98{\mathrm{~g} . \mathrm{mol}^{-1}}^{-1}$

To a solution of 2-bromo-cyclopent-2-enone (3.06 g, $19.0 \mathrm{mmol}, 1$ equiv.) in benzene (150 mL) were added $p$-toluenesulfonic acid (18 mg, $0.095 \mathrm{mmol}, 0.005$ equiv.) and ethylene glycol (2.65 mL, $47.5 \mathrm{mmol}, 2.5$ equiv.). The mixture was refluxed with a Dean-Stark for $18 \mathrm{~h}$. After being cooled to room temperature, it was filtered over a pad of celite $/ \mathrm{Na}_{2} \mathrm{SO}_{4} /$ silica gel and the solvents were evaporated. Purification by flash chromatography on silica gel (hexane/AcOEt : 95/5) gave the desired ketal 3 in 67\% yield (2.62 g).

${ }^{1} \mathrm{H}$ NMR $\left(\mathrm{CDCl}_{3} ; 300 \mathrm{MHz}\right) \delta 6.16(\mathrm{t}, J=2 \mathrm{~Hz}, 1 \mathrm{H}), 4.18(\mathrm{~m}, 2 \mathrm{H}), 3.98(\mathrm{~m}, 2 \mathrm{H}), 2.36$ (m, 2H), 2.15 (m, 2H); ${ }^{13} \mathrm{C}$ NMR (CDCl $;$; 75 MHz) $\delta 136.5$ (CH), 123.7 (C), 117.5 (C), $65.8\left(2 \mathrm{CH}_{2}\right), 34.2\left(\mathrm{CH}_{2}\right), 28.5\left(\mathrm{CH}_{2}\right)$.

\footnotetext{
${ }^{1}$ Dunn, G.L.; DiPasquo, V.J.; Hoover, J.R.E. J. Org. Chem. 1968, 33, 1454.
} 
The spectra correspond to the ones previously described in the literature. ${ }^{2}$

\section{2-Benzyloxymethyl-cyclopent-2-enone (4):}

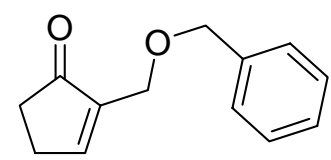

$\mathrm{C}_{13} \mathrm{H}_{14} \mathrm{O}_{2} ; \mathrm{F} . \mathrm{W} .=202.25$ g.mol ${ }^{-1}$

n-Butyllithium (1.6 M in hexanes, $30.3 \mathrm{~mL}, 48.53 \mathrm{mmol}, 1.2$ equiv.) was added to $100 \mathrm{~mL}$ of $\mathrm{THF}$ at $-78^{\circ} \mathrm{C}$. Then, a solution of ketal 3 (8.29 g, $40.44 \mathrm{mmol}, 1$ equiv.) in THF (60 mL) was added and the mixture was stirred at $-78{ }^{\circ} \mathrm{C}$ for $1 \mathrm{~h}$. Then, HMPA (14 mL, 80.88 mmol, 2 equiv.) and distilled BOMCl (19.7 mL, 141.54 mmol, 3.5 equiv.) were added at the same time via syringe. After a $1 \mathrm{~h}$ stirring at that temperature, the reaction was quenched by a saturated ammonium chloride solution. The aqueous layer was extracted with $\mathrm{Et}_{2} \mathrm{O}$. The combined organic phases were washed with brine and dried over $\mathrm{Na}_{2} \mathrm{SO}_{4}$. The crude was submitted to a rapid flash chromatography on silica gel (hexane/AcOEt : 95/5) to afforded the desired compound 4 contaminated by benzyl alcohol.

The amount of benzyl alcohol was calculated by ${ }^{1} \mathrm{H}$ NMR, then the mixture was diluted in $\mathrm{CH}_{2} \mathrm{Cl}_{2}(0.5 \mathrm{M})$ and triethylamine (2 equiv.), DMAP (0.3 equiv.) and tertbutyldimethylsilyl chloride (1 equiv.) were added at room temperature. After a 40 min stirring, the reaction was quenched by a saturated ammonium chloride solution. The aqueous layer was extracted with $\mathrm{Et}_{2} \mathrm{O}$. The combined organic phases were washed with brine and dried over $\mathrm{Na}_{2} \mathrm{SO}_{4}$. Purification by flash chromatography on silica gel (hexane/AcOEt : 9/1) afforded $7.7 \mathrm{~g}$ of the pure cyclopentenone 4 (94 \% yield).

\footnotetext{
${ }^{2}$ Smith, A.B.III; Branca, S.J.; Pilla, N.N.; Guaciaro, M.A. J. Org. Chem. 1982, 47, 1855-1869.
} 
IR (neat) 3060, 2900, 1700, 1640, 1450, 1370, 1260, 1200, 1110, $1030 \mathrm{~cm}^{-1} ;{ }^{1} \mathrm{H}$ NMR $\left(\mathrm{CDCl}_{3} ; 300 \mathrm{MHz}\right) \delta 7.64(\mathrm{~m}, 1 \mathrm{H}), 7.36(\mathrm{~m}, 5 \mathrm{H}), 4.59(\mathrm{~s}, 2 \mathrm{H}), 4.20(\mathrm{~m}, 2 \mathrm{H}), 2.63(\mathrm{~m}$,

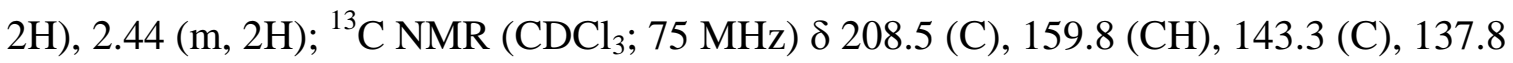
(C), $128.3(2 \mathrm{CH}), 127.6(2 \mathrm{CH}), 126.8(\mathrm{CH}), 73.1\left(\mathrm{CH}_{2}\right), 64.0\left(\mathrm{CH}_{2}\right), 34.8\left(\mathrm{CH}_{2}\right), 26.8$ $\left(\mathrm{CH}_{2}\right)$; HRMS (MNa ${ }^{+}$, calcd : 225.08915, found : 225.08833 .

\section{2-Benzyloxymethyl-3-[3-(1,3-dimethyl-2-oxo-octahydro-215- benzo[1,3,2]diazaphosphol-2-yl)-1-methyl-allyl]-cyclopentanone (5):}

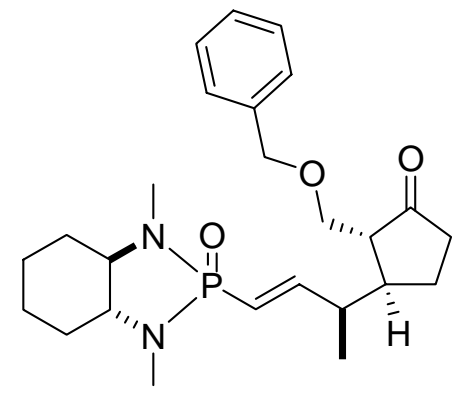

$\mathrm{C}_{25} \mathrm{H}_{37} \mathrm{~N}_{2} \mathrm{O}_{3} \mathrm{P} ; \mathrm{F} . \mathrm{W} .=444.55$ g.mol ${ }^{-1}$

To a cold $\left(-78{ }^{\circ} \mathrm{C}\right)$ solution of the phosphonamide $\mathbf{A}^{3}$ (965 mg, $3.98 \mathrm{mmol}, 1$ equiv.) in $35 \mathrm{~mL}$ of THF was added dropwise $n$-butyllithium (1.6 $\mathrm{M}$ in hexanes, $3 \mathrm{~mL}$, $4.78 \mathrm{mmol}, 1.2$ equiv.) and five minutes later was canulated a solution of cylopentenone 4 (966 mg, $4.78 \mathrm{mmol}, 1.2$ equiv.) in $7 \mathrm{~mL}$ of THF. After a $45 \mathrm{~min}$ stirring, the reaction was quenched by adding slowly $10 \mathrm{~mL}$ of water at $-78{ }^{\circ} \mathrm{C}$ and $5 \mathrm{~mL}$ of a saturated ammonium chloride solution. Then, the mixture was allowed to warm to room temperature. The layers were separated, the aqueous phase was extracted with AcOEt. The combined organic layers were dried over $\mathrm{Na}_{2} \mathrm{SO}_{4}$ and the solvents were evaporated.

\footnotetext{
${ }^{3}$ For preparation see: Hanessian, S.; Gomtsyan, A.; Malek, N. J. Org. Chem. 2000, 65, 5623.
} 
Purification by flash chromatography on silica gel (AcOEt/MeOH : 95/5) afforded $1.28 \mathrm{~g}$ of the desired product 5 (72 \% yield). ${ }^{4}$

$[\alpha]_{\mathrm{D}}^{20}-2.1^{\circ}$ (c $\left.0.95, \mathrm{CHCl}_{3}\right)$. IR (neat) 2900, 1740, 1620, 1450, 1370, 1240, 1170, 1010 $\mathrm{cm}^{-1} ;{ }^{1} \mathrm{H} \mathrm{NMR}\left(\mathrm{CDCl}_{3} ; 400 \mathrm{MHz}\right) \delta$ 7.36-7.26 (m, 5H), 6.66 (ddd, $J=20.3,16.6,8.7 \mathrm{~Hz}$, 1H), 5.42 (dd, $J=21.9,16.6 \mathrm{~Hz}, 1 \mathrm{H}$ ), 4.51 (d, $J=12.0 \mathrm{~Hz}, 1 \mathrm{H}$ ), 4.43 (d, $J=12.0 \mathrm{~Hz}$, 1H), 3.78 (dd, $J=9.4,4.1 \mathrm{~Hz}, 1 \mathrm{H}$ ), 3.63 (d, $J=9.4,3.3 \mathrm{~Hz}, 1 \mathrm{H}$ ), 2.76 (m, 1H), 2.61-2.31 (m, 5H), 2.50 (d, $J=11.8 \mathrm{~Hz}, 3 \mathrm{H}$ ), 2.41 (d, $J=11.8 \mathrm{~Hz}, 3 \mathrm{H}$ ), 2.17-2.01 (m, 5H), 1.83 (m, 2H), 1.51 (m, 1H), 1.26 (m, 3H), 1.14 (d, $J=6.8 \mathrm{~Hz}, 3 \mathrm{H}) ;{ }^{13} \mathrm{C} \mathrm{NMR}\left(\mathrm{CDCl}_{3} ; 75 \mathrm{MHz}\right) \delta$ 218.0 (C), 154.8 (CH), 138.0 (C), 128.1 (2 CH), 127.4 (2 CH), $127.3(\mathrm{CH}), 120.3$ (d, $J=$ $151.0 \mathrm{~Hz}, \mathrm{CH}), 73.1\left(\mathrm{CH}_{2}\right), 67.9\left(\mathrm{CH}_{2}\right), 64.5$ (d, $\left.J=7.2 \mathrm{~Hz}, \mathrm{CH}\right), 63.5$ (d, $J=5.2 \mathrm{~Hz}$, CH), $52.8(\mathrm{CH}), 43.5(\mathrm{CH}), 40.6$ (d, $J=18.0 \mathrm{~Hz}, \mathrm{CH}), 38.0\left(\mathrm{CH}_{2}\right), 28.6$ (d, $J=4.9 \mathrm{~Hz}, 2$ $\left.\mathrm{CH}_{3}\right)$, $28.4\left(\mathrm{CH}_{2}\right), 27.9\left(\mathrm{CH}_{2}\right), 24.0\left(2 \mathrm{CH}_{2}\right), 22.8\left(\mathrm{CH}_{2}\right), 18.1\left(\mathrm{CH}_{3}\right) ;{ }^{31} \mathrm{P} \mathrm{NMR}\left(\mathrm{CDCl}_{3}\right.$; $162 \mathrm{MHz}) \delta 32.68$ (s, 1P); HRMS $\left(\mathrm{MH}^{+}\right)$, calcd : 445.2620, found : 445.2630.

\section{2-Benzyloxymethyl-3-(2-hydroxy-1-methyl-ethyl)-cyclopentanol:}

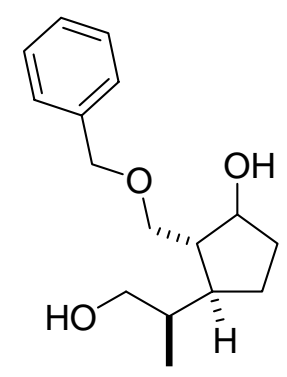

$\mathrm{C}_{16} \mathrm{H}_{24} \mathrm{O}_{3} ; \mathrm{F} . \mathrm{W} .=264.36$ g.mol ${ }^{-1}$

The phosphonamide 5 (1.73 g, 3.88 mmol, 1 equiv.) diluted in $\mathrm{CH}_{2} \mathrm{Cl}_{2}$ (40 mL) and methanol $(40 \mathrm{~mL})$ was cooled to $-78{ }^{\circ} \mathrm{C}$. Ozone was bubbled into, until a persistent

\footnotetext{
${ }^{4}$ When performed on a $3.88 \mathrm{~g}$ scale $(16 \mathrm{mmol})$, the yield was $62 \%$.
} 
blue color was perceived and no starting material was left (checked by TLC). The excess of ozone was then chased away by bubbling argon into the reaction vessel. Sodium borohydride (586 mg, $15.5 \mathrm{mmol}$, 4 equiv.) was then added at this temperature and the mixture was allowed to warm slowly to $0{ }^{\circ} \mathrm{C}$. After $1 \mathrm{~h}$ stirring at $0{ }^{\circ} \mathrm{C}$, the reaction was quenched with a saturated ammonium chloride solution for $30 \mathrm{~min}$. Then, the aqueous layer was extracted with $\mathrm{CH}_{2} \mathrm{Cl}_{2}$. The combined organic layers were washed with brine, dried over $\mathrm{Na}_{2} \mathrm{SO}_{4}$ and the solvents were removed under vacuum. Purification by flash chromatography on silica gel (hexane/AcOEt : 6/4) gave $983 \mathrm{mg}$ of the desired diol as a mixture of two diastereoisomers in a 2/1 ratio (96 \% yield). ${ }^{5}$

IR (neat) 3400, 3030, 2930, 1720, 1450, 1360, 1270, $1100 \mathrm{~cm}^{-1}$.

Major dias. : ${ }^{1} \mathrm{H}$ NMR (CDCl $\left.; 300 \mathrm{MHz}\right) \delta 7.34(\mathrm{~m}, 5 \mathrm{H}), 4.53\left(\mathrm{~m}_{\mathrm{AB}}, 2 \mathrm{H}\right), 4.30(\mathrm{~m}, 1 \mathrm{H})$, 3.67-3.39 (m, 4H), 2.10-1.78 (m, 4H), 1.70-1.50 (m, 2H), 1.31 (m, 1H), 0.90 (d, $J=6.9$ $\mathrm{Hz}, 3 \mathrm{H}) ;{ }^{13} \mathrm{C} \mathrm{NMR}\left(\mathrm{CDCl}_{3} ; 75 \mathrm{MHz}\right) \delta 137.8(\mathrm{C}), 128.3(2 \mathrm{CH}), 127.6(\mathrm{CH}), 127.5$ (2 CH), $77.6(\mathrm{CH}), 73.8\left(\mathrm{CH}_{2}\right), 73.2\left(\mathrm{CH}_{2}\right), 66.2\left(\mathrm{CH}_{2}\right), 49.7(\mathrm{CH}), 42.2(\mathrm{CH}), 39.1(\mathrm{CH})$, $33.3\left(\mathrm{CH}_{2}\right), 26.4\left(\mathrm{CH}_{2}\right), 14.6\left(\mathrm{CH}_{3}\right)$.

Minor dias. : ${ }^{1} \mathrm{H}$ NMR $\left(\mathrm{CDCl}_{3} ; 300 \mathrm{MHz}\right) \delta 7.34(\mathrm{~m}, 5 \mathrm{H}), 4.53\left(\mathrm{~m}_{\mathrm{AB}}, 2 \mathrm{H}\right), 4.02(\mathrm{~m}, 1 \mathrm{H})$, 3.62-3.35 (m, 4H), 1.95 (m, 1H), 1.83-1.57 (m, 5H), 1.26 (m, 1H), 0.92 (d, $J=6.7 \mathrm{~Hz}$, 3H); ${ }^{13} \mathrm{C}$ NMR (CDCl $; 75$ MHz) $\delta 137.6(\mathrm{C}), 128.4$ (2 CH), 127.7 (CH), $127.6(2 \mathrm{CH})$, $75.2(\mathrm{CH}), 73.3\left(\mathrm{CH}_{2}\right), 70.9\left(\mathrm{CH}_{2}\right), 66.0\left(\mathrm{CH}_{2}\right), 44.6(\mathrm{CH}), 41.4(\mathrm{CH}), 38.9(\mathrm{CH}), 33.7$ $\left(\mathrm{CH}_{2}\right), 26.4\left(\mathrm{CH}_{2}\right), 14.4\left(\mathrm{CH}_{3}\right)$.

HRMS (M+), calcd : 264.172545, found : 264.172538. MS (ESI) m/z: $\left(\mathrm{MH}^{+}\right)$265.2.

\footnotetext{
${ }^{5}$ When performed on a $4.43 \mathrm{~g}$ scale $(9.96 \mathrm{mmol})$, the yield was $81 \%$.
} 


\section{2-Benzyloxymethyl-3-[2-(tert-butyldiphenylsilanyloxy)-1-methyl-ethyl]- cyclopentanol (6):}

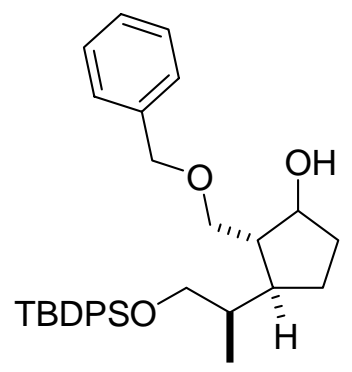

$\mathrm{C}_{32} \mathrm{H}_{42} \mathrm{O}_{3} \mathrm{Si} ; \mathrm{F} . \mathrm{W} .=502.77$ g.mol ${ }^{-1}$

To a $\mathrm{CH}_{2} \mathrm{Cl}_{2}(50 \mathrm{~mL})$ solution of the previous diol (2.58 g, $9.77 \mathrm{mmol}, 1$ equiv.) was added triethylamine (2.72 mL, $19.54 \mathrm{mmol}, 2$ equiv.), 4-DMAP (358 mg, 2.93 mmol, 0.3 equiv.) and tert-butyldiphenylsilyl chloride (2.54 mL, 9.77 mmol, 1 equiv.). After a $1 \mathrm{~h}$ stirring at room temperature, the reaction was quenched by a saturated ammonium chloride solution. The aqueous layer was extracted with $\mathrm{CH}_{2} \mathrm{Cl}_{2}$, the combined organic phases were washed with brine, dried over $\mathrm{Na}_{2} \mathrm{SO}_{4}$ and the solvents were evaporated. Purification by flash chromatography on silica gel (hexane/AcOEt : 9/1) gave $4.23 \mathrm{~g}$ of the desired protected alcohol 6 (86 \% yield).

IR (neat) 3450, 3070, 2900, 1720, 1590, 1470, 1430, 1270, $1100 \mathrm{~cm}^{-1}$.

Major dias. : ${ }^{1} \mathrm{H}$ NMR $\left(\mathrm{CDCl}_{3} ; 300 \mathrm{MHz}\right) \delta 7.65$ (m, 4H), 7.40-7.26 (m, 11H), 5.52 (d, J $=11.8 \mathrm{~Hz}, 1 \mathrm{H}), 4.44(\mathrm{~d}, J=11.8 \mathrm{~Hz}, 1 \mathrm{H}), 4.28(\mathrm{~m}, 1 \mathrm{H}), 3.66-3.40(\mathrm{~m}, 4 \mathrm{H}), 2.05-1.50(\mathrm{~m}$, 6H), 1.24 (m, 1H), 1.04 (s, 9H), 0.94 (d, $J=6.8 \mathrm{~Hz}, 3 \mathrm{H}) ;{ }^{13} \mathrm{C} \mathrm{NMR}\left(\mathrm{CDCl}_{3} ; 75 \mathrm{MHz}\right) \delta$ 137.9 (C), 135.5 (4 CH), 133.8 (2 C), 129.5 (2 CH), 128.3 (2 CH), 127.7 (CH), 127.5 (4 CH), $127.4(2 \mathrm{CH}), 75.2(\mathrm{CH}), 73.2\left(\mathrm{CH}_{2}\right), 70.8\left(\mathrm{CH}_{2}\right), 67.0\left(\mathrm{CH}_{2}\right), 45.4(\mathrm{CH}), 41.4$ (CH), $39.1(\mathrm{CH}), 33.8\left(\mathrm{CH}_{2}\right), 26.8\left(3 \mathrm{CH}_{3}\right), 26.5\left(\mathrm{CH}_{2}\right), 19.2(\mathrm{C}), 15.3\left(\mathrm{CH}_{3}\right)$. 
Minor dias. : ${ }^{1} \mathrm{H}$ NMR $\left(\mathrm{CDCl}_{3} ; 300 \mathrm{MHz}\right) \delta 7.65(\mathrm{~m}, 4 \mathrm{H}), 7.40-7.26(\mathrm{~m}, 11 \mathrm{H}), 5.53(\mathrm{~d}, J$ = $12.1 \mathrm{~Hz}, 1 \mathrm{H}), 4.45(\mathrm{~d}, J=12.1 \mathrm{~Hz}, 1 \mathrm{H}), 4.03(\mathrm{~m}, 1 \mathrm{H}), 3.66-3.40(\mathrm{~m}, 4 \mathrm{H}), 2.05-1.50(\mathrm{~m}$, 6H), 1.24 (m, 1H), 1.06 (s, 9H), 0.95 (d, $J=6.7 \mathrm{~Hz}, 3 \mathrm{H}) ;{ }^{13} \mathrm{C} \mathrm{NMR}\left(\mathrm{CDCl}_{3} ; 75 \mathrm{MHz}\right) \delta$ 138.2 (C), 135.5 (4 CH), 133.8 (2 C), 129.5 (2 CH), 128.3 (2 CH), 127.7 (CH), 127.5 (4 CH), $127.4(2 \mathrm{CH}), 78.4(\mathrm{CH}), 74.2\left(\mathrm{CH}_{2}\right), 73.2\left(\mathrm{CH}_{2}\right), 67.1\left(\mathrm{CH}_{2}\right), 50.1(\mathrm{CH}), 41.6$ (CH), $39.8(\mathrm{CH}), 33.0\left(\mathrm{CH}_{2}\right), 26.8\left(3 \mathrm{CH}_{3}\right), 26.5\left(\mathrm{CH}_{2}\right), 19.2(\mathrm{C}), 15.1\left(\mathrm{CH}_{3}\right)$.

HRMS $\left(\mathrm{MH}^{+}\right)$, calcd : 503.298149, found : 503.296800. MS (ESI) m/z: $\left(\mathrm{MH}^{+}\right)$503.4.

\section{2-Benzyloxymethyl-3-[2-(tert-butyldiphenylsilanyloxy)-1-methyl-ethyl]- cyclopentanone (7):}

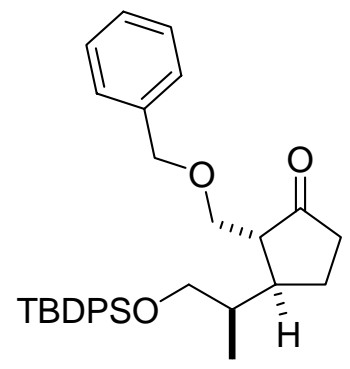

$\mathrm{C}_{32} \mathrm{H}_{40} \mathrm{O}_{3} \mathrm{Si} ; \mathrm{F} . \mathrm{W} .=500.75$ g.mol $^{-1}$

To a solution of the previous alcohol 6 ( $4.23 \mathrm{~g}, 8.41 \mathrm{mmol}, 1$ equiv.) in $140 \mathrm{~mL}$ of $\mathrm{CH}_{2} \mathrm{Cl}_{2}$ was added PCC (2.72 g, $12.6 \mathrm{mmol}, 1.5$ equiv.) and the reaction was stirred overnight at room temperature. The mixture was then filtered over a pad of celite/silica gel and the solvents were removed under vacuum. Purification by flash chromatography on silica gel yielded to $94 \%$ of the pure ketone 7 (3.98 g).

$[\alpha]_{\mathrm{D}}^{20}+17^{\circ}$ (c $0.4, \mathrm{CHCl}_{3}$ ). IR (neat) 3070, 2900, 1740, 1590, 1450, 1430, $1100 \mathrm{~cm}^{-1} ;{ }^{1} \mathrm{H}$ NMR (CDCl $;$; $400 \mathrm{MHz}) \delta 7.64$ (m, 4H), 7.39 (m, 6H), 7.29 (m, 5H), 4.57 (d, $J=12.1$ Hz, 1H), 4.39 (d, $J=12.1 \mathrm{~Hz}, 1 \mathrm{H}$ ), 3.80 (dd, $J=9.2,3.2 \mathrm{~Hz}, 1 \mathrm{H}$ ), 3.67 (dd, $J=10.2,5.4$ 
Hz, 1H), 3.55 (m, 2H), 2.39 (m, 1H), 2.28 (m, 1H), 2.12 (m, 2H), 2.00 (m, 1H), 1.85 (m, 1H), 1.48 (m, 1H), 1.05 (s, 9H), 1.01 (d, $J=6.9 \mathrm{~Hz}, 3 \mathrm{H}) ;{ }^{13} \mathrm{C} \mathrm{NMR}\left(\mathrm{CDCl}_{3} ; 100 \mathrm{MHz}\right) \delta$ 216.4 (C), 138.2 (C), 135.5 (4 CH), 133.6 (2 C), 129.6 (2 CH), 128.2 (2 CH), 127.6 (4 CH), $127.3(3 \mathrm{CH}), 73.1\left(\mathrm{CH}_{2}\right), 68.3\left(\mathrm{CH}_{2}\right), 66.6\left(\mathrm{CH}_{2}\right), 52.6(\mathrm{CH}), 40.7(\mathrm{CH}), 38.7$ ( $\left(\mathrm{CH}_{2}\right)$, $38.5(\mathrm{CH}), 26.8\left(3 \mathrm{CH}_{3}\right), 24.3\left(\mathrm{CH}_{2}\right), 19.1(\mathrm{C}), 15.5\left(\mathrm{CH}_{3}\right)$; HRMS $\left(\mathrm{MH}^{+}\right)$, calcd : 501.282499, found : 501.281581. MS (ESI) $\mathrm{m} / \mathrm{z}:\left(\mathrm{MH}^{+}\right)$501.4.

\section{5-Benzyloxymethyl-4-[2-(tert-butyldiphenylsilanyloxy)-1-methyl-ethyl]-cyclopent-2- enone (8):}

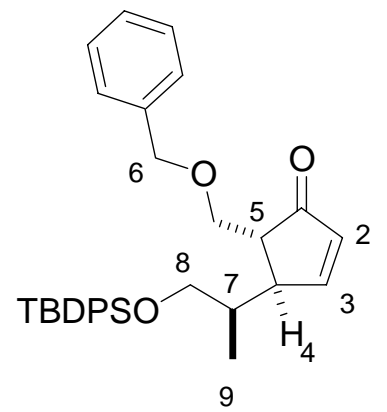

$\mathrm{C}_{32} \mathrm{H}_{38} \mathrm{O}_{3} \mathrm{Si} ; \mathrm{F} . \mathrm{W} .=498.74$ g.mol ${ }^{-1}$

Procedure A : For LDA $0.36 \mathrm{M}$ solution in THF : To a cold $\left(0{ }^{\circ} \mathrm{C}\right)$ solution of diisopropylamine (154 $\mu \mathrm{L}, 1.1 \mathrm{mmol}, 1.1$ equiv.) in $2 \mathrm{~mL}$ of THF was added dropwise $n$ butyllithium (1.6 M solution in hexanes, $625 \mu \mathrm{L}, 1 \mathrm{mmol}, 1$ equiv.) and the mixture was stirred at the same temperature for $30 \mathrm{~min}$.

To a cold $\left(-78^{\circ} \mathrm{C}\right)$ solution of ketone 7 (196 mg, $0.39 \mathrm{mmol}, 1$ equiv.) in $12 \mathrm{~mL}$ of THF was added freshly prepared LDA (0.36 M solution in THF, $1.3 \mathrm{~mL}, 0.47 \mathrm{mmol}, 1.2$ equiv.) and the mixture was stirred for $15 \mathrm{~min}$. Then chlorotrimethylsilane (60 $\mu \mathrm{L}, 0.47$ mmol, 1.2 equiv.) was added. After a 15 min stirring, the reaction was quenched with brine. The aqueous phase was extracted with $\mathrm{Et}_{2} \mathrm{O}$, the combined organic layers were 
dried over $\mathrm{Na}_{2} \mathrm{SO}_{4}$ and the solvents were evaporated. The crude was immediately diluted in $12 \mathrm{~mL}$ of dry acetonitrile and cooled to $0{ }^{\circ} \mathrm{C}$. Then, $\mathrm{Pd}(\mathrm{OAc})_{2}(87 \mathrm{mg}, 0.39 \mathrm{mmol}, 1$ equiv.) was added to the solution and the reaction was stirred $30 \mathrm{~min}$ at $0{ }^{\circ} \mathrm{C}$ and $2 \mathrm{~h}$ at room temperature. At that time, the palladium was turned black and the reaction stopped. The solvent was evaporated and the crude was directly submitted to flash chromatography on silica gel (hexane/AcOEt : 95/5) to give $99 \mathrm{mg}$ of the desired $\alpha, \beta$ insaturated ketone 8 ( $51 \%$ yield) and some remaining silylenol ether which was diluted in methanol, cooled to $0{ }^{\circ} \mathrm{C}$ and few drops of concentrated $\mathrm{HCl}$ were added; after usual work-up and flash chromatography on silica gel, we could isolate $47 \mathrm{mg}$ of the starting material 7 (24\%).

Procedure B : To a cold $\left(-78^{\circ} \mathrm{C}\right)$ solution of ketone 7 (3.97 g, $7.92 \mathrm{mmol}, 1$ equiv.) in $150 \mathrm{~mL}$ of THF was added dropwise LiHMDS (1 M solution in THF, 9.5mL, $9.5 \mathrm{mmol}$, 1.2 equiv.). After a 20 min stirring at this temperature, a solution of phenylselenenyl bromide (2.24 g, 9.5 mmol, 1.2 equiv.) in THF (15 mL) was added. The mixture was stirred 20 additional minutes at $-78{ }^{\circ} \mathrm{C}$ and then quenched with a saturated ammonium chloride solution. The aqueous layer was extracted with $\mathrm{Et}_{2} \mathrm{O}$. The combined organic phases were washed with brine, dried over $\mathrm{Na}_{2} \mathrm{SO}_{4}$ and the solvents were evaporated. The crude was submitted to a rapid flash chromatography on silica gel and the selenide product was then diluted in $80 \mathrm{~mL}$ of $\mathrm{CH}_{2} \mathrm{Cl}_{2}$. The mixture was cooled to $-30{ }^{\circ} \mathrm{C}$ and 8 $\mathrm{mL}$ of hydrogen peroxide (30 wt. \% solution in water) were added dropwise. The reaction was allowed to warm slowly to $-15{ }^{\circ} \mathrm{C}$ and after $30 \mathrm{~min}$ a white precipitate formed. The stirring was continued for $15 \mathrm{~min}$ and then the reaction was quenched with a saturated sodium bicarbonate solution. The aqueous layer was extracted with $\mathrm{Et}_{2} \mathrm{O}$, the combined 
organic phases were washed with brine, dried over $\mathrm{Na}_{2} \mathrm{SO}_{4}$ and the solvents were evaporated. Purification by flash chromatography on silica gel (hexane/AcOEt : 95/5) afforded $2.25 \mathrm{~g}$ of the unsaturated ketone 8 (57 \% yield) and $555 \mathrm{mg}$ of starting material 7 (14\%).

$[\alpha]_{\mathrm{D}}^{20}-22.2^{\circ}$ (c 0.825, $\mathrm{CHCl}_{3}$ ). IR (neat) 3070, 2900, 1710, 1590, 1450, 1360, $1100 \mathrm{~cm}^{-1}$; ${ }^{1} \mathrm{H}$ NMR $\left(\mathrm{CDCl}_{3} ; 300 \mathrm{MHz}\right) \delta 7.66(\mathrm{~m}, 5 \mathrm{H}), 7.40(\mathrm{~m}, 6 \mathrm{H}), 7.26(\mathrm{~m}, 5 \mathrm{H}), 6.17$ (dd, $J=$ 5.8, $2.1 \mathrm{~Hz}, 1 \mathrm{H}), 4.46$ (s, 2H), 3.79 (dd, $J=9.1,4.8 \mathrm{~Hz}, 1 \mathrm{H}), 3.64$ (dd, $J=9.1,3.6 \mathrm{~Hz}$, 1H), 3.60 (d, $J=5.7 \mathrm{~Hz}, 2 \mathrm{H}), 3.20(\mathrm{~m}, 1 \mathrm{H}), 2.30$ (m, 1H), 1.95 (m, 1H), 1.06 (s, 9H), $0.89(\mathrm{~d}, J=6.9 \mathrm{~Hz}, 3 \mathrm{H}) ;{ }^{13} \mathrm{C} \mathrm{NMR}\left(\mathrm{CDCl}_{3} ; 75 \mathrm{MHz}\right) \delta 209.7(\mathrm{C}), 167.6(\mathrm{CH}), 138.1(\mathrm{C})$, 135.4 (4 CH), 133.3 (CH), 133.2 (2 C), 129.6 (2 CH), 128.1 (2 CH), 127.6 (5 CH), 127.2 (2 $\mathrm{CH}), 73.0\left(\mathrm{CH}_{2}\right), 69.3\left(\mathrm{CH}_{2}\right), 67.0\left(\mathrm{CH}_{2}\right), 48.8(\mathrm{CH}), 47.5(\mathrm{CH}), 38.5(\mathrm{CH}), 26.8(3$ $\left.\mathrm{CH}_{3}\right), 19.1(\mathrm{C}), 13.9\left(\mathrm{CH}_{3}\right)$; HRMS $\left(\mathrm{MH}^{+}\right)$, calcd : 499.266849, found : 499.265000. MS (ESI) $\mathrm{m} / \mathrm{z}:\left(\mathrm{MH}^{+}\right) 499.4$.

Significant ${ }^{1} \mathrm{H}$ NOESY correlations: $\mathrm{H}_{2}-6 \Leftrightarrow \mathrm{H}-4 ; \mathrm{H}-5 \Leftrightarrow \mathrm{H}_{2}-8 ; \mathrm{H}-5 \Leftrightarrow \mathrm{H}-7 ; \mathrm{H}-5 \Leftrightarrow \mathrm{H}_{3}-9$

3-Benzyloxymethyl-4-[2-(tert-butyldiphenylsilanyloxy)-1-methyl-ethyl]-6-oxabicyclo[3.1.0]hexan-2-one (9):

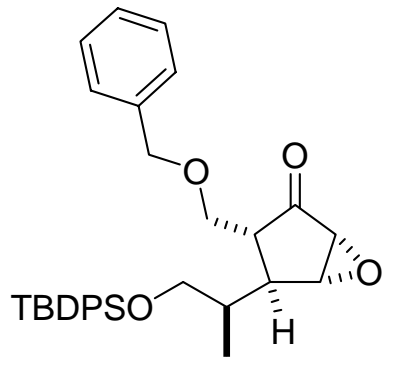

$\mathrm{C}_{32} \mathrm{H}_{38} \mathrm{O}_{4} \mathrm{Si} ; \mathrm{F} . \mathrm{W} .=514.73$ g.mol ${ }^{-1}$

To a cold $\left(-10^{\circ} \mathrm{C}\right)$ solution of ketone 8 (486 mg, $0.97 \mathrm{mmol}, 1$ equiv.) in $13 \mathrm{~mL}$ of methanol was added $515 \mu \mathrm{L}$ of hydrogen peroxide (30 wt. \% solution in water) and 0.1 
$\mathrm{mL}$ of a $1 \mathrm{M}$ water solution of potassium hydroxide. After a $2 \mathrm{~h}$ stirring at this temperature, the reaction was quenched with saturated ammonium chloride solution. The aqueous phase was extracted with $\mathrm{CH}_{2} \mathrm{Cl}_{2}$, the combined organic layers were washed with brine, dried over $\mathrm{Na}_{2} \mathrm{SO}_{4}$ and the solvents were removed under vacuum. Purification by flash chromoatography on silica gel (hexane/AcOEt : 95/5) afforded $399 \mathrm{mg}$ of the epoxide 9 as a mixture of two diastereoisomers in a 9/1 ratio (80\% yield). ${ }^{6}$

$[\alpha]_{\mathrm{D}}^{20}+9.1^{\circ}$ (c $\left.0.175, \mathrm{CHCl}_{3}\right)$. IR (neat) 3070, 2900, 1745, 1590, 1470, 1360, $1100 \mathrm{~cm}^{-1}$;

${ }^{1} \mathrm{H}$ NMR (CDCl $\left.; 300 \mathrm{MHz}\right) \delta 7.63(\mathrm{~m}, 4 \mathrm{H}), 7.40$ (m, 6H), 7.26 (m, 5H), 4.45 (s, 2H), 3.86 (d, $J=2.3 \mathrm{~Hz}, 1 \mathrm{H}), 3.58(\mathrm{~m}, 4 \mathrm{H}), 3.38$ (d, $J=2.0 \mathrm{~Hz}, 1 \mathrm{H}), 2.60$ (dd, $J=5.5,2.4 \mathrm{~Hz}$, 1H), 2.38 (ddd, $J=8.1,6.0,2.2 \mathrm{~Hz}, 1 \mathrm{H}$ ), 1.91 (sept, $J=6.4 \mathrm{~Hz}, 1 \mathrm{H}$ ), 1.05 (s, 9H), 0.90 $(\mathrm{d}, J=6.4 \mathrm{~Hz}, 3 \mathrm{H}) ;{ }^{13} \mathrm{C} \mathrm{NMR}\left(\mathrm{CDCl}_{3} ; 75 \mathrm{MHz}\right) \delta 208.3$ (C), 137.9 (C), 135.4 (4 CH), 133.2 (2 C), 129.6 (2 CH), 128.2 (2 CH), 127.6 (5 CH), $127.3(2 \mathrm{CH}), 72.7\left(\mathrm{CH}_{2}\right), 71.6$ $\left(\mathrm{CH}_{2}\right)$, $66.4\left(\mathrm{CH}_{2}\right), 60.4(\mathrm{CH}), 55.9(\mathrm{CH}), 48.5(\mathrm{CH}), 41.9(\mathrm{CH}), 37.2(\mathrm{CH}), 26.7\left(3 \mathrm{CH}_{3}\right)$, 19.1 (C), $13.7\left(\mathrm{CH}_{3}\right)$; MS (ESI) m/z: $\left(\mathrm{MH}^{+}\right)$515.4, $\left(\mathrm{MNa}^{+}\right)$537.4 ; HRMS (MH+) calcd : 515.26176, found : 515.26074 $\left(\mathrm{MNa}^{+}\right)$, calcd : 537.24370, found : 537.24339.

\section{2-Benzyloxymethyl-3-[2-(tert-butyldiphenylsilanyloxy)-1-methyl-ethyl]-4-hydroxy- cyclopentanone (10):}

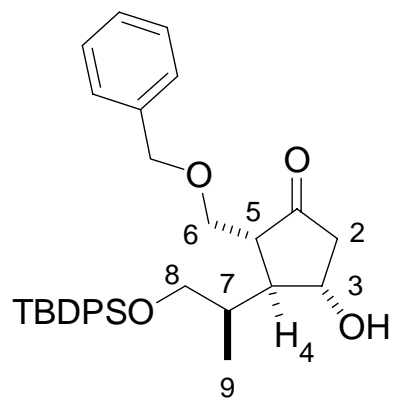

\footnotetext{
${ }^{6}$ When performed on a $2.49 \mathrm{~g}$ scale $(4.99 \mathrm{mmol})$, the yield was $73 \%$.
} 
$\mathrm{C}_{32} \mathrm{H}_{40} \mathrm{O}_{4} \mathrm{Si} ; \mathrm{F} . \mathrm{W} .=516.75$ g.mol ${ }^{-1}$

To a solution of diphenyldiselenide $(1.1 \mathrm{~g}, 3.51 \mathrm{mmol}, 1.5$ equiv.) in $20 \mathrm{~mL}$ of ethanol was added portionwise sodium borohydride (266 mg, $7.02 \mathrm{mmol}$, 3 equiv.). When the emission of hydrogen stopped and all sodium borohydride was dissolved, the pale yellow solution was cooled to $0{ }^{\circ} \mathrm{C}$ and acetic acid (67 $\mu \mathrm{L}, 1.17 \mathrm{mmol}, 0.5$ equiv.) was added. After a 5 min stirring, this mixture was added via canula to a cold $\left(0{ }^{\circ} \mathrm{C}\right)$ solution of epoxide 9 (1.21 g, $2.34 \mathrm{mmol}, 1$ equiv.) in $10 \mathrm{~mL}$ of ethanol. After $5 \mathrm{~min}$ the reaction was over. The mixture is then diluted with AcOEt and $\mathrm{O}_{2}$ was bubbled into for 3 min. The organic layer was washed twice with brine. The aqueous phase is extracted with AcOEt, the combined organic layers were dried over $\mathrm{Na}_{2} \mathrm{SO}_{4}$ and the solvents were removed under vacuum. Purification by flash chromatography on silica gel (hexane/AcOEt : 9/1 $\rightarrow 7 / 3$ ) afforded $163 \mathrm{mg}$ of the insaturated ketone 8 (14 \%) and 900 mg of the desired alcohol 10 (74 \% yield).

$[\alpha]_{\mathrm{D}}^{20}+7.8^{\circ}$ (c $0.245, \mathrm{CHCl}_{3}$ ). IR (neat) 3450, 3070, 2900, 1745, 1590, 1470, 1430, 1360, 1240, $1100 \mathrm{~cm}^{-1} ;{ }^{1} \mathrm{H}$ NMR (CDCl $\left.; 400 \mathrm{MHz}\right) \delta 7.67$ (m, 4H), 7.45 (m, 6H), 7.30 (m, 5H), 4.54 (d, $J=11.8 \mathrm{~Hz}, 1 \mathrm{H}), 4.45$ (d, $J=11.8 \mathrm{~Hz}, 1 \mathrm{H}), 4.25$ (m, 1H), 3.92 (dd, $J=$ 8.7, $2.8 \mathrm{~Hz}, 1 \mathrm{H}$ ), 3.64 (d, $J=6.0 \mathrm{~Hz}, 2 \mathrm{H}), 3.51$ (dd, $J=8.7,3.2 \mathrm{~Hz}, 1 \mathrm{H}), 2.64$ (dd, $J=$ 18.5, $5.8 \mathrm{~Hz}, 1 \mathrm{H}$ ), 2.41 (m, 1H), 2.38 (dd, $J=18.5,5.3 \mathrm{~Hz}, 1 \mathrm{H}), 2.22$ (m, 1H), 1.87 (sept, $J=6.6 \mathrm{~Hz}, 1 \mathrm{H}), 1.08(\mathrm{~s}, 9 \mathrm{H}), 0.85(\mathrm{~d}, J=6.6 \mathrm{~Hz}, 3 \mathrm{H}) ;{ }^{13} \mathrm{C} \mathrm{NMR}\left(\mathrm{CDCl}_{3} ; 75 \mathrm{MHz}\right) \delta$ 217.5 (C), 137.1 (C), 135.5 (4 CH), 133.0 (2 C), 129.7 (2 CH), 128.3 (2 CH), 127.8 (4 CH), $127.7(\mathrm{CH}), 127.6(2 \mathrm{CH}), 73.5\left(\mathrm{CH}_{2}\right), 71.2(\mathrm{CH}), 69.7\left(\mathrm{CH}_{2}\right), 67.9\left(\mathrm{CH}_{2}\right), 51.9$ (CH), $50.8(\mathrm{CH}), 48.3\left(\mathrm{CH}_{2}\right), 38.6(\mathrm{CH}), 26.7\left(3 \mathrm{CH}_{3}\right), 19.1(\mathrm{C}), 14.8\left(\mathrm{CH}_{3}\right)$; MS (ESI) $m / z:\left(\mathrm{MH}^{+}\right) 517.4$. 
Significant ${ }^{1} \mathrm{H}$ NOESY correlations: $\mathrm{H}-5 \Leftrightarrow \mathrm{H}-3 ; \mathrm{H}-3 \Leftrightarrow \mathrm{H}_{3}-9 ; \mathrm{H}-4 \Leftrightarrow \mathrm{H}_{2}-6$

\section{3-Benzyloxymethyl-2-[2-(tert-butyldiphenylsilanyloxy)-1-methyl-ethyl]-4-methylene- cyclopentanol (11):}

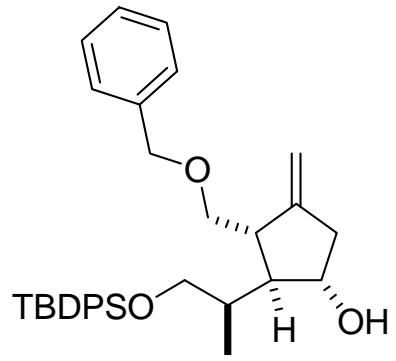

$\mathrm{C}_{33} \mathrm{H}_{42} \mathrm{O}_{3} \mathrm{Si} ; \mathrm{F} . \mathrm{W} .=514.78$ g.mol ${ }^{-1}$

Under argon and in a dried flask was weighted Nysted reagent (20 wt. \% suspension in THF, $363 \mathrm{mg}, 0.158 \mathrm{mmol}, 2$ equiv.) and $0.5 \mathrm{~mL}$ of THF was added. The suspension was cooled to $0{ }^{\circ} \mathrm{C}$ and titanium(IV) chloride (17 $\mu \mathrm{L}, 0.158 \mathrm{mmol}, 2$ equiv.) was added dropwise. When the emission of fume stopped, a solution of the hydroxyketone 10 (41 mg, 0.079 mmol, 1 equiv.) was added via canula. The mixture was then allowed to warm to room temperature and stirred for $1 \mathrm{~h}$. The suspension was then filtered over a pad of celite/silica gel and the solvents were evaporated. Purification by flash chromatography (hexane/AcOEt : 9/1 $\rightarrow 7 / 3$ ) gave $30 \mathrm{mg}$ of the desired alkene 11 (74 \% yield). ${ }^{7}$

$[\alpha]_{\mathrm{D}}^{20}+13^{\circ}\left(\mathrm{c} 0.2, \mathrm{CHCl}_{3}\right)$. IR (neat) 3440, 3070, 2900, 1660, 1590, 1470, 1430, 1390, 1360, $1100 \mathrm{~cm}^{-1} ;{ }^{1} \mathrm{H}$ NMR $\left(\mathrm{CDCl}_{3} ; 300 \mathrm{MHz}\right) \delta 7.68(\mathrm{~m}, 4 \mathrm{H}), 7.42$ (m, 6H), 7.28 (m, 5H), 5.02 (s, 1H), 4.94 (s, 1H), 4.52 (s, 2H), 4.03 (m, 1H), 3.63 (m, 3H), 3.48 (dd, $J=$ 8.9, $5.3 \mathrm{~Hz}, 1 \mathrm{H}$ ), 2.61 (dd, $J=15.4,5.8 \mathrm{~Hz}, 1 \mathrm{H}), 2.44$ (m, 2H), 1.86 (m, 1H), 1.72 (sept, $J=6.7 \mathrm{~Hz}, 1 \mathrm{H}), 1.08(\mathrm{~s}, 9 \mathrm{H}), 0.85(\mathrm{~d}, J=6.7 \mathrm{~Hz}, 3 \mathrm{H}) ;{ }^{13} \mathrm{C} \mathrm{NMR}\left(\mathrm{CDCl}_{3} ; 75 \mathrm{MHz}\right) \delta$

\footnotetext{
${ }^{7}$ When performed on a $318 \mathrm{mg}$ scale $(0.615 \mathrm{mmol})$, the yield was $69 \%$.
} 
150.6 (C), 138.0 (C), 135.5 (4 CH), 133.1 (2 C), 129.6 (2 CH), 128.2 (2 CH), 127.6 (4 $\mathrm{CH}), 127.4(3 \mathrm{CH}), 107.7\left(\mathrm{CH}_{2}\right), 74.8(\mathrm{CH}), 74.1\left(\mathrm{CH}_{2}\right), 73.2\left(\mathrm{CH}_{2}\right), 68.3\left(\mathrm{CH}_{2}\right), 54.0$ (CH), $46.3(\mathrm{CH}), 42.8\left(\mathrm{CH}_{2}\right), 38.8(\mathrm{CH}), 26.7\left(3 \mathrm{CH}_{3}\right), 19.1(\mathrm{C}), 15.2\left(\mathrm{CH}_{3}\right)$; MS (ESI) m/z: $\left(\mathrm{MH}^{+}\right)$515.4, $\left(\mathrm{MNa}^{+}\right)$537.4 ; HRMS $\left(\mathrm{MH}^{+}\right)$calcd : 515.29815, found : 515.29847 $\left(\mathrm{MNa}^{+}\right)$, calcd : 537.28009, found : 537.28009.

\section{2-[2-(tert-Butyldiphenylsilanyloxy)-1-methyl-ethyl]-3-hydroxymethyl-4-methylene- cyclopentanol:}

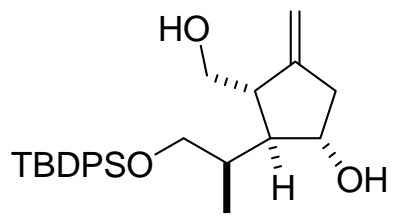

$\mathrm{C}_{26} \mathrm{H}_{36} \mathrm{O}_{3} \mathrm{Si} ; \mathrm{F} . \mathrm{W} .=424.65$ g.mol ${ }^{-1}$

To a cold $\left(-40^{\circ} \mathrm{C}\right)$ solution of benzylated alcohol 11 (87 mg, $0.17 \mathrm{mmol}, 1$ equiv.) in $2 \mathrm{~mL}$ of $\mathrm{CH}_{2} \mathrm{Cl}_{2}$ was added dropwise boron trichloride (1 M in heptane, $423 \mu \mathrm{L}, 0.42$ mmol, 2.5 equiv.). The mixture was stirred at this temperature for $30 \mathrm{~min}$ and then quenched by a solution of triethylamine $(0.4 \mathrm{~mL})$ and methanol $(1.2 \mathrm{~mL})$. The reaction was then allowed to warm to room temperature. It was washed with brine, the aqueous phase was extracted with AcOEt and the combined organic layers were dried over $\mathrm{Na}_{2} \mathrm{SO}_{4}$. After evaporation of the solvents, the crude was purified by flash chromatography on silica gel (hexane/AcOEt : 8/2 $\rightarrow 7 / 3$ ) to afford $53 \mathrm{mg}$ of the desired diol (73\% yield).

$[\alpha]_{\mathrm{D}}^{20}+18^{\circ}$ (c $0.1, \mathrm{CHCl}_{3}$ ). IR (neat) 3380, 3070, 2900, 1660, 1590, 1470, 1430, 1250, $1100 \mathrm{~cm}^{-1} ;{ }^{1} \mathrm{H}$ NMR (CDCl $;$; $\left.400 \mathrm{MHz}\right) \delta 7.70$ (m, 4H), 7.45 (m, 6H), 5.07 (s, 1H), 4.94 (s, 1H), 4.08 (m, 1H), 3.74 (dd, $J=10.8,4.7 \mathrm{~Hz}, 1 \mathrm{H}), 3.65$ (m, 2H), 3.54 (dd, $J=10.8$, 
$6.9 \mathrm{~Hz}, 1 \mathrm{H}), 2.64$ (dd, $J$ = 15.5, $6.2 \mathrm{~Hz}, 1 \mathrm{H}), 2.44$ (m, 2H), 1.77 (m, 2H), 1.10 (s, 9H),

$0.85(\mathrm{~d}, J=6.5 \mathrm{~Hz}, 3 \mathrm{H}) ;{ }^{13} \mathrm{C} \mathrm{NMR}\left(\mathrm{CDCl}_{3} ; 100 \mathrm{MHz}\right) \delta 150.2(\mathrm{C}), 136.0(4 \mathrm{CH}), 133.5$

(2 C), $130.3(2 \mathrm{CH}), 128.2(4 \mathrm{CH}), 108.8\left(\mathrm{CH}_{2}\right), 75.3(\mathrm{CH}), 69.0\left(\mathrm{CH}_{2}\right), 66.4\left(\mathrm{CH}_{2}\right), 53.9$

(CH), $48.9(\mathrm{CH}), 42.9\left(\mathrm{CH}_{2}\right), 39.0(\mathrm{CH}), 27.3\left(3 \mathrm{CH}_{3}\right), 19.6(\mathrm{C}), 15.5\left(\mathrm{CH}_{3}\right)$; MS (ESI)

m/z: $\left(\mathrm{MH}^{+}\right)$425.2, $\left(\mathrm{MH}^{+}-\mathrm{H}_{2} \mathrm{O}\right)$ 407.2 ; HRMS $\left(\mathrm{MNa}^{+}\right)$, calcd : 447.23314, found :

447.23388.

\section{2-[2-(tert-Butyl-diphenyl-silanyloxy)-1-methyl-ethyl]-3-hydroxymethyl-4-methyl-} cyclopentanol (12):

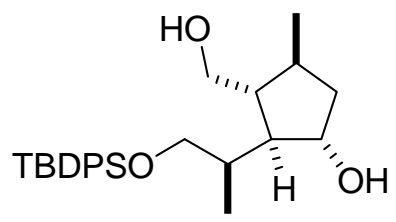

$\mathrm{C}_{26} \mathrm{H}_{38} \mathrm{O}$ S Si; F.W. $=426.67$ g.mol ${ }^{-1}$

A solution of the previous compound ( $57 \mathrm{mg}, 0.13 \mathrm{mmol}, 1$ equiv.) in $8.5 \mathrm{~mL}$ of $\mathrm{CH}_{2} \mathrm{Cl}_{2}$ under argon was frozen with liquid nitrogen. The frozen mixture was put under vacuum and then under argon again. The Crabtree catalyst, $\left[\operatorname{Ir}(\operatorname{cod}) \mathrm{py}\left(\mathrm{PCy}_{3}\right)\right] \mathrm{PF}_{6},(12$ mg, $0.015 \mathrm{mmol}, 0.1$ equiv.) was then added. The vacuum was made again and then the reaction was put under hydrogen. The solvent was rapidly warmed up with a water bath and stirring began. The orange solution became yellow after $3 \mathrm{~min}$. The reaction was allowed to stir at room temperature for 2 hours, after checking the completion by TLC. The solvent was evaporated and the crude was directly submitted to flash chromatography on silica gel (hexane/AcOEt : $8 / 2 \rightarrow 7 / 3$ ) to afford $41 \mathrm{mg}$ of the desired diol 12 as a unique diastereomer (74\% yield). 
$[\alpha]_{\mathrm{D}}^{20}+5.6^{\circ}$ (c $0.25, \mathrm{CHCl}_{3}$ ). IR (neat) 3370, 3070, 2900, 1590, 1470, 1430, $1100 \mathrm{~cm}^{-1}$;

${ }^{1} \mathrm{H}$ NMR (CDCl $;$; $\left.400 \mathrm{MHz}\right) \delta 7.68$ (m, 4H), 7.43 (m, 6H), 4.06 (m, 1H), 3.61 (m, 3H), 3.55 (dd, $J=10.6,5.6$ Hz, 1H), 2.08 (m, 1H), 1.81-1.70 (m, 3H), 1.48-1.38 (m, 2H), 1.07 (s, 9H), 1.01 (d, $J=6.8 \mathrm{~Hz}, 3 \mathrm{H}), 0.83(\mathrm{~d}, J=6.7 \mathrm{~Hz}, 3 \mathrm{H}) ;{ }^{13} \mathrm{C} \mathrm{NMR}\left(\mathrm{CDCl}_{3} ; 100 \mathrm{MHz}\right) \delta$ $135.5(4 \mathrm{CH}), 133.2(2 \mathrm{C}), 129.6(2 \mathrm{CH}), 127.6(4 \mathrm{CH}), 76.1(\mathrm{CH}), 67.7\left(\mathrm{CH}_{2}\right), 65.4$ ( $\left(\mathrm{CH}_{2}\right), 53.4(\mathrm{CH}), 50.3(\mathrm{CH}), 42.8\left(\mathrm{CH}_{2}\right), 38.0(\mathrm{CH}), 33.4(\mathrm{CH}), 26.7\left(3 \mathrm{CH}_{3}\right), 20.0$ ( $\left(\mathrm{CH}_{3}\right), 19.1(\mathrm{C}), 14.4\left(\mathrm{CH}_{3}\right)$; HRMS $\left(\mathrm{MH}^{+}\right)$, calcd : 427.26630, found : 427.26655. MS (ESI) $\mathrm{m} / \mathrm{z}:\left(\mathrm{MH}^{+}\right)$427.2, $\left(\mathrm{MH}^{+}-\mathrm{H}_{2} \mathrm{O}\right)$ 409.2, $\left(\mathrm{MNa}^{+}\right)$449.2.

3-Hydroxymethyl-2-(2-hydroxy-1-methyl-ethyl)-4-methyl-cyclopentanol (13):

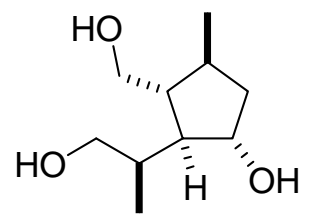

$\mathrm{C}_{10} \mathrm{H}_{20} \mathrm{O}_{3}$; F.W. $=188.27$ g.mol ${ }^{-1}$

The silylated alcohol 12 (30 mg, $0.070 \mathrm{mmol}, 1$ equiv.) was dissolved in $4 \mathrm{~mL}$ of THF, cooled to $0{ }^{\circ} \mathrm{C}$ and $\mathrm{Bu}_{4} \mathrm{NF}$ ( $1 \mathrm{M}$ solution in THF, $70 \mu \mathrm{L}, 0.070 \mathrm{mmol}$, 1 equiv.) was added. After a 30 min stirring at room temperature, the reaction was quenched with a saturated ammonium chloride solution. The aqueous phase was extracted with AcOEt and the combined organic layers were dried over $\mathrm{Na}_{2} \mathrm{SO}_{4}$. Purification by flash chromatography on silica gel (AcOEt/MeOH : 9/1) gave $12 \mathrm{mg}$ of the desired triol 13 as a clear viscous oil (91\% yield).

$[\alpha]_{\mathrm{D}}{ }^{20}+16^{\circ}$ (c $\left.0.275, \mathrm{CH}_{3} \mathrm{OH}\right)$ [litt. $[\alpha]_{\mathrm{D}}{ }^{28}+11^{\circ}$ (c $\left.\left.0.30, \mathrm{CH}_{3} \mathrm{OH}\right)\right]$. IR (neat) 3293 (broad), 2925, 1452, 1378, 1029, 734, $700 \mathrm{~cm}^{-1}$; 
${ }^{1} \mathrm{H}$ NMR $\left(\mathrm{CD}_{3} \mathrm{OD} ; 300 \mathrm{MHz}\right) \delta 4.03(\mathrm{q}, J=4.6 \mathrm{~Hz}, 1 \mathrm{H}), 3.53(\mathrm{~m}, 3 \mathrm{H}), 3.41$ (dd, $J=$ 10.9, $6.8 \mathrm{~Hz}, 1 \mathrm{H}), 2.01$ (sept, $J=7.8 \mathrm{~Hz}, 1 \mathrm{H}), 1.75$ (m, 2H), 1.62 (m, 1H), 1.46 (m, 2H), $1.03(\mathrm{~d}, J=6.8 \mathrm{~Hz}, 3 \mathrm{H}), 0.93(\mathrm{~d}, J=6.8 \mathrm{~Hz}, 3 \mathrm{H}) ;{ }^{13} \mathrm{C}$ NMR $\left(\mathrm{CD}_{3} \mathrm{OD} ; 75 \mathrm{MHz}\right) \delta 76.8$ (CH), $67.1\left(\mathrm{CH}_{2}\right), 66.8\left(\mathrm{CH}_{2}\right), 54.2(\mathrm{CH}), 51.3(\mathrm{CH}), 43.7\left(\mathrm{CH}_{2}\right), 39.3(\mathrm{CH}), 35.7(\mathrm{CH})$, $20.9\left(\mathrm{CH}_{3}\right), 14.8\left(\mathrm{CH}_{3}\right)$; HRMS for $\left(\mathrm{M}-\mathrm{H}^{-}\right)$, calcd: 187.13342, found: 187.13329.

These spectra correspond with the ones described in the literature. ${ }^{8}$

\section{4-carboxymethyl-5-ethylidene-6-(3,4,5-trihydroxy-6-hydroxymethyl-tetrahydro- pyran-2-yloxy)-5,6-dihydro-4H-pyran-3-carboxylic acid methyl ester or jaspolyside: ${ }^{9}$}

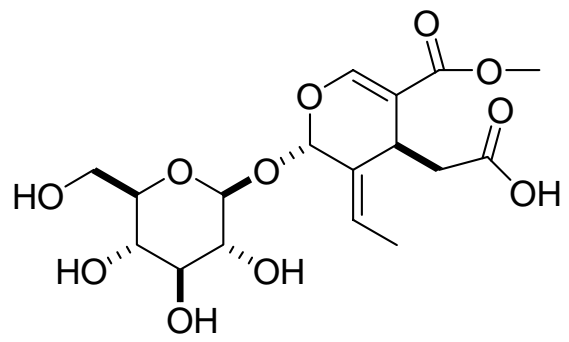

$\mathrm{C}_{17} \mathrm{H}_{24} \mathrm{O}_{11} ; \mathrm{F} . \mathrm{W} .=404.13$ g.mol ${ }^{-1}$

To a solution of oleuropein (200 $\mathrm{mg}, 0.37 \mathrm{mmol})$ in water $(2.5 \mathrm{~mL})$ under argon was directly added $7.4 \mathrm{~mL}$ of a $0.1 \mathrm{M}$ aqueous solution of $\mathrm{NaOH}$ (0.74 mmol, 2 equiv.). After $3 \mathrm{~h}$ stirring at r.t., the mixture was acidified at $0{ }^{\circ} \mathrm{C}$ with a $1 \mathrm{M}$ aqueous solution of $\mathrm{HCl}$ until $\mathrm{pH}=4-5$ was reached. The crude was then lyophilized. Purification by flash chromatography on silica gel $\left(\mathrm{CHCl}_{3} / \mathrm{MeOH}: 95 / 15 \rightarrow 5 / 5 ; \mathrm{R}_{\mathrm{f}(\mathrm{CHCl} / \mathrm{MeOH}: 8 / 2)}=0.22\right)$ gave $127 \mathrm{mg}$ of the desired carboxylic acid as a pale yellow solid (85\% yield).

\footnotetext{
8 Tanahashi, T.; Takenaka, Y.; Nagakura, N.; Nishi, T. Chem. Pharm. Bull. 2000, 48, 1200-1204.

${ }^{9}$ Capozzi, F.; Piperno, A.; Ucella, N. J. Agric. Food Chem. 2000, 48, 1623.
} 
$[\alpha]_{\mathrm{D}}{ }^{20}-45^{\circ}$ (c $\left.0.335, \mathrm{CH}_{3} \mathrm{OH}\right)$ [litt. $[\alpha]_{\mathrm{D}}{ }^{24}-53^{\circ}$ (c $\left.\left.0.075, \mathrm{CH}_{3} \mathrm{OH}\right)^{10}\right]$. IR (neat) 3346, 1695, 1629, 1438, 1305, 1160, 1072, 906, 854, 818, $769 \mathrm{~cm}^{-1} .{ }^{1} \mathrm{H}$ NMR $\left(\mathrm{D}_{2} \mathrm{O} ; 300 \mathrm{MHz}\right)$ $\delta 7.55$ (s, 1H), 6.10 (q, $J=7.0 \mathrm{~Hz}, 1 \mathrm{H}$ ), 5.93 (s, 1H), 5.25 (d, $J=7.9 \mathrm{~Hz}, 1 \mathrm{H}$ ), 3.98 (dd, $J=$ 9.6, $4.8 \mathrm{~Hz}, 1 \mathrm{H}$ ), 3.87 (dd, $J=12.5,2.1 \mathrm{~Hz}, 1 \mathrm{H}), 3.72$ (s, 3H, OMe), 3.65-3.72 (m, 1H), 3.36-3.56 (m, 4H), 2.70 (dd, $J=13.3,4.9 \mathrm{~Hz}, 1 \mathrm{H}$ ), 2.33 (dd, $J=13.3,9.5 \mathrm{~Hz}, 1 \mathrm{H}$ ), 1.71 (dd, $J=7.1,1.3 \mathrm{~Hz}, 3 \mathrm{H}) ;{ }^{13} \mathrm{C}$ NMR ( $\left.\mathrm{D}_{2} \mathrm{O} ; 75 \mathrm{MHz}\right) \delta 177.77$ (C), 169.64 (C), 154.63 (CH), $128.72(\mathrm{C}), 125.08(\mathrm{CH}), 108.97(\mathrm{C}), 99.91(\mathrm{CH}), 95.20(\mathrm{CH}), 76.71(\mathrm{CH}), 75.96$ (CH), $72.96(\mathrm{CH}), 69.77(\mathrm{CH}), 60.91\left(\mathrm{CH}_{2}\right), 52.19\left(\mathrm{CH}_{3}, \mathrm{OMe}\right), 41.63\left(\mathrm{CH}_{2}\right), 31.11$ (CH), $13.15\left(\mathrm{CH}_{3}\right)$; HRMS $\left(\mathrm{MNa}^{+}\right)$, calcd : 427.12108, found : 427.12065.

\section{4-Carboxymethyl-5-ethylidene-6-(3,4,5-triacetoxy-6-acetoxymethyl-tetrahydro- pyran-2-yloxy)-5,6-dihydro-4H-pyran-3-carboxylic acid methyl ester (15):}

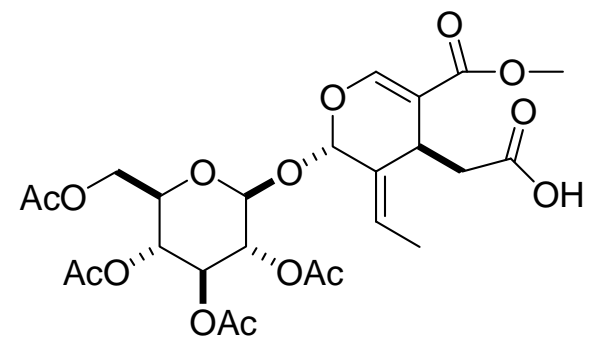

$\mathrm{C}_{25} \mathrm{H}_{32} \mathrm{O}_{15} ;$ F.W. $=572.17$ g.mol ${ }^{-1}$

To a solution of the previous oleoside $(100 \mathrm{mg}, 0.248 \mathrm{mmol})$ in pyridine $(0.8 \mathrm{~mL})$ under argon was added at $0^{\circ} \mathrm{C}$ acetic anhydride $(1.5 \mathrm{~mL}, 8.49 \mathrm{mmol}, 35$ equiv.). After $2 \mathrm{~h}$ stirring at r.t., the mixture was diluted with $\mathrm{CHCl}_{3}$, quenched with water at $0^{\circ} \mathrm{C}$, acidified with a $1 \mathrm{M}$ aqueous solution of $\mathrm{HCl}$ until $\mathrm{pH}=5$ was reached. The layers were separated; the aqueous phase was extracted with AcOEt. The combined organic layers were dried

\footnotetext{
${ }^{10}$ Shen, Y-C.; Lin, S-L.; Chein, C-C. Phytochemistry 1996, 42(6), 1629.
} 
over $\mathrm{Na}_{2} \mathrm{SO}_{4}$ and concentrated under reduced pressure. Purification by flash chromatography on silica gel $\left(\mathrm{CHCl}_{3} / \mathrm{MeOH}: 99 / 1 \rightarrow 9 / 1 ; \mathrm{R}_{\mathrm{f}(\mathrm{CHCl} / \mathrm{MeOH}: 9 / 1)}=0.56\right)$ gave 85 mg of the desired oleoside tetraacetate $\mathbf{1 5}$ as a white foam (60\% yield).

$[\alpha]_{\mathrm{D}}{ }^{20}-139.1^{\circ}$ (c $1, \mathrm{CHCl}_{3}$ ). IR (neat) 2956, 1754, 1709, 1633, 1438, 1370, 1220, 1165, 1071, 1042, 909, 856, $768 \mathrm{~cm}^{-1} .{ }^{1} \mathrm{H}$ NMR $\left(\mathrm{CHCl}_{3} ; 400 \mathrm{MHz}\right) \delta 7.43$ (s, 1H), 6.05 (q, J= $7.0 \mathrm{~Hz}, 1 \mathrm{H}), 5.66$ (s, 1H), 5.26 (t, $J=9.4 \mathrm{~Hz}, 1 \mathrm{H}), 5.08-5.14$ (m, 2H), 4.99 (d, $J=7.9 \mathrm{~Hz}$, 1H), 4.28 (dd, $J=12.4,4.6 \mathrm{~Hz}, 1 \mathrm{H}$ ), 4.13 (dd, $J=12.4,1.9 \mathrm{~Hz}, 1 \mathrm{H}$ ), 3.99 (dd, $J=8.7,4.5$ Hz, 1H), 3.74-3.77 (m, 1H), 3.72 (s, 3H, OMe), 2.77 (dd, J= 14.6, 4.4 Hz, 1H), 2.42 (dd, $J=14.6,8.8 \mathrm{~Hz}, 1 \mathrm{H}), 2.08$ (s, 3H, Ac), 2.01-2.03 (m, 9H, Ac), 1.74 (d, $J=6.9 \mathrm{~Hz}, 3 \mathrm{H}$ );

${ }^{13} \mathrm{C}$ NMR (CHCl $\left.; 100 \mathrm{MHz}\right) \delta 176.54$ (C), 171.35 (C), 170.67 (C), 169, 86 (C), 169.81 (C), 167.25 (C), 153.66 (CH), 128.24 (C), 125.55 (CH), 108.96 (C), 97.48 (CH), 94.06 (CH), $72.92(\mathrm{CH}), 72.66(\mathrm{CH}), 71.11(\mathrm{CH}), 68.62(\mathrm{CH}), 62.08\left(\mathrm{CH}_{2}\right), 51.96\left(\mathrm{CH}_{3}, \mathrm{OMe}\right)$, $40.17\left(\mathrm{CH}_{2}\right), 30.63(\mathrm{CH}), 21.18\left(\mathrm{CH}_{3}\right), 21.11\left(\mathrm{CH}_{3}\right), 21.06\left(\mathrm{CH}_{3}\right), 21.03\left(\mathrm{CH}_{3}\right), 14.08$ $\left(\mathrm{CH}_{3}\right)$; HRMS $\left(\mathrm{MH}^{+}\right)$, calcd: 573.18140, found: 573.18278; $\left(\mathrm{MNa}^{+}\right)$, calcd: 595.16334, found:595.16491. MS (ESI) m/z: $\left(\mathrm{MH}^{+}\right) 573$.

4-\{2-[2-(tert-Butyl-diphenyl-silanyloxy)-1-methyl-ethyl]-3-hydroxy-5-methylcyclopentylmethoxycarbonylmethyl\}-5-ethylidene-6-(3,4,5-triacetoxy-6acetoxymethyl-tetrahydro-pyran-2-yloxy)-5,6-dihydro-4H-pyran-3-carboxylic acid methyl ester:

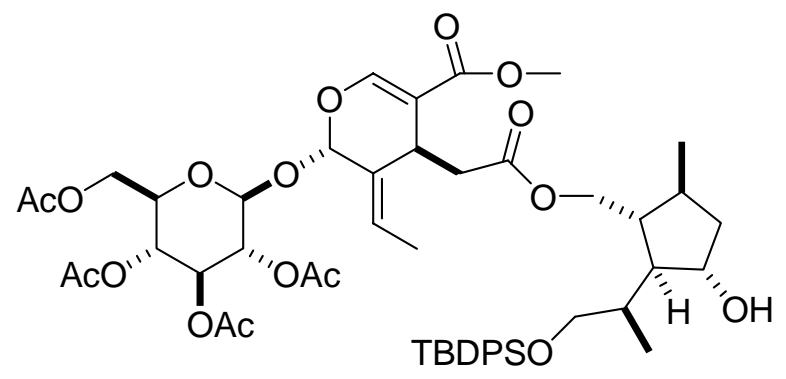

$\mathrm{C}_{51} \mathrm{H}_{68} \mathrm{O}_{17} \mathrm{Si}$; F.W. $=980.42$ g.mol $^{-1}$ 
To a solution of oleoside tetraacetate 15 (50 mg, 0.0874 mmol, 1 equiv.) in DCM (1 mL) under argon was added at $0^{\circ} \mathrm{C}$ trichlorobenzoyl chloride $(17 \mu \mathrm{L}, 0.109 \mathrm{mmol}, 1.2$ equiv.) and triethylamine (17 $\mu \mathrm{L}, 0.122 \mathrm{mmol}, 1.4$ equiv.). After $2 \mathrm{~h}$ stirring at r.t., the mixture was cooled to $0{ }^{\circ} \mathrm{C}$ and a solution of diol 12 (56 mg, $0.131 \mathrm{mmol}, 1.5$ equiv.) and DMAP (15 mg, 0.128 mmol, 1.4 equiv.) in DCM (1 mL) was added dropwise over 10 min. After stirring $2 \mathrm{~h}$ at r.t., the mixture was quenched with a saturated aqueous solution of $\mathrm{NH}_{4} \mathrm{Cl}$ and extracted with DCM and AcOEt. The combined organic layers were dried over $\mathrm{Na}_{2} \mathrm{SO}_{4}$ and concentrated under reduced pressure. Purification by flash chromatography on silica gel (hexane/AcOEt: $7 / 3 \rightarrow 5 / 5$ ) afforded $85 \mathrm{mg}$ of a mixture of the desired ester (59 mg) and of the starting alcohol 12 (26 mg) as a clear colorless oil. Further purification was carried out by preparative TLC (hexane/AcOEt: 7/3; $\mathrm{R}_{\mathrm{f}}$ ester= $0.59 ; \mathrm{R}_{\mathrm{f}}$ alcohol= 0.32 ) and gave $56 \mathrm{mg}$ of the desired ester (65\% yield) as a yellowish oil and $22 \mathrm{mg}$ of the starting alcohol 12 (69\% yield recovered).

$[\alpha]_{\mathrm{D}}^{20}-114.9^{\circ}$ (c $0.241, \mathrm{CHCl}_{3}$ ). IR (neat) 3523, 2956, 1759, 1634, 1429, 1368, 1220, 1164, 1072, 909, 802, 744, $704 \mathrm{~cm}^{-1} .{ }^{1} \mathrm{H} \mathrm{NMR}\left(\mathrm{CHCl}_{3} ; 400 \mathrm{MHz}\right) \delta$ 7.62-7.67 (m, 4H, Ar), 7.45 (s, 1H), 7.34-7.43 (m, 6H, Ar), 5.97 (q br, J= $7.2 \mathrm{~Hz}, 1 \mathrm{H}), 5.69$ (s, 1H), 5.27 (t, $J=9.5 \mathrm{~Hz}, 1 \mathrm{H}), 5.08-5.15$ (m, 2H), 5.03 (d, $J=8.0 \mathrm{~Hz}, 1 \mathrm{H}), 4.33$ (dd, $J=12.5,4.5 \mathrm{~Hz}$, 1H), 4.17 (dd, $J=10.9,4.2 \mathrm{~Hz}, 1 \mathrm{H}), 4.10$ (dd, $J=12.5,2.3 \mathrm{~Hz}, 1 \mathrm{H}), 4.06$ (q, $J=4.5 \mathrm{~Hz}$, 1H), 3.98 (dd, $J=8.7,3.9 \mathrm{~Hz}, 1 \mathrm{H}), 3.83$ (dd, $J=11.0,6.6 \mathrm{~Hz}, 1 \mathrm{H}$ ), 3.75 (dq, $J=10.0,2.4$ Hz, 1H), 3.70 (s, 3H, OMe), 3.58 (dq, $J=10.5,5.7 \mathrm{~Hz}, 2 \mathrm{H}$ ), 2.70 (dd, $J=14.5,4.2 \mathrm{~Hz}$, 1H), 2.37 (dd, $J=14.5,9.0 \mathrm{~Hz}, 1 \mathrm{H}$ ), 2.06 (s, 3H, Ac), 2.03 (s, 3H, Ac), 2.02 (s, 3H, Ac), 2.02 (s, 3H, Ac), 1.71 (dd, J= 7.1, $1.3 \mathrm{~Hz}, 3 \mathrm{H}), 1.73-1.78$ (m, 1H), 1.60-1.68 (m, 1H), 
1.37-1.59 (m, 4H), 1.04 (s, 9H), 0.97 (d, $J=6.8 \mathrm{~Hz}, 3 \mathrm{H}), 0.91(\mathrm{~s}, J=6.8 \mathrm{~Hz}, 3 \mathrm{H}) ;{ }^{13} \mathrm{C}$ NMR ( $\left.\mathrm{CHCl}_{3} ; 75 \mathrm{MHz}\right) \delta 171.34$ (C), 170.75 (C), 170.32 (C), 169.54 (C), 169.50 (C), $166.88(\mathrm{C}), 153.11(\mathrm{CH}), 135.75(2 * \mathrm{CH}), 135.71(2 * \mathrm{CH}), 133.50$ (C), 133.46 (C), 129.87 (CH), $128.20(\mathrm{C}), 127.84(4 * \mathrm{CH}), 124.91(\mathrm{CH}), 108.83(\mathrm{C}), 97.03(\mathrm{CH}), 93.65(\mathrm{CH})$, $75.75(\mathrm{CH}), 72.61(\mathrm{CH}), 72.34(\mathrm{CH}), 70.79(\mathrm{CH}), 68.24(\mathrm{CH}), 68.04\left(\mathrm{CH}_{2}\right), 67.25\left(\mathrm{CH}_{2}\right)$, $61.75\left(\mathrm{CH}_{2}\right), 54.16(\mathrm{CH}), 51.62\left(\mathrm{CH}_{3}, \mathrm{OMe}\right), 48.47(\mathrm{CH}), 42.73\left(\mathrm{CH}_{2}\right), 40.19\left(\mathrm{CH}_{2}\right)$, $38.96(\mathrm{CH}), 34.60(\mathrm{CH}), 30.42(\mathrm{CH}), 29.82(\mathrm{CH}), 26.97\left(3^{*} \mathrm{CH}_{3}\right), 20.84\left(\mathrm{CH}_{3}, \mathrm{Ac}\right), 20.80$ $\left(\mathrm{CH}_{3}, \mathrm{Ac}\right), 20.75\left(\mathrm{CH}_{3}, \mathrm{Ac}\right), 20.72\left(\mathrm{CH}_{3}, \mathrm{Ac}\right), 20.41\left(\mathrm{CH}_{3}\right), 19.32(\mathrm{C}), 15.74\left(\mathrm{CH}_{3}\right)$, $13.73\left(\mathrm{CH}_{3}\right)$; HRMS $\left(\mathrm{MH}^{+}\right)$, calcd: 981.42985, found: 981.42945; $\left(\mathrm{MNa}^{+}\right)$, calcd: 1003.41180, found: 1003.41110. MS (ESI) m/z: $\left(\mathrm{MH}^{+}\right) 981$.

5-Ethylidene-4-[3-hydroxy-2-(2-hydroxy-1-methyl-ethyl)-5-methylcyclopentylmethoxycarbonylmethyl]-6-(3,4,5-triacetoxy-6-acetoxymethyltetrahydro-pyran-2-yloxy)-5,6-dihydro-4H-pyran-3-carboxylic acid methyl ester:

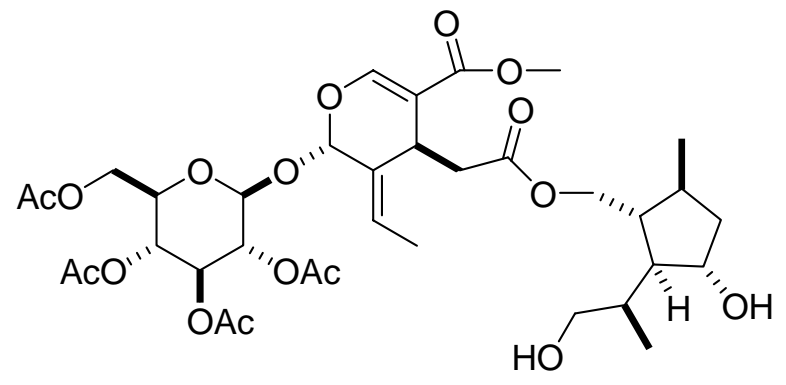

$\mathrm{C}_{35} \mathrm{H}_{50} \mathrm{O}_{17} ; \mathrm{F} . \mathrm{W} .=742.30$ g. $\mathrm{mol}^{-1}$

To a solution of the previous ester $(27.5 \mathrm{mg}, 0.028 \mathrm{mmol})$ in THF $(3 \mathrm{~mL})$ at $0{ }^{\circ} \mathrm{C}$ under argon was added $30 \mu \mathrm{L}$ of a $1 \mathrm{M}$ solution of TBAF in THF (1 equiv.) After stirring 30 min at r.t., the mixture was quenched with a saturated aqueous solution of $\mathrm{NH}_{4} \mathrm{Cl}$ and extracted with AcOEt. The combined organic layers were dried over $\mathrm{Na}_{2} \mathrm{SO}_{4}$ and concentrated under reduced pressure. Purification by flash chromatography on silica gel 


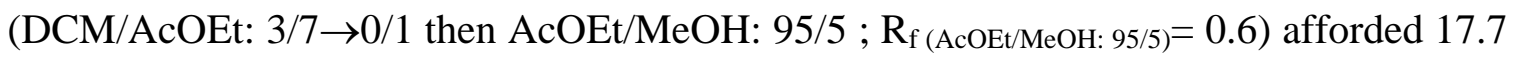
mg of the deprotected alcohol (85\% yield) as a clear viscous oil. $[\alpha]_{\mathrm{D}}{ }^{20}-60.6^{\circ}$ (c 0.246, $\mathrm{CHCl}_{3}$ ). IR (neat) 3436 (broad), 2928, 1754, 1633, 1439, 1376, 1227, 1165, 1042, $800 \mathrm{~cm}^{-1} .{ }^{1} \mathrm{H}$ NMR $\left(\mathrm{CHCl}_{3} ; 400 \mathrm{MHz}\right) \delta 7.48$ (s, 1H), 6.03 (q br, $J=$ $6.7 \mathrm{~Hz}, 1 \mathrm{H}$ ), 5.71 (s, 1H), 5.28 (t, $J=9.4 \mathrm{~Hz}, 1 \mathrm{H}), 5.12-5.19$ (m, 2H), 5.06 (d, $J=8.0 \mathrm{~Hz}$, 1H), 4.34 (dd, $J=12.4,4.5 \mathrm{~Hz}, 1 \mathrm{H}$ ), 4.12-4.18 (m, 3H), 4.02 (q, $J=4.1 \mathrm{~Hz}, 1 \mathrm{H}$ ), 3.97 (dd, $J=10.9,6.0 \mathrm{~Hz}, 1 \mathrm{H}), 3.78$ (dq, $J=10.0,2.3 \mathrm{~Hz}, 1 \mathrm{H}$ ), 3.75 (s, 3H, OMe), 3.67 (dd, $J=$ 11.1, $5.0 \mathrm{~Hz}, 1 \mathrm{H}$ ), 3.56 (dd, $J=11.0,6.0 \mathrm{~Hz}, 1 \mathrm{H}), 2.75$ (dd, $J=14.5,4.2 \mathrm{~Hz}, 1 \mathrm{H}$ ), 2.42 (dd, J= 14.5, $9.0 \mathrm{~Hz}, 1 \mathrm{H}$ ), 2.12 (s, 3H, Ac), 2.06 (s, 3H, Ac), 2.06 (s, 3H, Ac), 2.05 (s, 3H, Ac), 1.77-1.85 (m, 3H), 1.76 (dd, J= 7.0, $1.2 \mathrm{~Hz}, 3 \mathrm{H}$ ), 1.51-1.67 (m, 3H), 1.01 (dd, $J=6.9 \mathrm{~Hz}, 3 \mathrm{H}), 0.96(\mathrm{dd}, \quad J=6.9 \mathrm{~Hz}, 3 \mathrm{H}) ;{ }^{13} \mathrm{C} \mathrm{NMR}\left(\mathrm{CHCl}_{3} ; 100 \mathrm{MHz}\right)$ $\delta 171.47$ (C), 170.94 (C), 170.35 (C), 169.57 (C), 169.54 (C), 167.06 (C), 153.26 (CH), 128.16 (C), 124.97 (CH), 108.82 (C), $97.05(\mathrm{CH}), 93.61(\mathrm{CH}), 76.63(\mathrm{CH}), 72.60(\mathrm{CH})$, $72.34(\mathrm{CH}), 70.79(\mathrm{CH}), 68.32(\mathrm{CH}), 67.81\left(\mathrm{CH}_{2}\right), 67.09\left(\mathrm{CH}_{2}\right), 61.87\left(\mathrm{CH}_{2}\right), 53.52$ (CH), $51.72\left(\mathrm{CH}_{3}, \mathrm{OMe}\right), 48.11(\mathrm{CH}), 42.60\left(\mathrm{CH}_{2}\right), 40.28\left(\mathrm{CH}_{2}\right), 39.27(\mathrm{CH}), 34.73$ (CH), $30.50(\mathrm{CH}), 20.91\left(\mathrm{CH}_{3}, \mathrm{Ac}\right), 20.83\left(\mathrm{CH}_{3}, \mathrm{Ac}\right), 20.77\left(\mathrm{CH}_{3}, \mathrm{Ac}\right), 20.75\left(\mathrm{CH}_{3}, \mathrm{Ac}\right)$, $20.68\left(\mathrm{CH}_{3}\right), 15.45\left(\mathrm{CH}_{3}\right), 13.77\left(\mathrm{CH}_{3}\right)$; HRMS $\left(\mathrm{MH}^{+}\right)$, calcd: 743.31208 , found: 743.31136; $\left(\mathrm{MNa}^{+}\right)$, calcd: 765.29402, found: 765.29335. MS (ESI) m/z: $\left(\mathrm{MH}^{+}\right) 743$. 
5-Ethylidene-4-[3-hydroxy-2-(2-hydroxy-1-methyl-ethyl)-5-methylcyclopentylmethoxycarbonylmethyl]-6-(3,4,5-trihydroxy-6-hydroxymethyltetrahydro-pyran-2-yloxy)-5,6-dihydro-4H-pyran-3-carboxylic acid methyl ester or Nudifloside D (1):

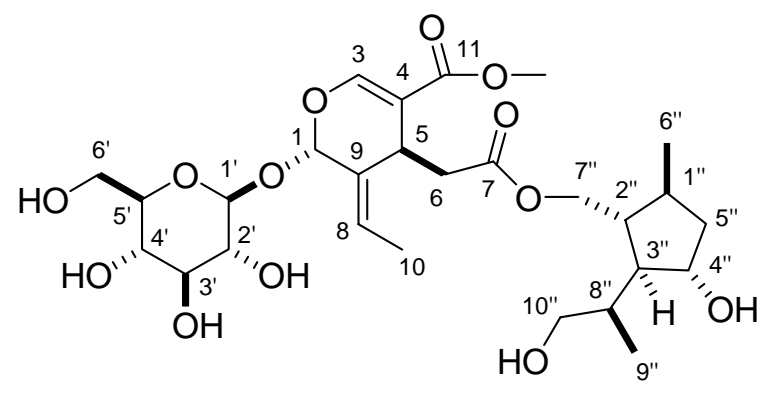

$\mathrm{C}_{27} \mathrm{H}_{42} \mathrm{O}_{13} ; \mathrm{F} . \mathrm{W} .=574.26$ g.mol ${ }^{-1}$

To a solution of the previous deprotected ester (17.7 $\mathrm{mg}, 0.0238 \mathrm{mmol})$ in $\mathrm{MeOH}$ (0.5 mL) at $0{ }^{\circ} \mathrm{C}$ under argon was added diethylamine (12 $\mu \mathrm{L}, 0.119$ mmol, 5.0 equiv.). After stirring $9 \mathrm{~h}$ at r.t., the mixture was diluted with $\mathrm{MeOH}$ and quenched with a small amount of silica gel. After evaporation of the solvents under reduced pressure, the crude was directly submitted to flash chromatography on silica gel (AcOEt/MeOH: $1 / 0 \rightarrow 9 / 1$; $\mathrm{R}_{\mathrm{f}(\mathrm{AcOEt} / \mathrm{MeOH}: 9 / 1)}=0.2$ ) to afford $7.65 \mathrm{mg}$ of Nudifloside $\mathbf{D}, \mathbf{1}$ as a clear viscous oil. Redilution in water followed by lyophilization gave $7.6 \mathrm{mg}$ of a white powder (56\% yield).

$[\alpha]_{\mathrm{D}}{ }^{24}-135^{\circ}\left(\mathrm{c} 0.5, \mathrm{CH}_{3} \mathrm{OH}\right)$ [litt. $[\alpha]_{\mathrm{D}}{ }^{24}-161^{\circ}$ (c $\left.\left.0.41, \mathrm{CH}_{3} \mathrm{OH}^{11}\right)\right]$. IR (neat) 3370 (broad), 2919, 1709, 1631, 1439, 1383, 1304, 1160, 1076, $1044 \mathrm{~cm}^{-1} . \mathrm{HRMS}\left(\mathrm{MH}^{+}\right)$, calcd: 575.26982, found: 575.26987 $\left(\mathrm{MNa}^{+}\right)$, calcd: 597.25176, found: 597.25152. LC/MS (ESI) m/z: Rt= 3.96 min, $\left(\mathrm{MH}^{+}\right)$575, $\left(\mathrm{MNa}^{+}\right) 597$.

\footnotetext{
${ }^{11}$ Takenaka, Y.; Tanahashi, T.; Tagueshi, H.; Nagakura, N.; Nishi, T. Chem. Pharm. Bull. 2002, 50, 3, 384.
} 


\begin{tabular}{|c|c|c|}
\hline $\begin{array}{c}\text { Hor } \\
\text { C }\end{array}$ & $\begin{array}{c}{ }^{1} \mathrm{H} \text { NMR } \\
(\mathrm{MeOD}, 500 \mathrm{MHz}) \delta\end{array}$ & $\begin{array}{c}{ }^{13} \mathrm{C} \text { NMR } \\
(\mathrm{MeOD}, 125 \mathrm{MHz}) \delta\end{array}$ \\
\hline 1 & $5.96(\mathrm{~s} \mathrm{br}, 1 \mathrm{H})$ & 95.0 \\
\hline 3 & $7.55(\mathrm{~s}, 1 \mathrm{H})$ & 155.1 \\
\hline 4 & - & 109.4 \\
\hline 5 & $4.04(\mathrm{dd}, J=9.2,4.5 \mathrm{~Hz}, 1 \mathrm{H})$ & 31.9 \\
\hline 6 & $\begin{array}{l}2.75(\mathrm{dd}, J=14.2,4.5 \mathrm{~Hz}, 1 \mathrm{H}) \\
2.51(\mathrm{dd}, J=14.2,9.2 \mathrm{~Hz}, 1 \mathrm{H})\end{array}$ & 41.3 \\
\hline 7 & - & 173.3 \\
\hline 8 & $6.14(\mathrm{q} \mathrm{br}, J=7.2 \mathrm{~Hz}, 1 \mathrm{H})$ & 124.8 \\
\hline 9 & - & 130.7 \\
\hline 10 & $1.77(\mathrm{dd}, J=7.1,1.4 \mathrm{~Hz}, 3 \mathrm{H})$ & 13.7 \\
\hline 11 & - & 168.6 \\
\hline OMe & $3.74(\mathrm{~s}, 3 \mathrm{H})$ & 51.9 \\
\hline 1 & $4.83(\mathrm{~d}, J=7.8 \mathrm{~Hz}, 1 \mathrm{H})$ & 100.7 \\
\hline $2^{\prime}$ & $3.31-3.38(\mathrm{~m}, 1 \mathrm{H})$ & 74.8 \\
\hline $3^{\prime}$ & $3.43(\mathrm{t}, J=9.2 \mathrm{~Hz}, 1 \mathrm{H})$ & 78.0 \\
\hline 4 & $3.31-3.38(\mathrm{~m}, 1 \mathrm{H})$ & 71.5 \\
\hline 5 & $3.31-3.38(\mathrm{~m}, 1 \mathrm{H})$ & 78.5 \\
\hline 6' & $\begin{array}{l}3.91(\mathrm{dd}, J=12.1,2.0 \mathrm{~Hz}, 1 \mathrm{H}) \\
3.69(\mathrm{dd}, J=12.0,5.6 \mathrm{~Hz}, 1 \mathrm{H})\end{array}$ & 62.7 \\
\hline 1" & $2.04(\mathrm{~m}, 1 \mathrm{H})$ & 36.5 \\
\hline 2” & $1.61(\mathrm{~m}, 1 \mathrm{H})$ & 48.5 \\
\hline 3” & $1.61(\mathrm{~m}, 1 \mathrm{H})$ & 54.8 \\
\hline 4” & $4.07(\mathrm{~m}, 1 \mathrm{H})$ & 76.4 \\
\hline $5 ”$ & $\begin{array}{c}1.80(\mathrm{~m}, 1 \mathrm{H}) \\
1.46(\mathrm{ddd}, J=14.6,9.0,5.6 \mathrm{~Hz}, 1 \mathrm{H})\end{array}$ & 43.9 \\
\hline 6” & $1.06(\mathrm{~d}, J=6.8 \mathrm{~Hz}, 3 \mathrm{H})$ & 20.5 \\
\hline 7” & $\begin{array}{l}4.19(\mathrm{dd}, J=10.9,4.5 \mathrm{~Hz}, 1 \mathrm{H}) \\
3.96(\mathrm{dd}, J=10.9,6.5 \mathrm{~Hz}, 1 \mathrm{H})\end{array}$ & 69.4 \\
\hline 8” & $1.75(\mathrm{~m}, 1 \mathrm{H})$ & 39.3 \\
\hline 9” & $0.99(\mathrm{~d}, J=6.9 \mathrm{~Hz}, 3 \mathrm{H})$ & 15.2 \\
\hline $10 ”$ & $\begin{array}{l}3.59(\mathrm{dd}, J=10.8,5.6 \mathrm{~Hz}, 1 \mathrm{H}) \\
3.43(\mathrm{dd}, J=10.8,7.3 \mathrm{~Hz}, 1 \mathrm{H})\end{array}$ & 67.0 \\
\hline
\end{tabular}

Significant COSY correlations: H-1" $\Leftrightarrow \mathrm{H}_{2}-5 ”$; H-1" $\Leftrightarrow \mathrm{H}_{3}-6 ”$; H-2" $\Leftrightarrow \mathrm{H}-3$ ” ; H-2” $\Leftrightarrow \mathrm{H}-$ 7" ; H-3” $\Leftrightarrow H-4 ” ; H-4 ” \Leftrightarrow \mathrm{H}_{2}-5 ” ; H-8 ” \Leftrightarrow \mathrm{H}_{3}-9 ” ; \mathrm{H}-8 ” \Leftrightarrow \mathrm{H}_{2}-10 ”$; Significant HMBC

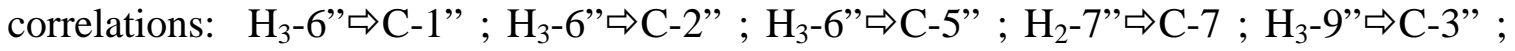
$\mathrm{H}_{3}-9 ” \Rightarrow \mathrm{C}-8 ” ; \mathrm{H}_{2}-6 \Rightarrow \mathrm{C}-7$; OMe $\Rightarrow \mathrm{C}-11$. 
These spectra correspond with the ones described in the literature. ${ }^{10}$

4-\{2-[2-(tert-Butyl-diphenyl-silanyloxy)-1-methyl-ethyl]-3-hydroxycarbonylmethyl5-methyl-cyclopentylmethoxycarbonylmethyl\}-Bis-[5-ethylidene-6-(3,4,5-triacetoxy6-acetoxymethyl-tetrahydro-pyran-2-yloxy)-5,6-dihydro-4H-pyran-3-carboxylic acid methyl ester]:

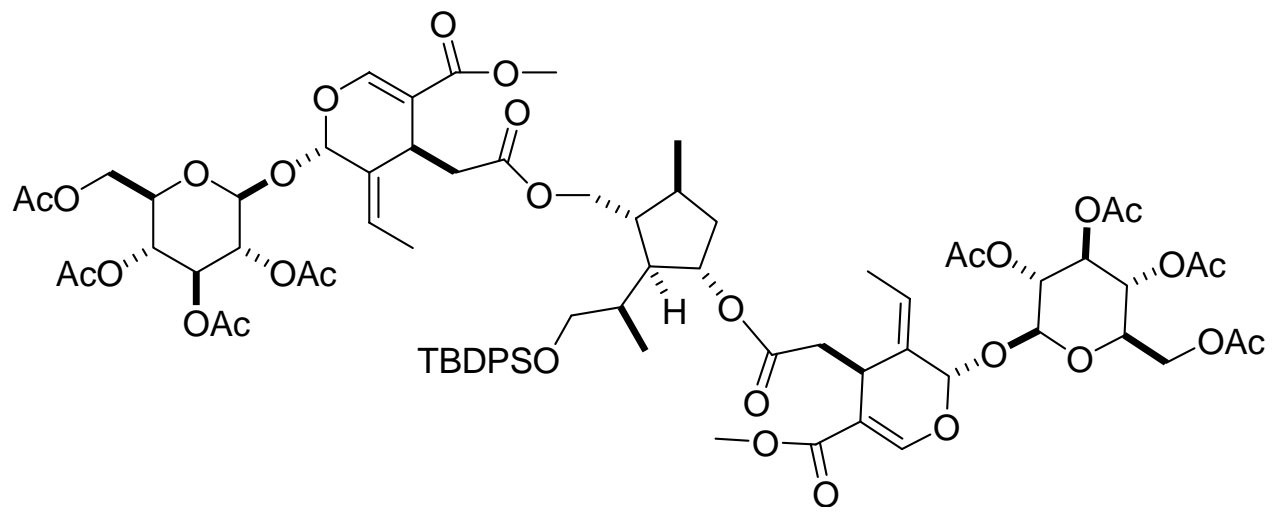

$\mathrm{C}_{76} \mathrm{H}_{98} \mathrm{O}_{31} \mathrm{Si}$; F.W. $=1534.59$ g.mol ${ }^{-1}$

To a solution of oleoside tetraacetate ester 15 (60 mg, $0.104 \mathrm{mmol}, 3.6$ equiv.) in DCM $(1 \mathrm{~mL})$ under argon was added at $0^{\circ} \mathrm{C}$ trichlorobenzoyl chloride $(20 \mu \mathrm{L}, 0.128$ mmol, 4.5 equiv.) and triethylamine (20 $\mu \mathrm{L}, 0.145 \mathrm{mmol}, 5.1$ equiv.). After $2 \mathrm{~h}$ stirring at r.t., the mixture was cooled to $0{ }^{\circ} \mathrm{C}$ and a solution of the ester $12(28 \mathrm{mg}, 0.0286 \mathrm{mmol}, 1$ equiv.) and DMAP (18 mg, $0.148 \mathrm{mmol}, 5.2$ equiv.) in DCM (1 mL) was added dropwise over $10 \mathrm{~min}$. After stirring $2 \mathrm{~h}$ at r.t., the mixture was quenched with a $1 \mathrm{M}$ aqueous solution of $\mathrm{HCl}$ at $0^{\circ} \mathrm{C}$ and extracted with DCM and AcOEt. The combined organic layers were dried over $\mathrm{Na}_{2} \mathrm{SO}_{4}$ and concentrated under reduced pressure. Purification by flash chromatography on silica gel (hexane/AcOEt: 7/3 $\rightarrow 5 / 5$; $\mathrm{R}_{\mathrm{f}(\text { hexane/AcOEt: } 4 / 6)}=0.57$ ) afforded $41.7 \mathrm{mg}$ of a the desired di-ester (95\% yield) as a yellowish oil.

$[\alpha]_{\mathrm{D}}{ }^{20}-91.1^{\circ}$ (c 1.043, $\mathrm{CHCl}_{3}$ ). IR (neat) 2954, 1757, 1710, 1632, 1439, 1370, 1221, 1071, 1043, $703 \mathrm{~cm}^{-1} .{ }^{1} \mathrm{H}$ NMR $\left(\mathrm{CHCl}_{3} ; 400 \mathrm{MHz}\right) \delta$ 7.62-7.80 (m, 4H, Ar), 7.48 (s, 1H), 
7.46 (s, 1H), 7.36-7.45 (m, 6H, Ar), 6.01 (q br, $J=7.2 \mathrm{~Hz}, 1 \mathrm{H}$ ), 5.99 (q br, $J=7.2 \mathrm{~Hz}$, 1H), 5.73 (s br, 2H), 5.30 (t, $J=9.4 \mathrm{~Hz}, 2 \mathrm{H}$ ), 5.11-5.18 (m, 4H), 5.60 (d, $J=8.1 \mathrm{~Hz}, 1 \mathrm{H}$ ), 5.44 (d, $J=8.0 \mathrm{~Hz}, 1 \mathrm{H}), 4.34$ (dd, $J=12.4,4.3 \mathrm{~Hz}, 2 \mathrm{H}), 4.17$ (dd, $J=10.9,5.1 \mathrm{~Hz}, 2 \mathrm{H}$ ), 4.11 (dq, $J=10.5,2.4 \mathrm{~Hz}, 2 \mathrm{H}), 3.88-4.02$ (m, 3H), 3.75-3.80 (m, 2H), 3.72 (s, 3H, OMe), 3.71 (s, 3H, OMe), 3.62 (dd, $J=9.9,5.3 \mathrm{~Hz}, 1 \mathrm{H}$ ), 3.48 (dd, $J=9.9,5.3 \mathrm{~Hz}, 1 \mathrm{H}$ ), 2.69 (dd, $J=15.0,4.2 \mathrm{~Hz}, 1 \mathrm{H}$ ), 2.62 (dd, $J=14.7,4.7 \mathrm{~Hz}, 1 \mathrm{H}), 2.39$ (dd, $J=14.8,9.2 \mathrm{~Hz}, 1 \mathrm{H}$ ), 2.37 (J= 14.8, $9.2 \mathrm{~Hz}, 1 \mathrm{H}$ ), 2.08 (s, 6H, Ac), 2.07 (s, 6H, Ac), 2.06 (s, 6H, Ac), 2.03 (s, 6H, Ac), 1.76-1.85 (m, 3H) , 1.73 (d, $J=6.9 \mathrm{~Hz}, 6 \mathrm{H}), 1.54-1.62(\mathrm{~m}, 1 \mathrm{H}), 1.35-1.45$ (m, 2H), 1.05 (s, 9H), 1.00 (d, $J=6.5 \mathrm{~Hz}, 3 \mathrm{H}), 0.92$ (d, $J=6.2 \mathrm{~Hz}, 3 \mathrm{H}) ;{ }^{13} \mathrm{C} \mathrm{NMR}\left(\mathrm{CHCl}_{3} ; 100\right.$ MHz) $\delta 171.25$ (C), $170.83(\mathrm{C}), 170.81(\mathrm{C}), 170.46(2 * \mathrm{C}), 169.63(2 * \mathrm{C}), 169.58\left(2{ }^{*} \mathrm{C}\right)$, $166.92(2 * \mathrm{C}), 165.93(\mathrm{C}), 153.18(\mathrm{CH}), 153.09(\mathrm{CH}), 136.48(\mathrm{CH}), 135.75(2 * \mathrm{CH})$, $135.71(2 * \mathrm{CH}), 133.69(2 * \mathrm{C}), 132.65(\mathrm{CH}), 131.73(\mathrm{CH}), 129.81(\mathrm{CH}), 128.58(\mathrm{C})$, $128.27(\mathrm{CH}+\mathrm{C}), 127.83(4 * \mathrm{CH}), 125.03(\mathrm{CH}), 124.85(\mathrm{CH}), 108.90(\mathrm{C}), 108.77(\mathrm{C})$, $97.23(\mathrm{CH}), 97.16(\mathrm{CH}), 94.07(\mathrm{CH}), 93.84(\mathrm{CH}), 78.83(\mathrm{CH}), 72.67(2 * \mathrm{CH}), 72.31$ (CH), $70.85(\mathrm{CH}), 68.28\left(\mathrm{CH}_{2}\right), 67.89\left(\mathrm{CH}_{2}\right), 66.85\left(\mathrm{CH}_{2}\right), 61.79\left(\mathrm{CH}_{2}\right), 53.58(\mathrm{CH})$, $51.60\left(2 * \mathrm{CH}_{3}, \mathrm{OMe}\right), 47.05(\mathrm{CH}), 41.01\left(\mathrm{CH}_{2}\right), 40.39\left(\mathrm{CH}_{2}\right), 40.16\left(\mathrm{CH}_{2}\right), 37.43(\mathrm{CH})$, $36.53(\mathrm{CH}), 30.27(\mathrm{CH}), 30.16(\mathrm{CH}), 29.85(\mathrm{CH}), 26.97\left(3 * \mathrm{CH}_{3}\right), 20.85\left(2 * \mathrm{CH}_{3}, \mathrm{Ac}\right)$, $20.83\left(2 * \mathrm{CH}_{3}, \mathrm{Ac}\right), 20.78\left(2 * \mathrm{CH}_{3}, \mathrm{Ac}\right), 20.75\left(2 * \mathrm{CH}_{3}, \mathrm{Ac}\right), 19.59\left(\mathrm{CH}_{3}\right), 19.32(\mathrm{C})$, $15.16\left(\mathrm{CH}_{3}\right), 13.79\left(\mathrm{CH}_{3}\right), 13.72\left(\mathrm{CH}_{3}\right)$; HRMS $\left(\mathrm{MNa}^{+}\right)$, calcd: 1557.57535, found: 1557.57322. MS (ESI) m/z: $\left(\mathrm{MNa}^{+}\right) 1557$. 


\section{4-[3-Hydroxycarbonylmethyl-2-(2-hydroxy-1-methyl-ethyl)-5-methyl-}

cyclopentylmethoxycarbonylmethyl]-Bis-[5-ethylidene-6-(3,4,5-triacetoxy-6acetoxymethyl-tetrahydro-pyran-2-yloxy)-5,6-dihydro-4H-pyran-3-carboxylic acid methyl ester]:

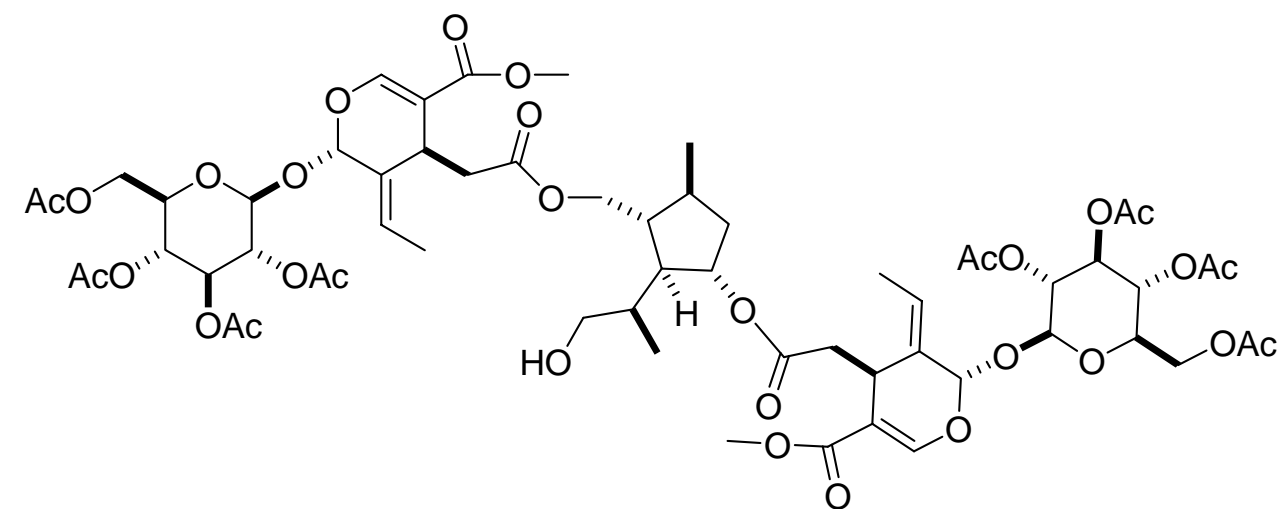

$\mathrm{C}_{60} \mathrm{H}_{80} \mathrm{O}_{31}$; F.W. = 1296.87 g.mol ${ }^{-1}$

To a solution of the previous di-ester (41.7 mg, $0.0272 \mathrm{mmol}, 1$ equiv.) in THF (3.5 mL) at $0{ }^{\circ} \mathrm{C}$ under argon was added $48 \mu \mathrm{L}$ of a $1 \mathrm{M}$ solution of TBAF in THF (1.5 equiv.) After stirring overnight at r.t., the mixture was quenched with a saturated aqueous solution of $\mathrm{NH}_{4} \mathrm{Cl}$ and extracted with AcOEt. The combined organic layers were dried over $\mathrm{Na}_{2} \mathrm{SO}_{4}$ and concentrated under reduced pressure. Purification by flash chromatography on silica gel (hexane/AcOEt: $7 / 3 \rightarrow 2 / 8$; $\mathrm{R}_{\mathrm{f}(\text { hexane/AcOEt: } 2 / 8)}=0.5$ ) afforded $26 \mathrm{mg}$ of the deprotected alcohol (74\% yield) as a white solid and $10 \mathrm{mg}$ of starting material (24) (conversion rate: 76\%).

$[\alpha]_{\mathrm{D}}{ }^{20}-105.0^{\circ}$ (c $0.767, \mathrm{CHCl}_{3}$ ). IR (neat) 3518 (broad), 2957, 1756, 1709, 1633, 1437,

1369, 1303, 1222, 1164, 1070, 1041, 908, $756 \mathrm{~cm}^{-1} .{ }^{1} \mathrm{H}$ NMR $\left(\mathrm{CHCl}_{3} ; 400 \mathrm{MHz}\right) \delta 7.48$ (s, 1H), 7.47 (s, 1H), 6.04 (q br, $J=6.8 \mathrm{~Hz}, 1 \mathrm{H}), 6.02$ (q br, $J=6.8 \mathrm{~Hz}, 1 \mathrm{H}), 5.73$ (s br, 1H), 5.71 (s br, 1H), 5.29 (t, J=9.4 Hz, 2H), 5.10-5.33 (m, 4H), 5.07 (d, J=8.0 Hz, 1H), 5.06 (d, $J=8.0 \mathrm{~Hz}, 1 \mathrm{H}), 4.34$ (dd, $J=11.7,4.2 \mathrm{~Hz}, 1 \mathrm{H}), 4.34$ (dd, $J=11.7,4.2 \mathrm{~Hz}, 1 \mathrm{H})$, 4.08-4.16 (m, 4H), 3.92-4.04 (m, 3H), 3.76-3.82 (m, 2H), 3.74 (s, 6H, OMe), 3.69 (dd, J= 
11.2, $4.7 \mathrm{~Hz}, 1 \mathrm{H}$ ), 3.51 (dd, $J=11.2,5.2 \mathrm{~Hz}, 1 \mathrm{H}$ ), 2.74 (dd, $J=14.8,4.4 \mathrm{~Hz}, 1 \mathrm{H}$ ), 2.65 (dd, $J=14.0,5.1 \mathrm{~Hz}, 1 \mathrm{H}$ ), 2.38-2.50 (m, 2H), 2.11 (s, 6H, Ac), 2.05 (s, 6H, Ac), 2.04 (s, 6H, Ac), 2.04 (s, 6H, Ac), 1.78-1.90 (m, 2H), 1.77 (d, $J=7.0 \mathrm{~Hz}, 6 \mathrm{H}), 1.57-1.70$ (m, 3H), 1.42-1.52 (m, 1H), 1.06 (d, $J=6.5 \mathrm{~Hz}, 3 \mathrm{H}), 0.96$ (d, $J=6.8 \mathrm{~Hz}, 3 \mathrm{H}) ;{ }^{13} \mathrm{C} \mathrm{NMR}\left(\mathrm{CHCl}_{3}\right.$; $100 \mathrm{MHz}) \delta 171.54(\mathrm{C}), 171.20(\mathrm{C}), 170.79(2 * \mathrm{C}), 170.35(2 * \mathrm{C}), 169.56(2 * \mathrm{C}), 169.51$ (C), 169.50 (C), 166.98 (C), 166.94 (C), 153.23 (2*CH), 128.47 (C), 128.18 (C), 125.02 (CH), $124.80(\mathrm{CH}), 108.75$ (C), $108.73(\mathrm{C}), 97.20(\mathrm{CH}), 97.14(\mathrm{CH}), 93.86(\mathrm{CH}), 93.76$ (CH), $79.91(\mathrm{CH}), 72.63(2 * \mathrm{CH}), 72.31(\mathrm{CH}), 70.82(\mathrm{CH}), 68.63(\mathrm{CH}), 68.38(\mathrm{CH})$, $68.29\left(\mathrm{CH}_{2}\right), 65.95\left(\mathrm{CH}_{2}\right), 61.92\left(\mathrm{CH}_{2}\right), 61.80\left(\mathrm{CH}_{2}\right), 51.68\left(\mathrm{CH}_{3}, \mathrm{OMe}\right), 51.63\left(\mathrm{CH}_{3}\right.$, OMe), $50.98(\mathrm{CH}), 47.46(\mathrm{CH}), 40.58\left(\mathrm{CH}_{2}\right), 40.34\left(\mathrm{CH}_{2}\right), 40.21\left(\mathrm{CH}_{2}\right), 38.33(\mathrm{CH})$, $36.85(\mathrm{CH}), 30.45(\mathrm{CH}), 30.33(\mathrm{CH}), 29.84(\mathrm{CH}), 20.88\left(2 * \mathrm{CH}_{3}, \mathrm{Ac}\right), 20.82\left(2 * \mathrm{CH}_{3}\right.$, Ac), $20.75\left(4 * \mathrm{CH}_{3}, \mathrm{Ac}\right), 19.95\left(\mathrm{CH}_{3}\right), 14.77\left(\mathrm{CH}_{3}\right), 13.77\left(\mathrm{CH}_{3}\right), 13.75\left(2 * \mathrm{CH}_{3}\right)$; HRMS (MNa ${ }^{+}$), calcd: 1319.45758, found: 1319.45612. MS (ESI) m/z: $\left(\mathrm{MH}^{+}\right) 1297$.

4-[3-Hydroxycarbonylmethyl-2-(2-hydroxy-1-methyl-ethyl)-5-methylcyclopentylmethoxycarbonylmethyl]-Bis-[5-ethylidene-6-(3,4,5-trihydroxy-6hydroxymethyl-tetrahydro-pyran-2-yloxy)-5,6-dihydro-4H-pyran-3-carboxylic acid methyl ester] or Nudifloside A (2):

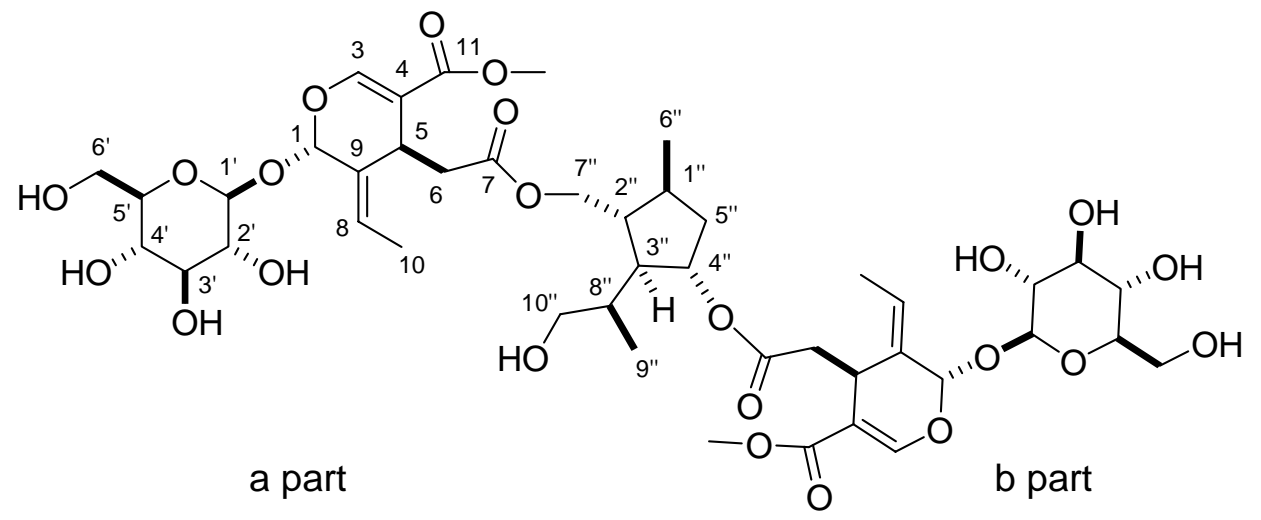

$\mathrm{C}_{44} \mathrm{H}_{63} \mathrm{O}_{23} ;$ F.W. $=960.38$ g.mol $^{-1}$ 
To a solution of the previous deprotected di-ester (26 $\mathrm{mg}, 0.0201 \mathrm{mmol})$ in methanol $(0.5 \mathrm{~mL})$ at $0^{\circ} \mathrm{C}$ under argon was added diethylamine $(21 \mu \mathrm{L}, 0.201 \mathrm{mmol}, 10$ equiv.). After stirring $9 \mathrm{~h}$ at r.t. the crude was directly submitted to flash chromatography on silica gel (AcOEt/MeOH: $\left.1 / 0 \rightarrow 9 / 1 ; \mathrm{R}_{\mathrm{f}}(\mathrm{CHCl} / \mathrm{MeOH}: 7 / 3)=0.61\right)$ to afford a colorless viscous oil which was redissolved in water and lyophilized to give $10 \mathrm{mg}$ of Nudifloside A, 2 (53\% yield) as a colorless amorphous powder.

$[\alpha]_{\mathrm{D}}{ }^{24}-200^{\circ}$ (c $\left.0.5, \mathrm{CH}_{3} \mathrm{OH}\right)$ [litt. $[\alpha]_{\mathrm{D}}{ }^{24}-203^{\circ}$ (c $\left.\left.1.27, \mathrm{CH}_{3} \mathrm{OH}\right)^{12}\right]$. IR (neat) 3412 (broad), 2929 (broad), 1709, 1634, 1440, 1305, 1162, 1077, $729 \mathrm{~cm}^{-1}$. HRMS $\left(\mathrm{MNa}^{+}\right)$, calcd: 983.37306, found: 983.37402. LC/MS (ESI) m/z: $\mathrm{R}_{\mathrm{t}}=4.93 \mathrm{~min},\left(\mathrm{MH}^{+}\right) 961$.

\begin{tabular}{|c|c|c|c|c|}
\hline \multirow{2}{*}{$\begin{array}{c}\text { H or } \\
\text { C }\end{array}$} & \multirow{2}{*}{\multicolumn{2}{|c|}{$\begin{array}{c}{ }^{1} \mathrm{H} \text { NMR } \\
(\mathrm{MeOD}, 500 \mathrm{MHz}) \delta\end{array}$}} & \multicolumn{2}{|c|}{$\begin{array}{c}{ }^{13} \mathrm{C} \text { NMR } \\
(\mathrm{MeOD}, 125 \mathrm{MHz}) \delta\end{array}$} \\
\hline & & & a part & b part \\
\hline 1 & $5.94(\mathrm{~s}, 1 \mathrm{H})$ & $5.97(\mathrm{~s}, 1 \mathrm{H})$ & 94.90 & 95.00 \\
\hline 3 & $7.52(\mathrm{~s}, 1 \mathrm{H})$ & $7.53(\mathrm{~s}, 1 \mathrm{H})$ & 155.18 & 155.18 \\
\hline 4 & - & - & 109.30 & 109.42 \\
\hline 5 & $4.00(\mathrm{dd}, J=8.9,4.4 \mathrm{~Hz}, 1 \mathrm{H})$ & $4.01(\mathrm{dd}, J=8.9,4.4 \mathrm{~Hz}, 1 \mathrm{H})$ & 31.90 & 32.03 \\
\hline 6 & $\begin{array}{l}2.67(\mathrm{dd}, J=13.9,4.6 \mathrm{~Hz}, 1 \mathrm{H}) \\
2.48(\mathrm{dd}, J=13.9,9.2 \mathrm{~Hz}, 1 \mathrm{H})\end{array}$ & $\begin{array}{l}2.74(\mathrm{dd}, J=14.1,4.5 \mathrm{~Hz}, 1 \mathrm{H}) \\
2.49(\mathrm{dd}, J=14.1,9.1 \mathrm{~Hz}, 1 \mathrm{H})\end{array}$ & 41.28 & 41.35 \\
\hline 7 & - & - & 173.09 & 173.18 \\
\hline 8 & $6.10(\mathrm{q} b r, J=7.3 \mathrm{~Hz}, 1 \mathrm{H})$ & $6.13(\mathrm{q} b r, J=7.3 \mathrm{~Hz}, 1 \mathrm{H})$ & 124.72 & 124.85 \\
\hline 9 & - & - & 130.63 & 130.72 \\
\hline 10 & $1.75(\mathrm{dd}, J=7.1,1.3 \mathrm{~Hz}, 3 \mathrm{H})$ & $1.76(\mathrm{dd}, J=7.1,1.3 \mathrm{~Hz}, 3 \mathrm{H})$ & 13.80 & 13.84 \\
\hline 11 & - & - & 168.58 & 168.61 \\
\hline OMe & $3.71(\mathrm{~s}, 3 \mathrm{H})$ & $3.71(\mathrm{~s}, 3 \mathrm{H})$ & 51.95 & 51.96 \\
\hline 1' & $4.80(\mathrm{~d}, J=7.7 \mathrm{~Hz}, 1 \mathrm{H})$ & $4.80(\mathrm{~d}, J=7.7 \mathrm{~Hz}, 1 \mathrm{H})$ & 100.62 & 100.70 \\
\hline 2' & $3.26-3.36(\mathrm{~m}, 1 \mathrm{H})$ & $3.26-3.36(\mathrm{~m}, 1 \mathrm{H})$ & 74.71 & 74.77 \\
\hline 3' & $3.40(\mathrm{t}, J=8.9 \mathrm{~Hz}, 1 \mathrm{H})$ & $3.40(\mathrm{t}, J=8.9 \mathrm{~Hz}, 1 \mathrm{H})$ & 77.92 & 77.92 \\
\hline 4' & $3.26-3.36(\mathrm{~m}, 1 \mathrm{H})$ & $3.26-3.36(\mathrm{~m}, 1 \mathrm{H})$ & 71.50 & 71.60 \\
\hline 5 ' & $3.26-3.36(\mathrm{~m}, 1 \mathrm{H})$ & $3.26-3.36(\mathrm{~m}, 1 \mathrm{H})$ & 78.48 & 78.54 \\
\hline $6^{\prime}$ & $\begin{array}{l}3.88(\mathrm{dd}, J=11.9,2.0 \mathrm{~Hz}, 1 \mathrm{H}) \\
3.65(\mathrm{dd}, J=11.9,5.5 \mathrm{~Hz}, 1 \mathrm{H})\end{array}$ & $\begin{array}{l}3.89(\mathrm{dd}, J=11.9,2.0 \mathrm{~Hz}, 1 \mathrm{H}) \\
3.66(\mathrm{dd}, J=11.9,5.5 \mathrm{~Hz}, 1 \mathrm{H})\end{array}$ & 62.79 & 62.94 \\
\hline 1” & $2.02(1$ & , 1H) & & \\
\hline 2” & $1.63(\mathrm{I}$ & , $1 \mathrm{H})$ & & \\
\hline
\end{tabular}

\footnotetext{
${ }^{12}$ Tanahashi, T.; Takenaka, Y.; Nagakura, N.; Nishi, T. chem.. Pharm. Bull. 2000, 48, 81200.
} 


\begin{tabular}{|c|c|c|}
\hline 3” & $1.77-1.89(\mathrm{~m}, 1 \mathrm{H})$ & 52.69 \\
\hline $\mathbf{4 ”}$ & $4.98(\mathrm{~m}, 1 \mathrm{H})$ & 81.09 \\
\hline $\mathbf{5 ”}$ & $\begin{array}{c}1.85(\mathrm{dd} b r, J=13.7,6.5 \mathrm{~Hz}, 1 \mathrm{H}) \\
1.49(\mathrm{ddd}, J=13.7,11.5,5.4 \mathrm{~Hz}, 1 \mathrm{H})\end{array}$ & 41.66 \\
\hline $\mathbf{6 ”}$ & $1.08(\mathrm{~d}, J=6.5 \mathrm{~Hz}, 3 \mathrm{H})$ & 19.95 \\
\hline $\mathbf{7 ”}$ & $4.15(\mathrm{dd}, J=11.0,5.6 \mathrm{~Hz}, 1 \mathrm{H})$ & 69.22 \\
\hline $\mathbf{8 ”}$ & $3.97(\mathrm{dd}, J=11.0,6.9 \mathrm{~Hz}, 1 \mathrm{H})$ & 39.15 \\
\hline $\mathbf{9 ”}$ & $1.77-1.89(\mathrm{~m}, 1 \mathrm{H})$ & 15.00 \\
\hline $\mathbf{1 0}$ & $0.95(\mathrm{~d}, J=6.5 \mathrm{~Hz}, 3 \mathrm{H})$ & 66.52 \\
\hline
\end{tabular}

Significant COSY correlations: $\mathrm{H}-1$ ” $\Leftrightarrow \mathrm{H}_{2}-5$ ” ; H-1" $\Leftrightarrow \mathrm{H}_{3}-6$ ” ; H-2” $\Leftrightarrow H-3$ ” ; H-2” $\Leftrightarrow H-$

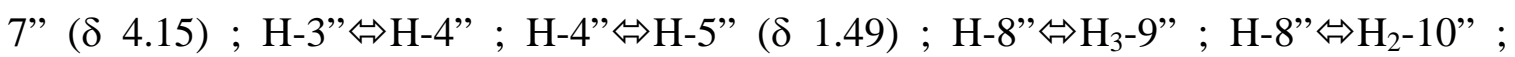
Significant HMBC correlations: H-4” $\Rightarrow$ C-7 ; H-4” $\Rightarrow C-8$ ” ; $\mathrm{H}_{3}-6$ ” $\Rightarrow$ C-1” ; $\mathrm{H}_{3}-6$ ” $\Rightarrow \mathrm{C}-2$ ” ;

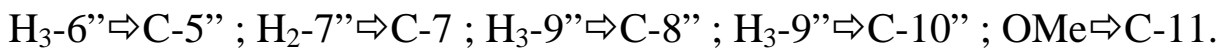

These spectra correspond with the ones described in the literature. ${ }^{11}$

\section{3-Selected ${ }^{1} \mathrm{H}$ and ${ }^{13} \mathrm{C}$ spectra for compounds 1 to 15 and ${ }^{31} \mathrm{P}$ for compound 5:}


66580.57

OELET'

$66 \mathrm{rgt} \cdot \mathrm{T}$

rocte

ट८095. $5-$

998LC'

9टย6อ ' 5

$088 \mathrm{e} \cdot \mathrm{r}$

L956.'

SOOEO 2 -

9E $\angle E O O^{\circ}-7$

$0 \mathrm{~T} \angle 0^{\circ} 2-1$

00105'

vitric 27

$\checkmark 92 \angle 1 \cdot 2-2$

6860E' 27

ОЕВटE' 2

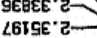

OE $\angle 9 E$ ' $2-$

689LE' 2

- L9S6E' 2

- TLEzV Z

Geer $2-$

s009t ' $2-1$

- L9r8s' 2 -

* 80r rs' 2 -

น9r09. $2-$

ESSEL 2 -

Ort 92 ' $2-$

ปEน 19

เร659.

89PEg' $\mathrm{E}$

06259 ' 2

$\checkmark 689 L^{\prime} \varepsilon$

$606 \angle 1 \cdot 8$

EE26L $5-$

gterr.b-

вETET $\cdot$

E9זट⿱艹

8SIS"

LZELV' '

59L6V $\square$

cotsit.

G)Sit 's

$\angle D O L \sigma^{\circ} \mathrm{S}$

0E909' 9

L0829' 9

$6 \angle \angle 49.9$

$6 \% \angle 59.9$

19699' 9

168 $\angle 9.9$

$\varepsilon \angle 869$ '

टESGC. $\angle 2$

TStLC.

Erc口2.

Ersoe -

$\angle \angle$ LCE ' $\angle-$

6LTEE' $2-$

9टtEE' 2

OLBEE' 2

ELIVE' $L$

GEO9E.

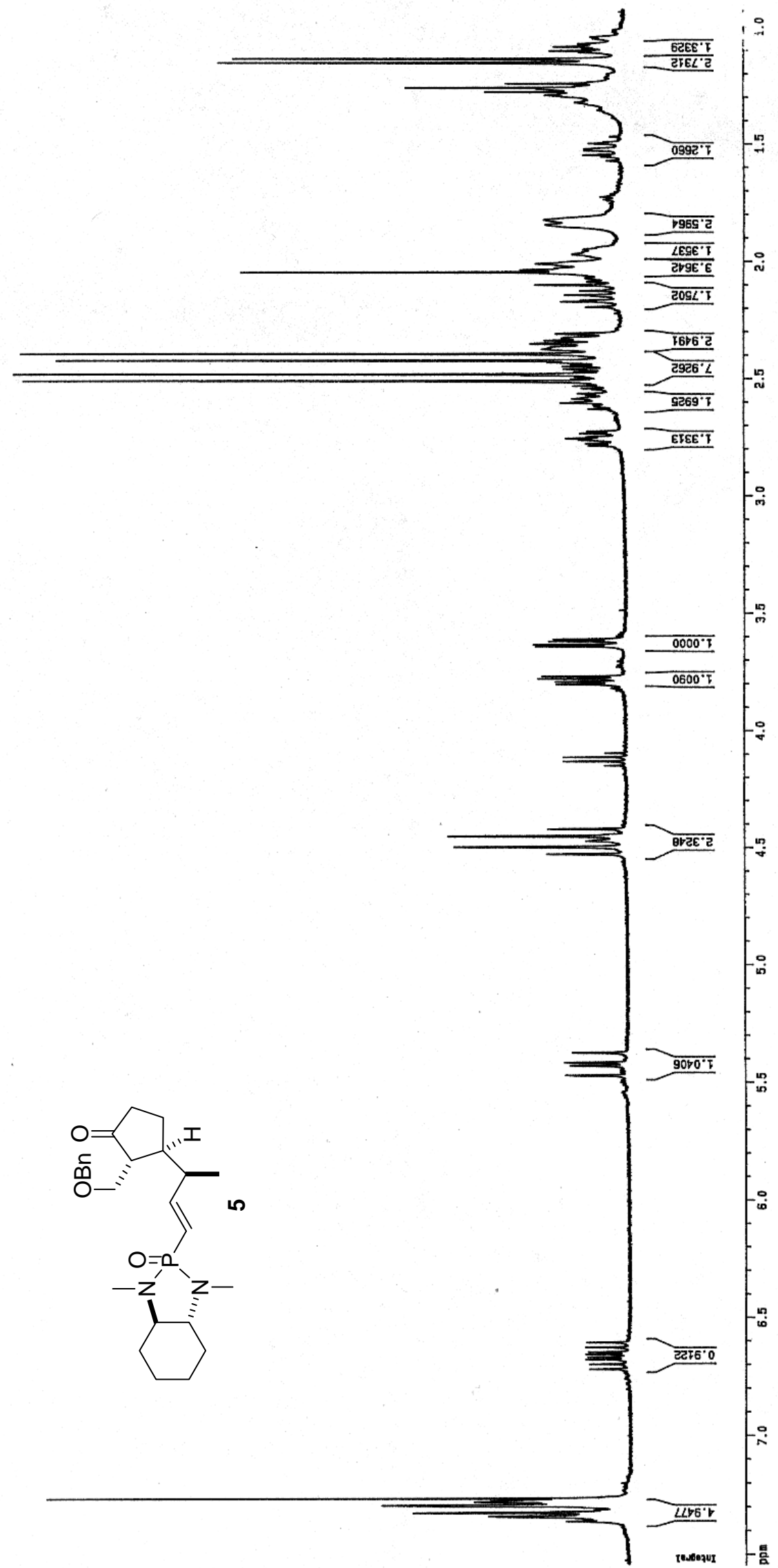


$90^{\circ} \mathrm{BI}$

टा8 22

$086^{\circ} \mathrm{EC} \longrightarrow$

060.82

$960^{\circ} \angle 2$

$02 V^{\prime} 82=$

$\mathrm{StG} \cdot 8 \mathrm{c}$

Org.82

$650.8 \mathrm{E}$

$06 \mathrm{v}^{\circ} \mathrm{Ob} \longrightarrow$

$\angle E L^{\circ} O b=$

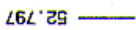

897. $69>$

$\angle E G^{\circ} E 9$

9rG' $t 9$

$698^{\circ} \angle 9$

вเ

$809 \cdot 9 L$

EOO $\angle L>$

8St $\angle L$

ITE $\angle 21-$

SGE $\angle 2 T \longrightarrow$

$\angle E T^{\circ}$ Bट -

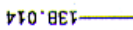

$\angle 9 L^{\circ} \nabla \mathrm{GI}$

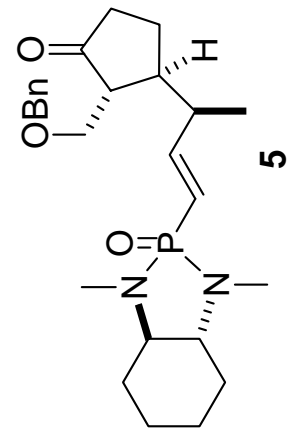

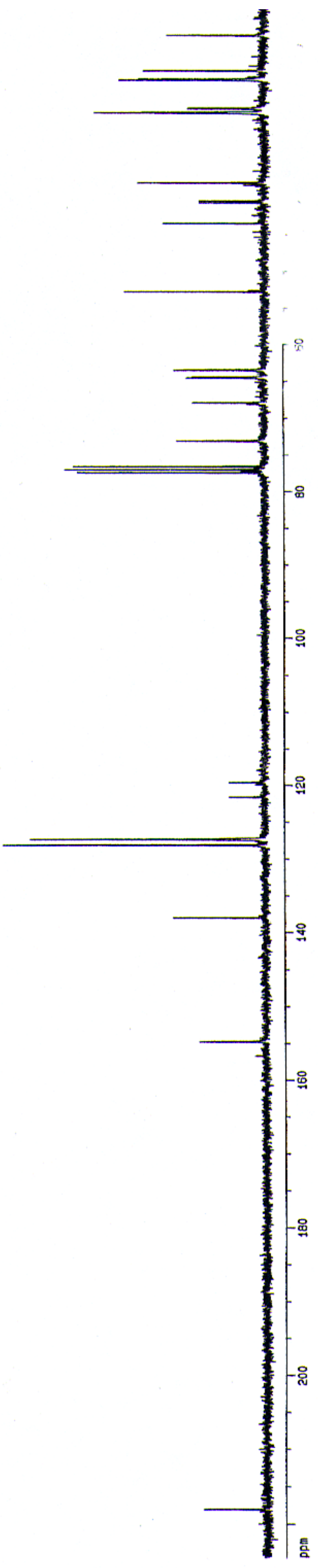

urdd 

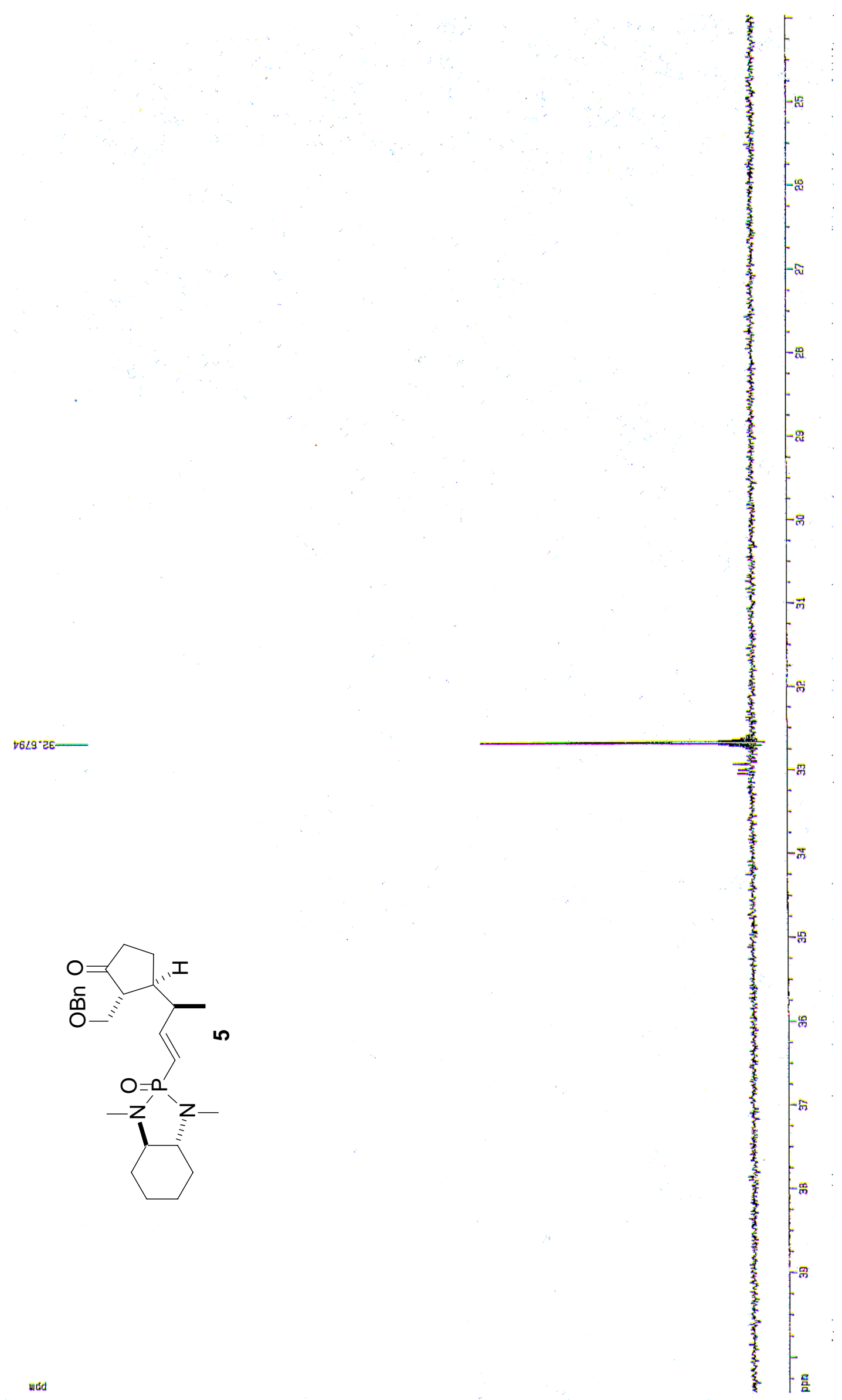


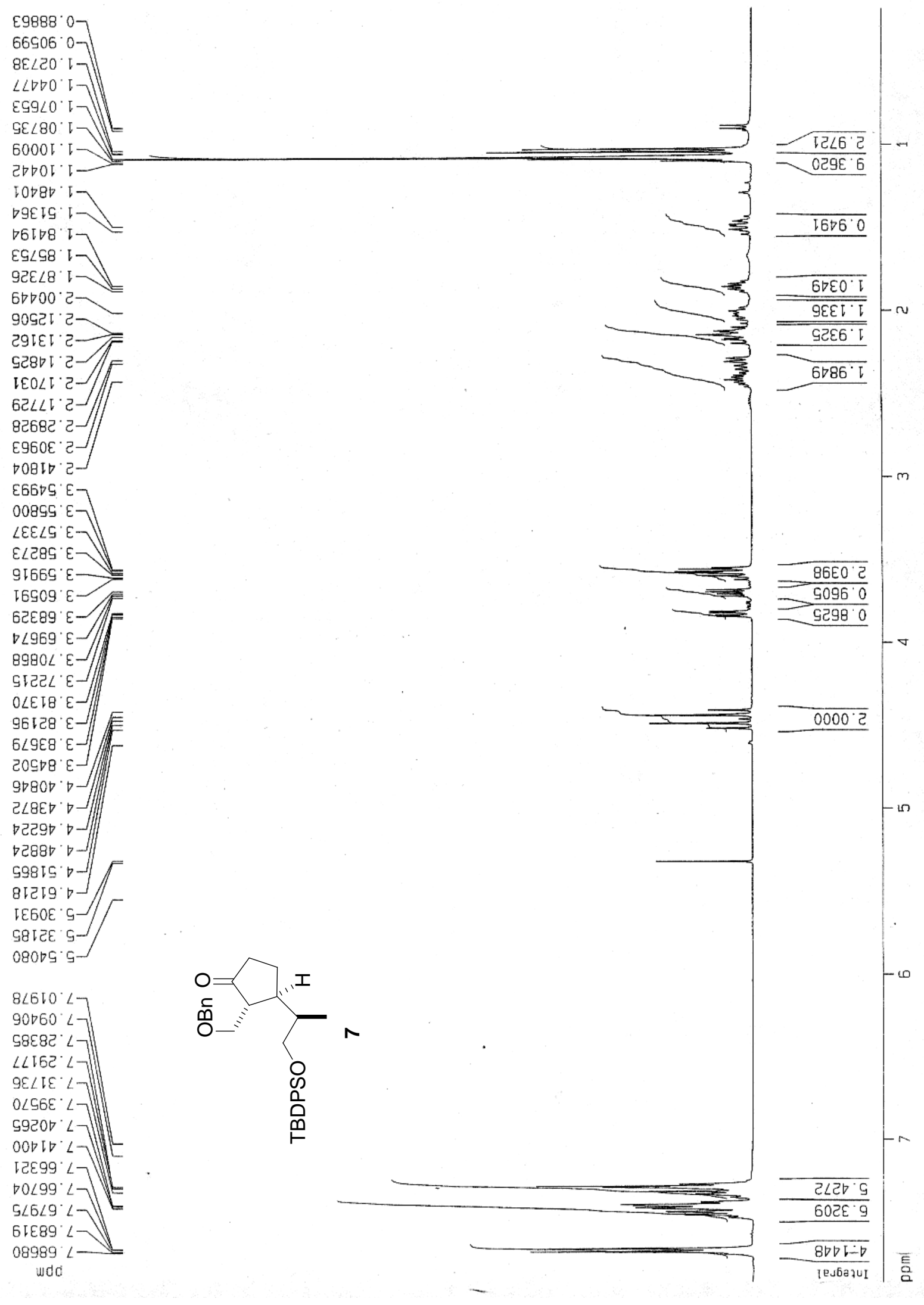




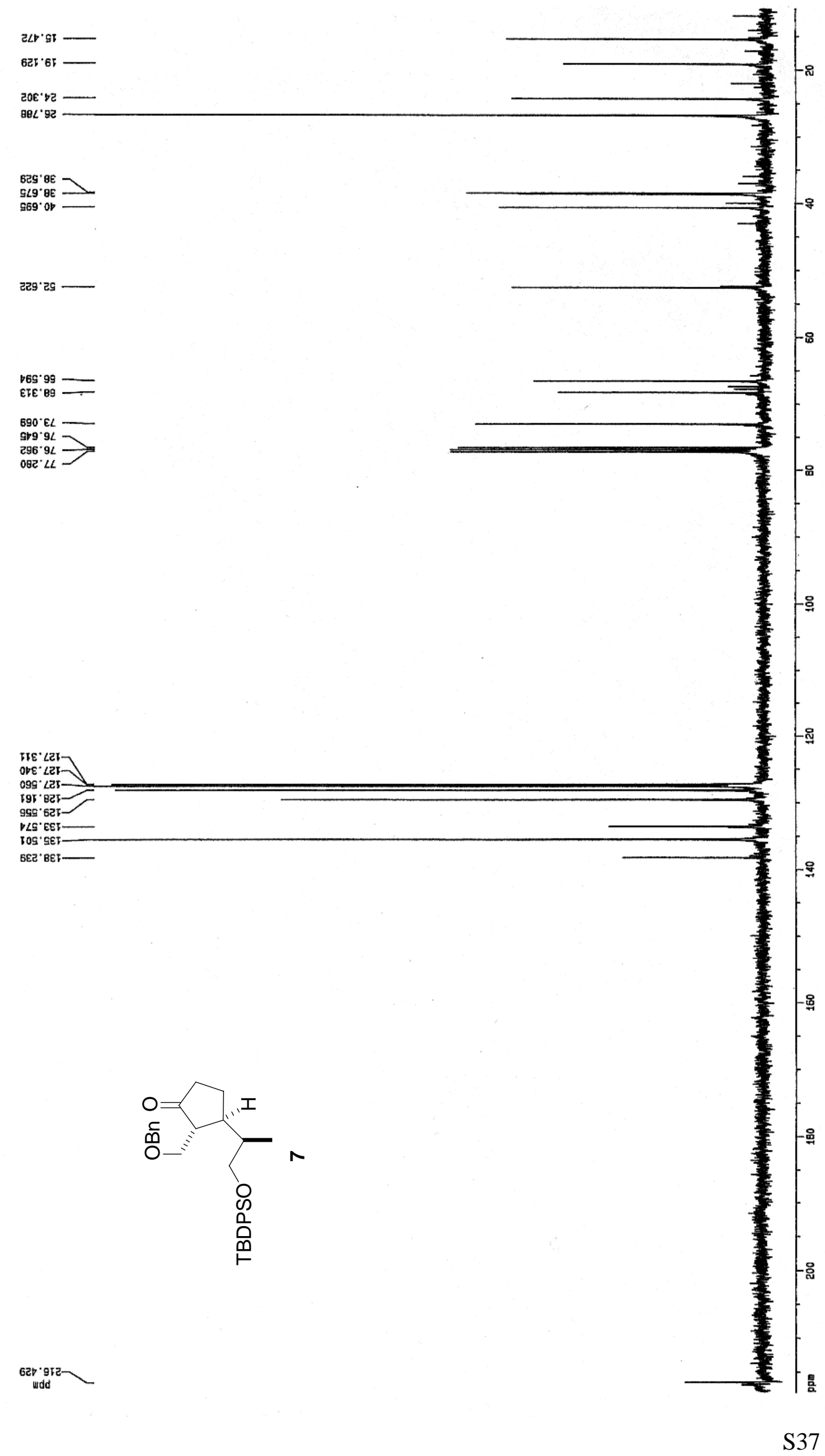




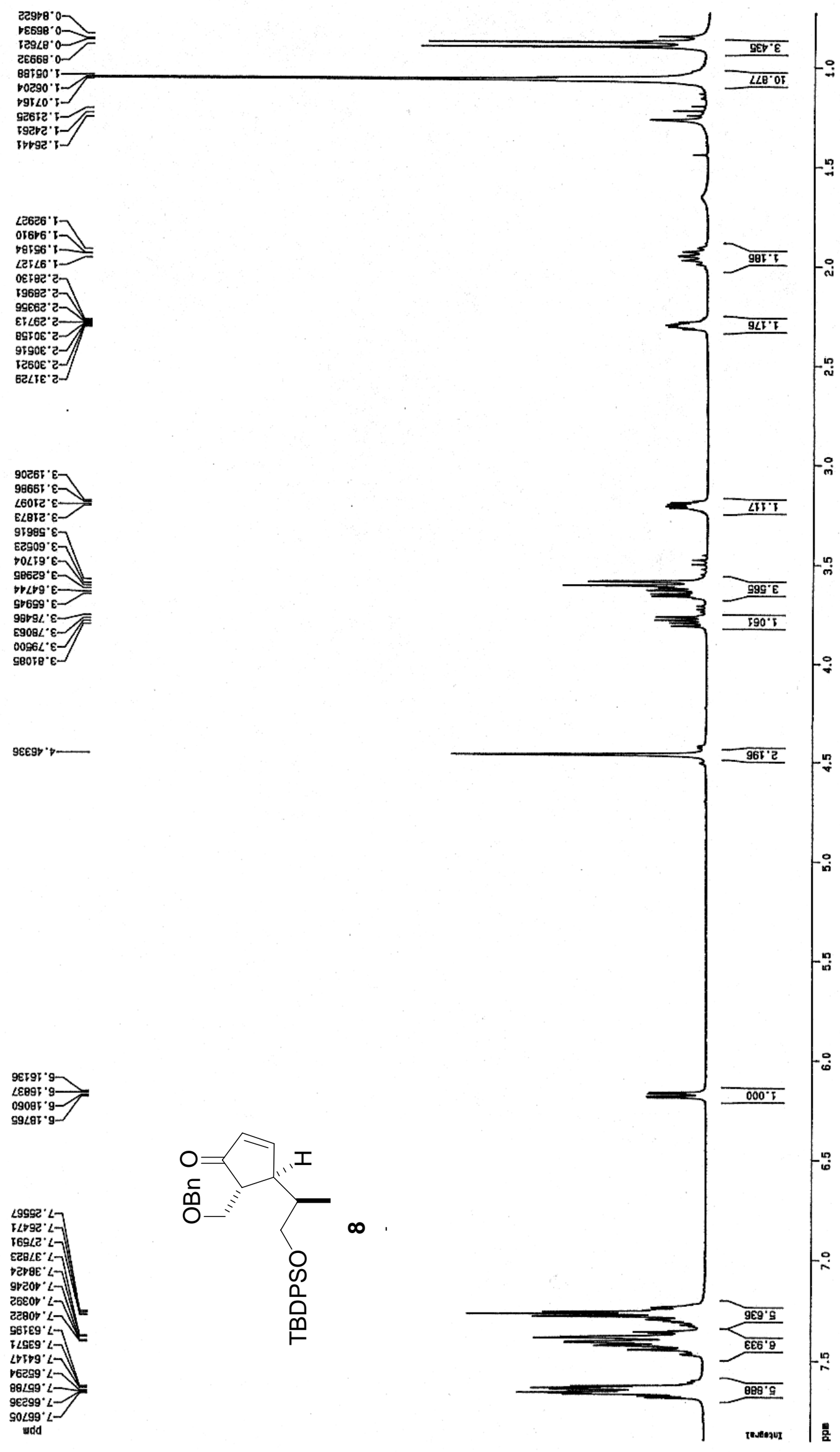




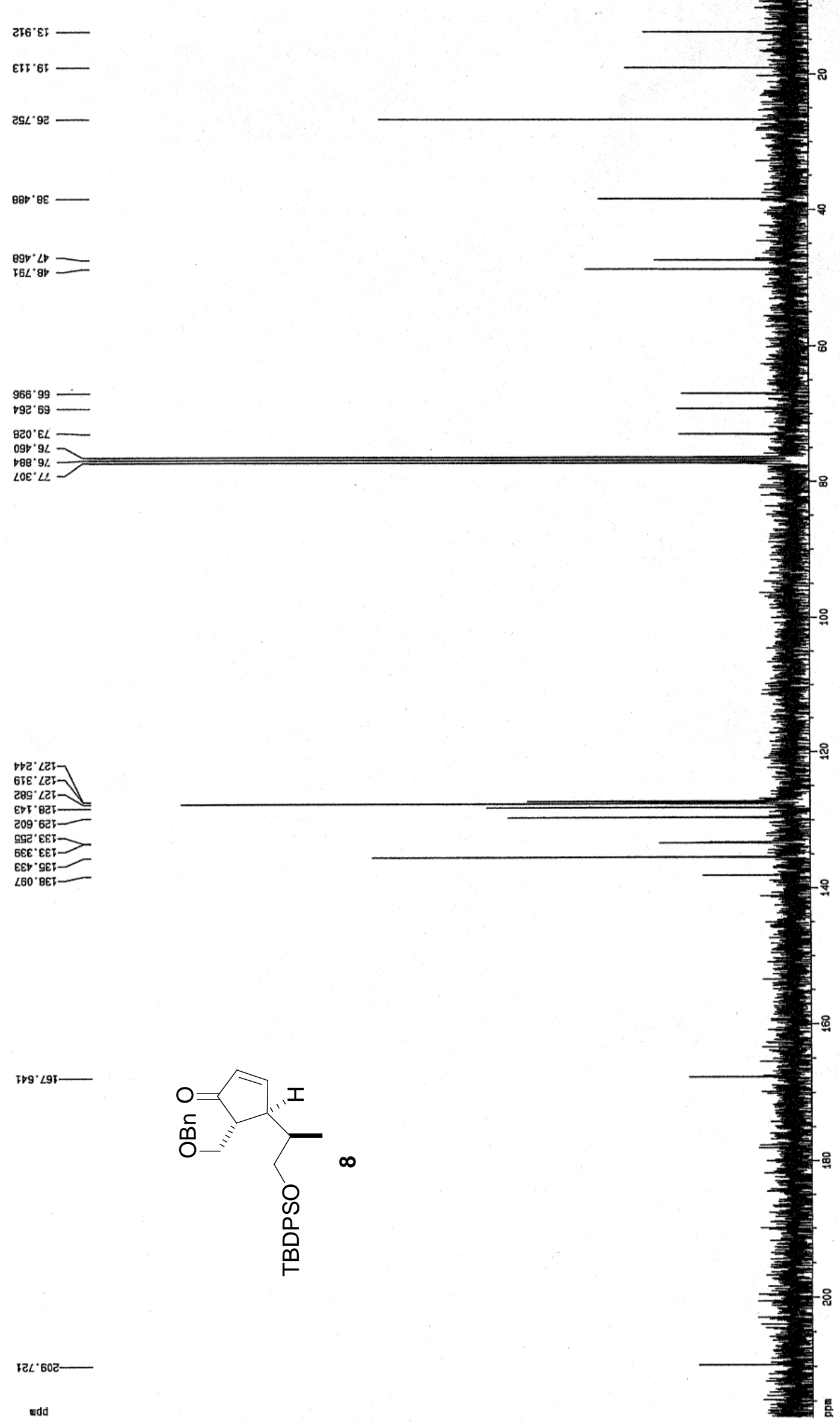




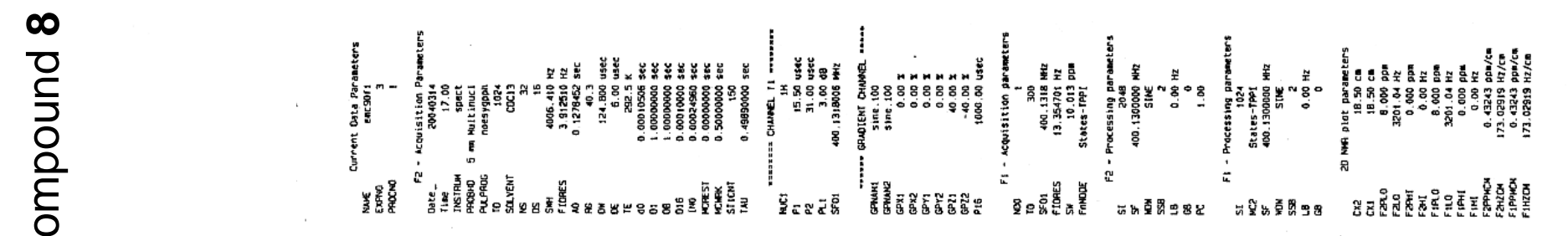

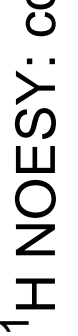
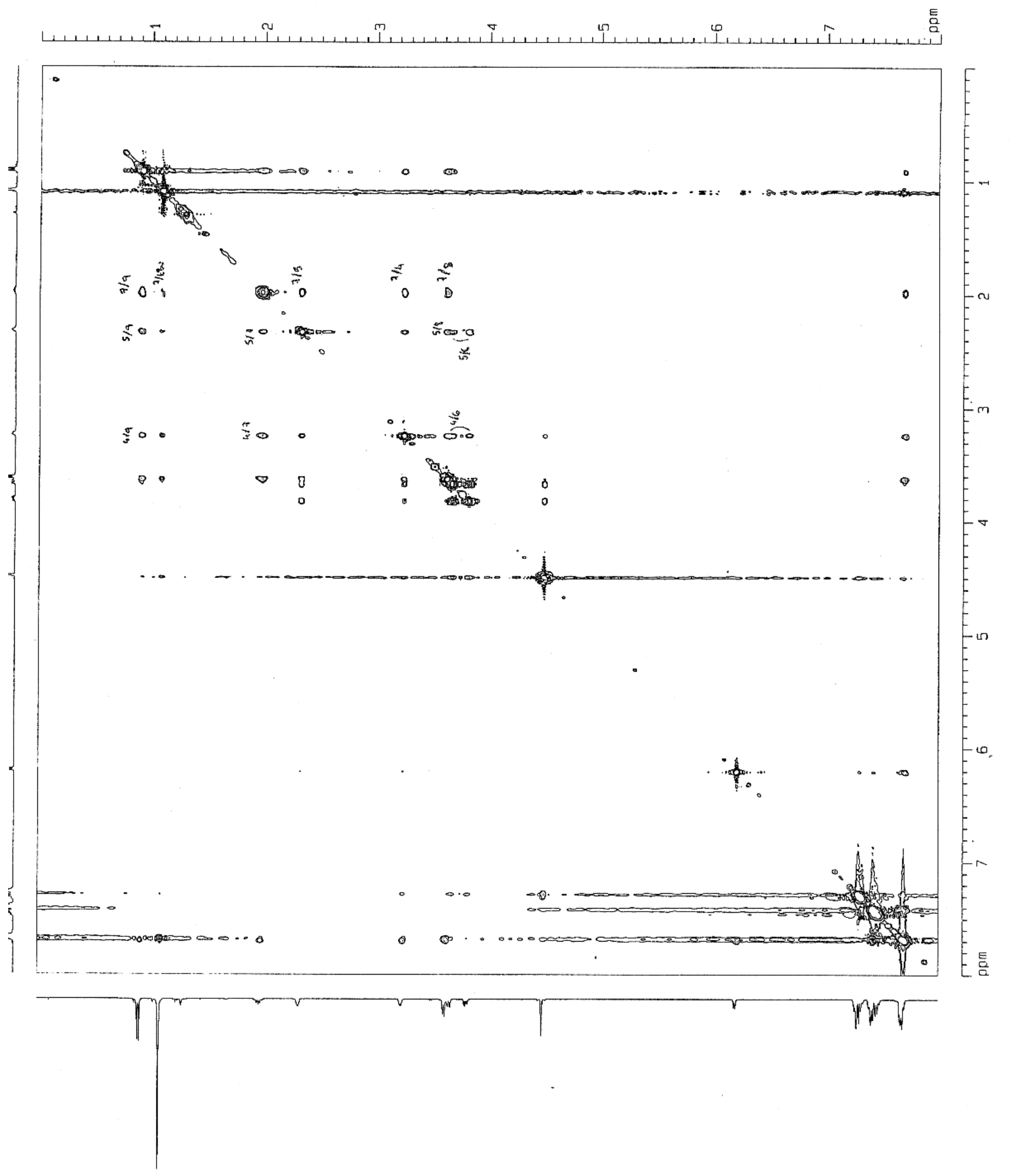


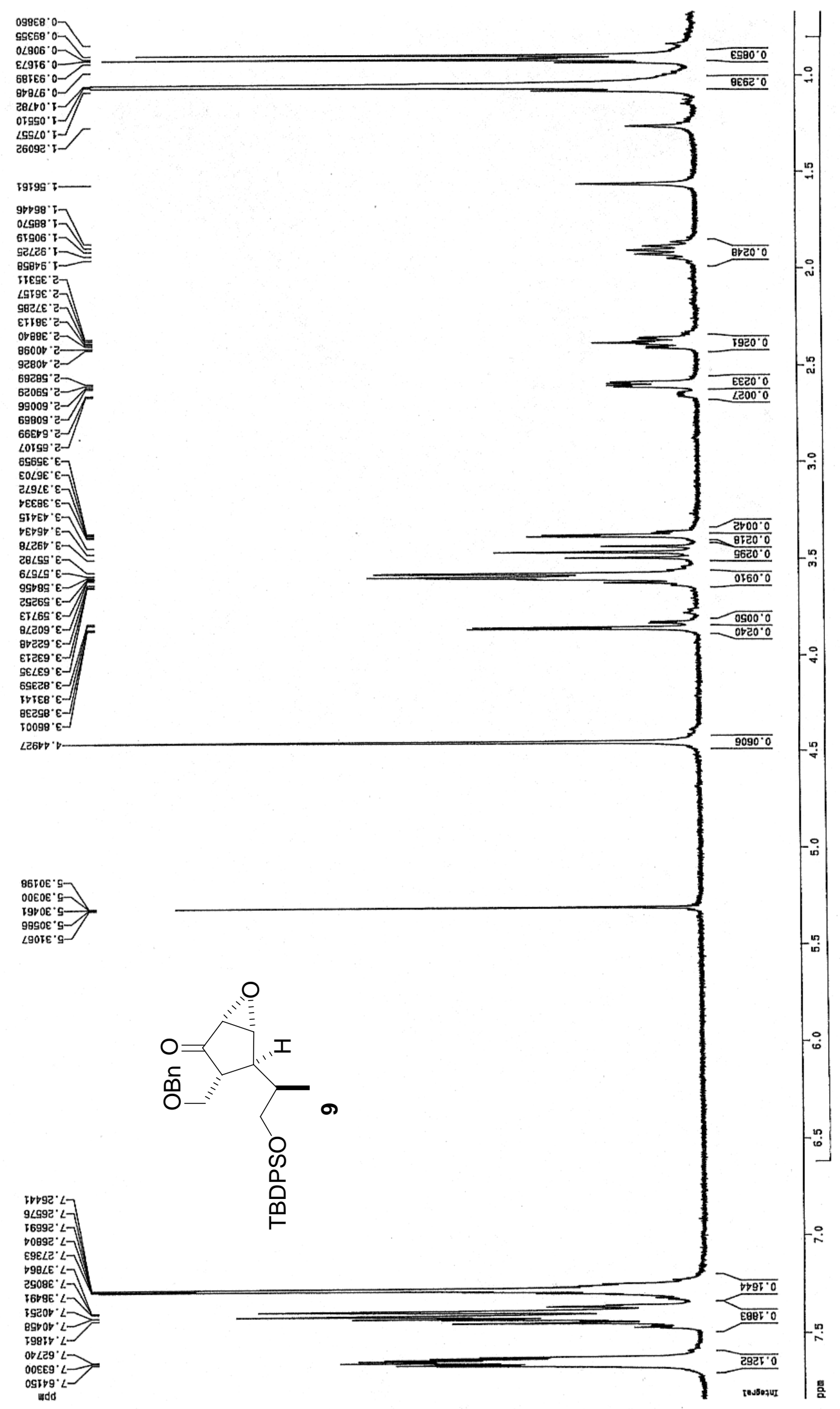



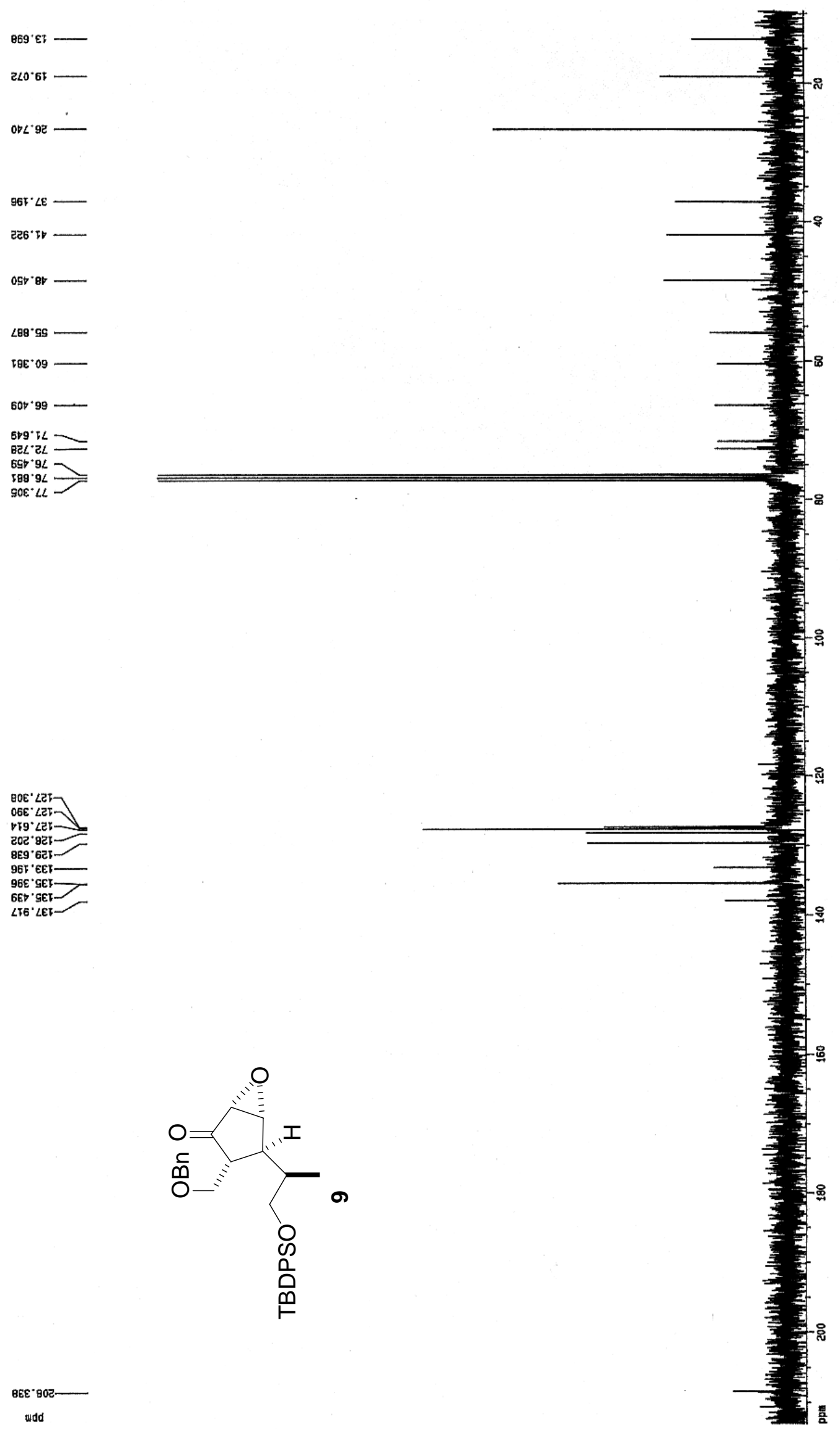


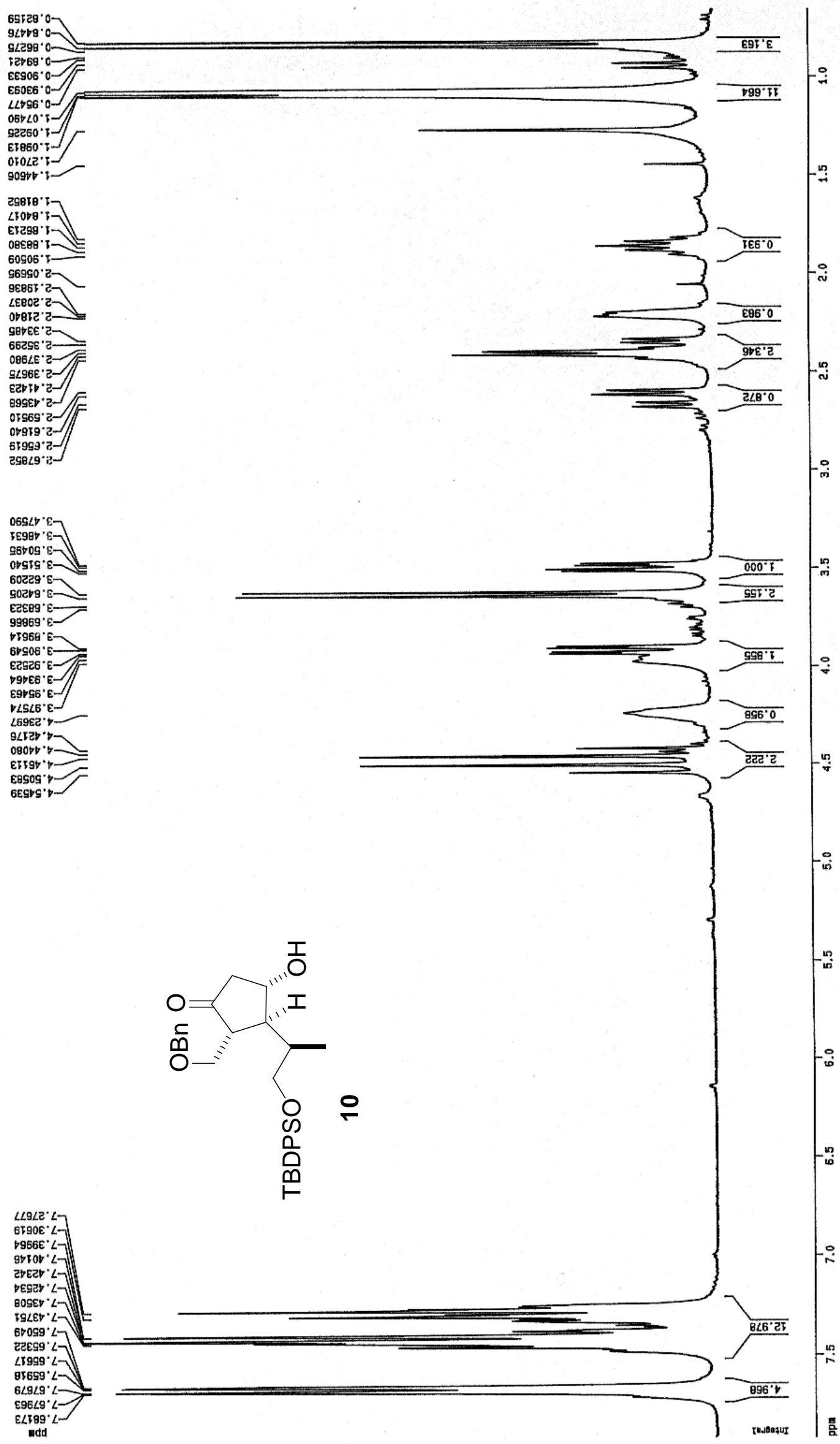


$96 L^{\circ} \nabla 5$

950.65

$62 L \cdot 92$

L29' $9 \varepsilon$

EIE' 67

IES'US

\section{(1)}

$880^{\circ} \angle 9-$
$0 \angle 9 \cdot 69-$

$0 \angle 9^{\circ} 69-$

टटट ' $\angle L$

$987^{\circ} \mathrm{EL}-$

606 ' $9 L$

EEE' $L L$

OBS. $\angle C F$

$699 . \angle 2 F-$

g大: '

OTE 'Bet-

(5).

OS6 ' टEV

GET' $\angle E T-$

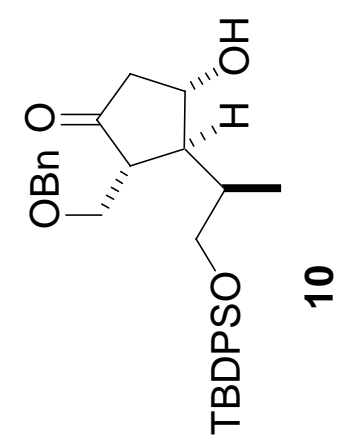

$\theta L V^{\circ} \angle I Z$

wdd 


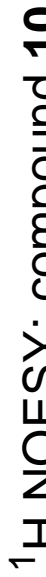

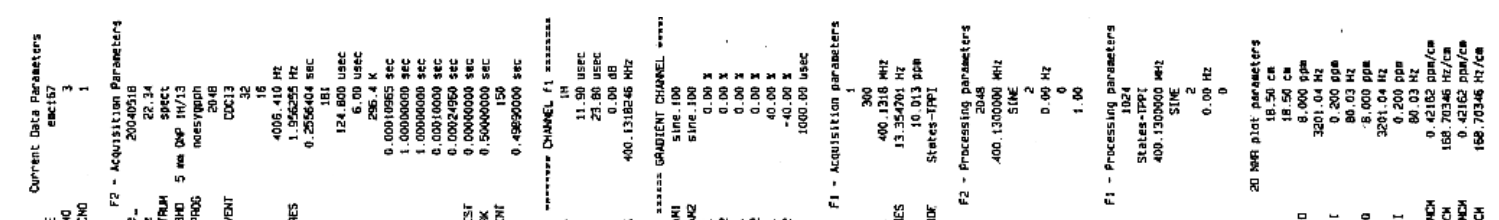

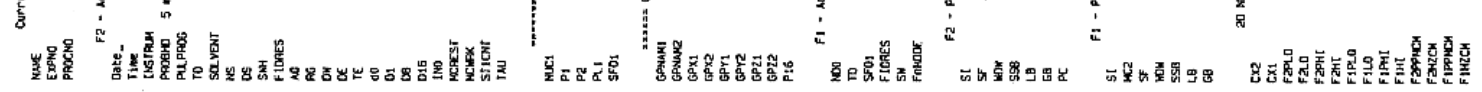
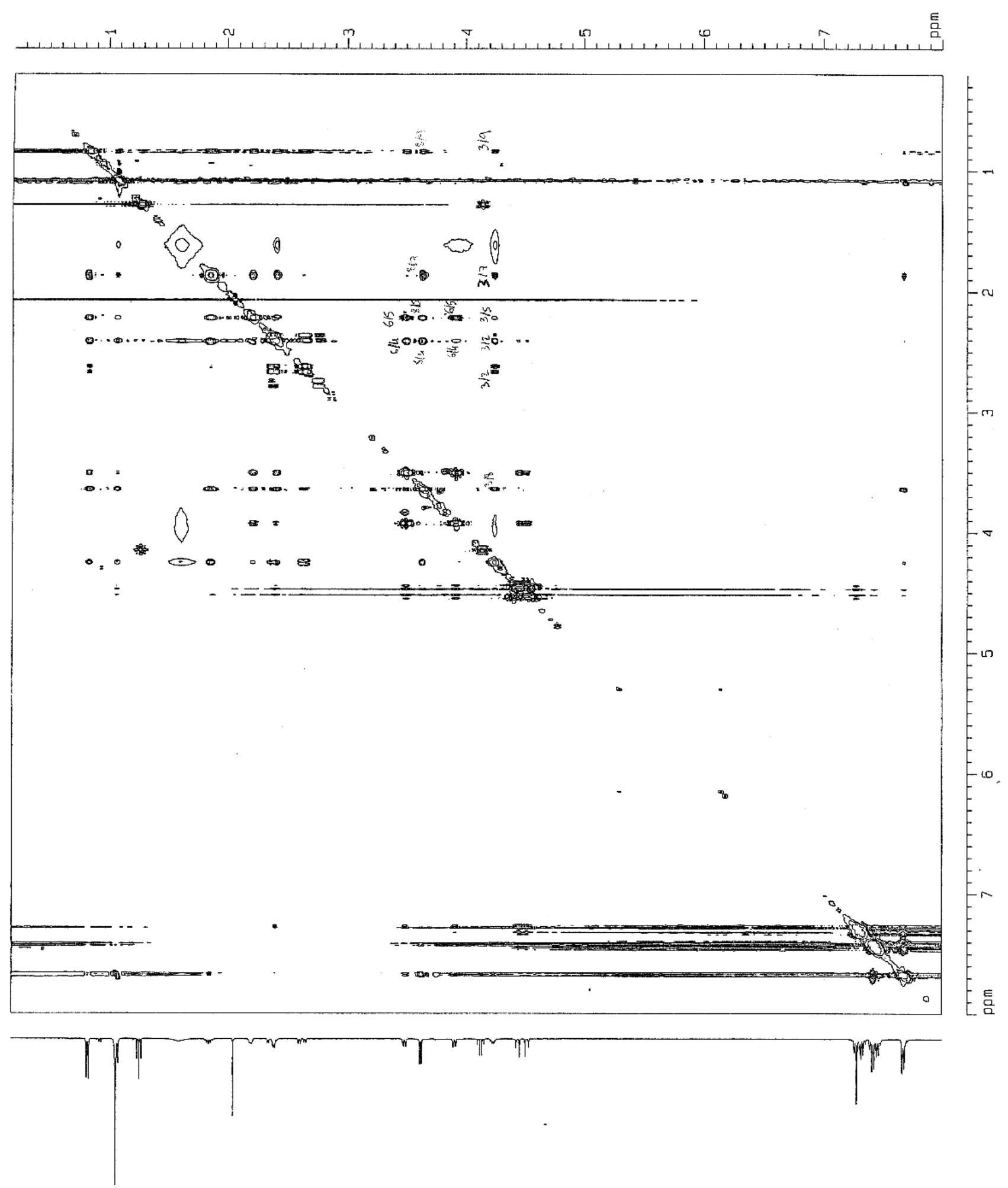


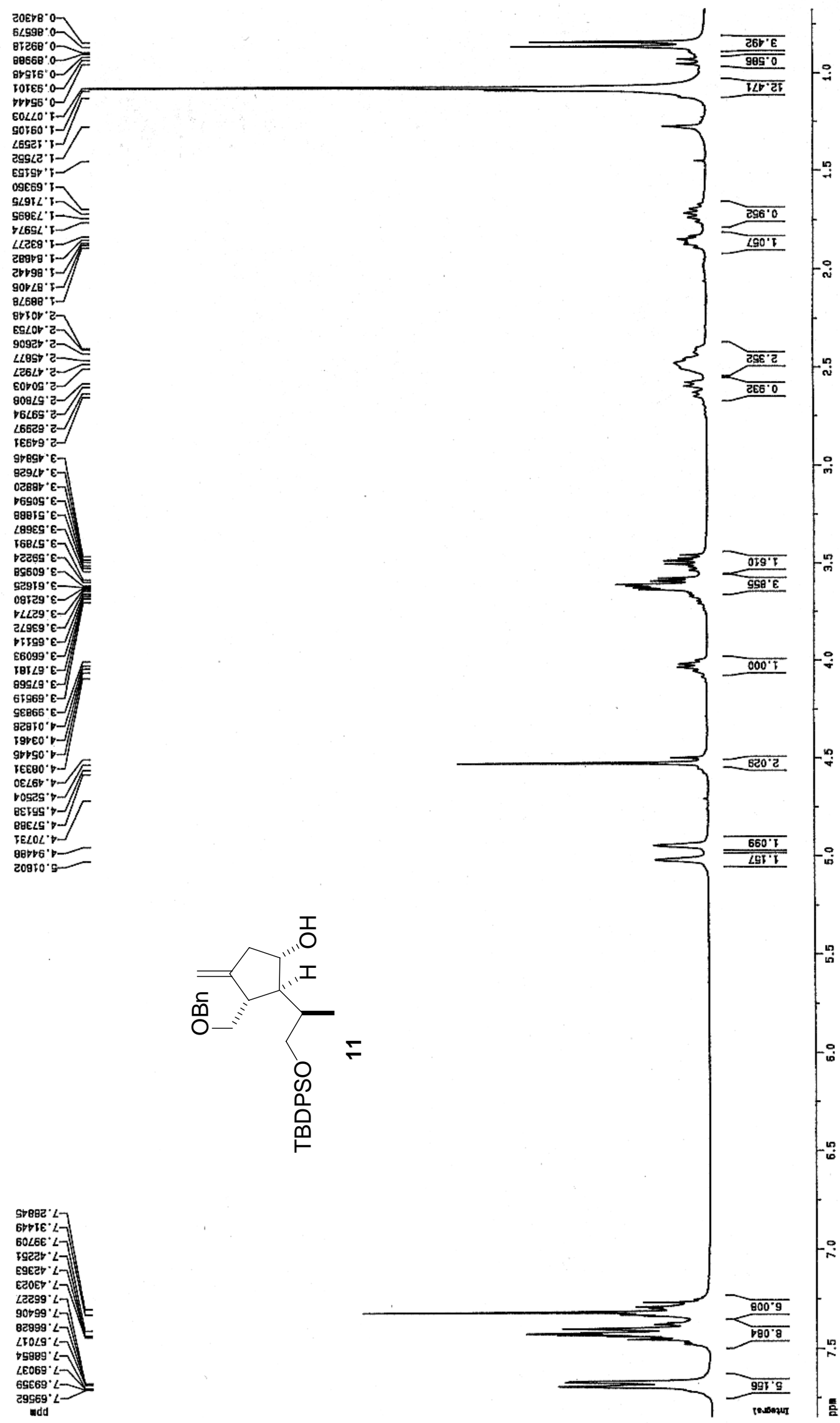



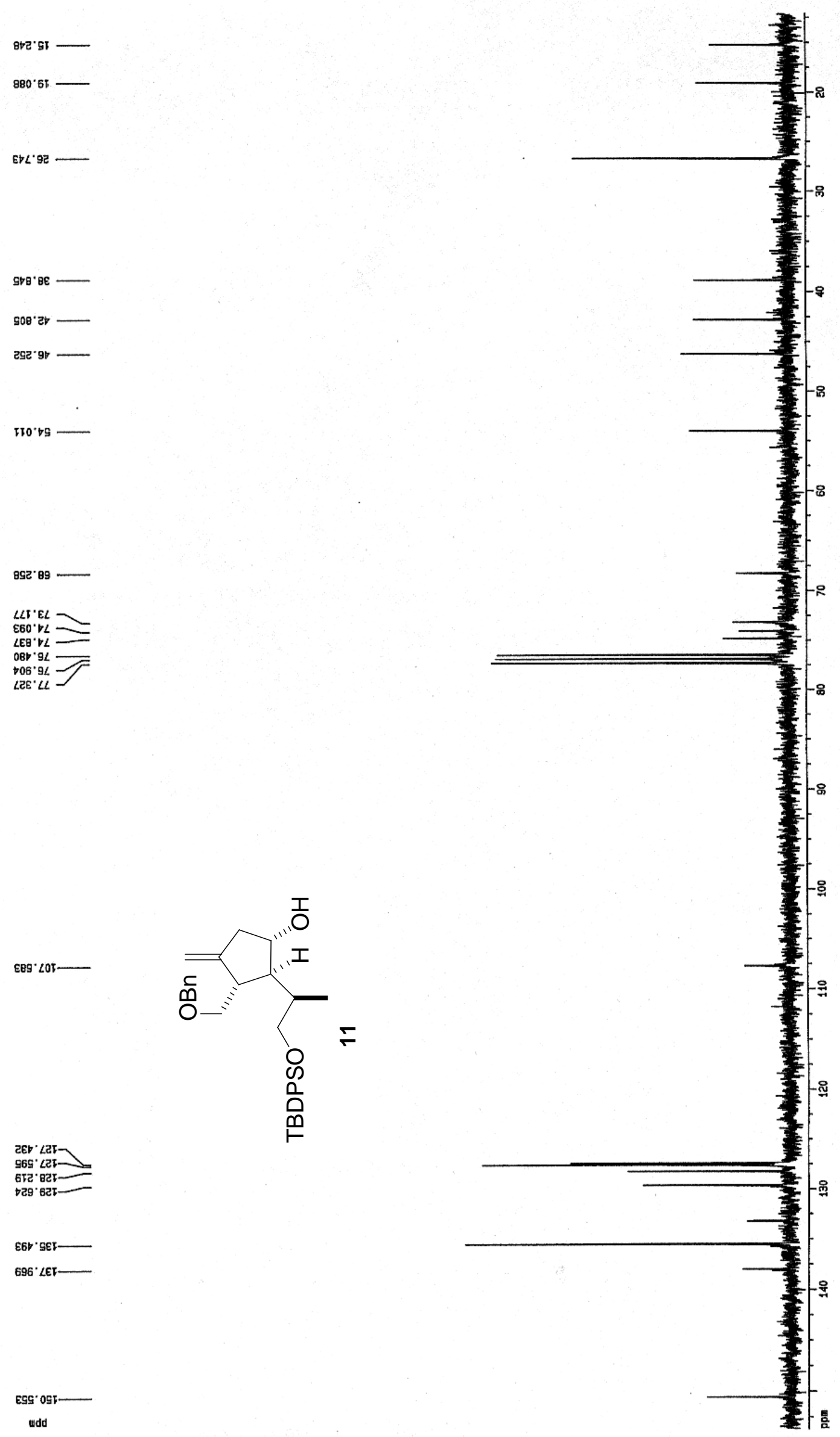

ESG. 055 -

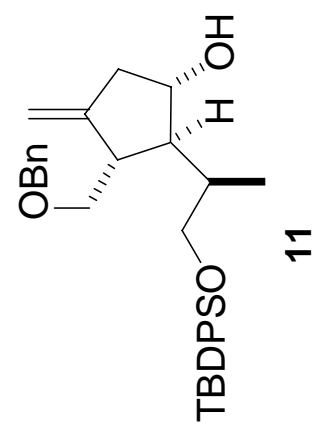

$2 E V^{\circ} \angle 25-1$
S65. $\angle 25-$
65.

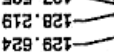

$\varepsilon 6 b \cdot s e r-$

$696^{\circ} \mathrm{LER}$ 


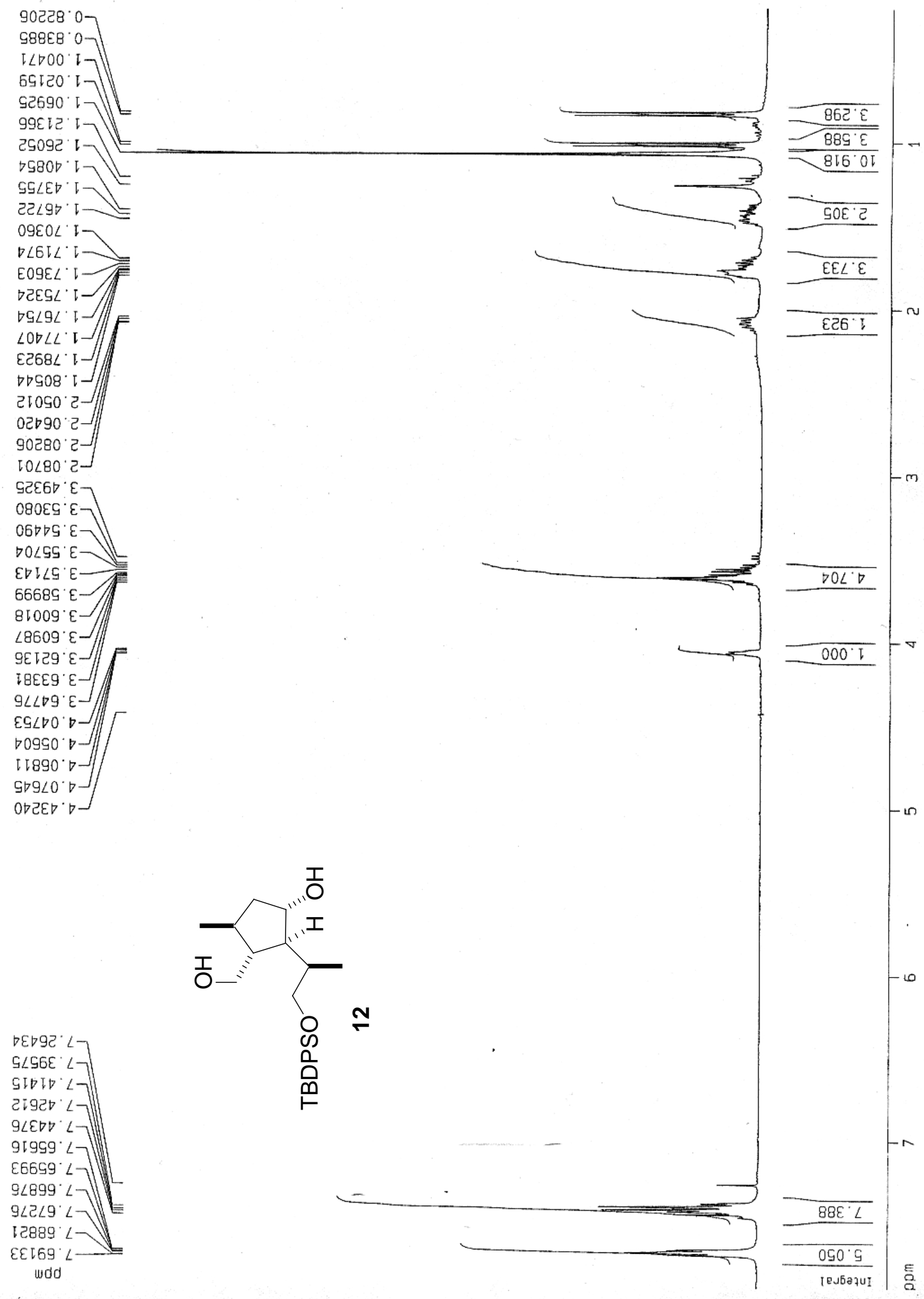




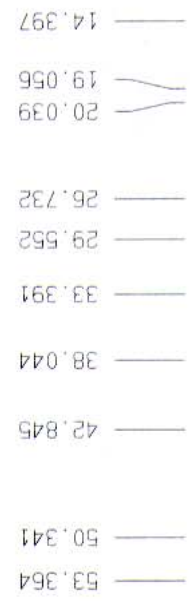

$\nabla 9 E^{\prime} E G-$

$66 E^{\circ} 99$

$199^{\circ} \angle 9$

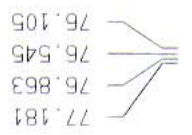

$0 \angle 9^{\circ} \angle 2 T=$

E09 $527 \longrightarrow$

$9 E 9.625$

$O \nabla \mathcal{C}^{\prime} E E$

टLV SET

wdd

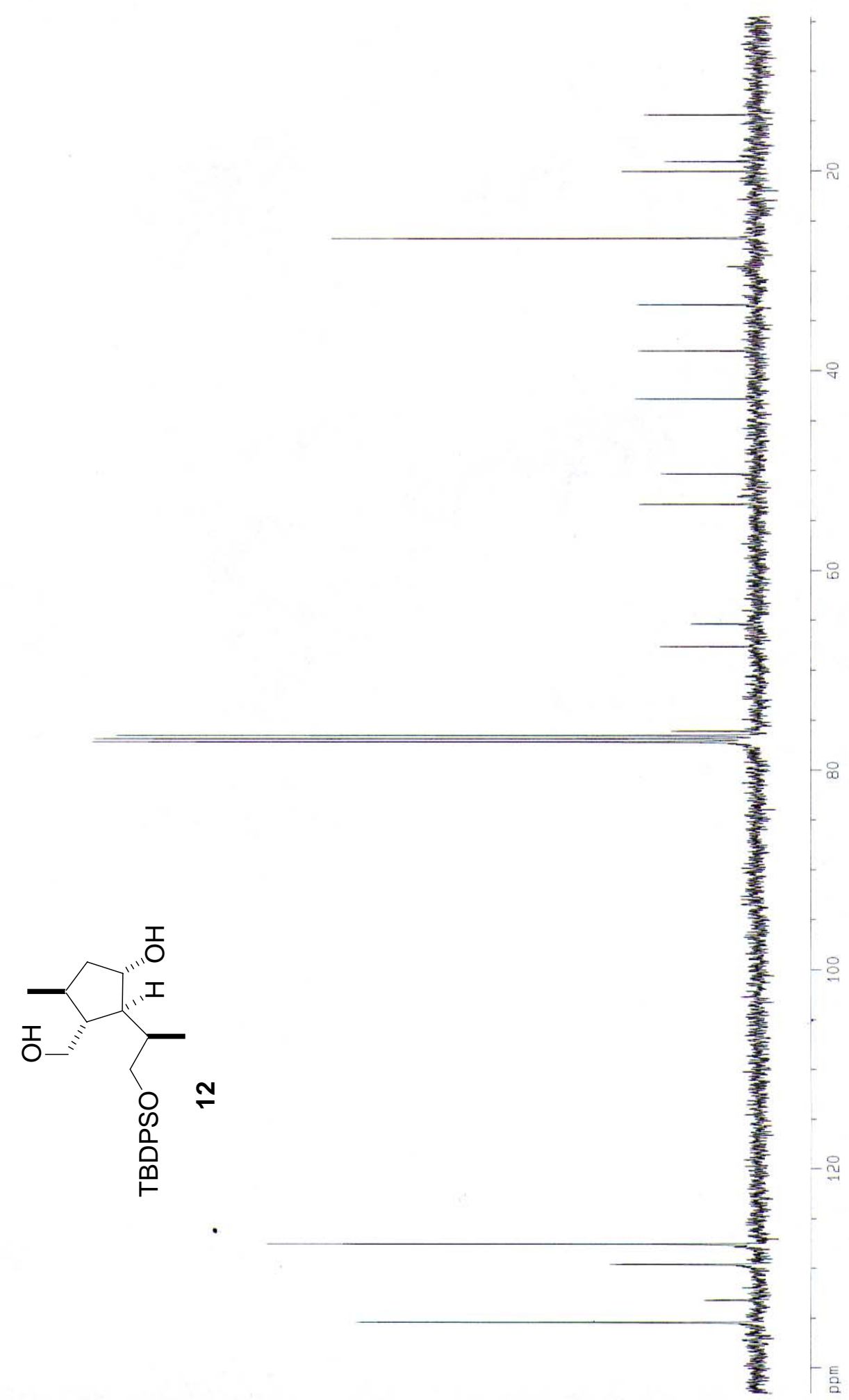




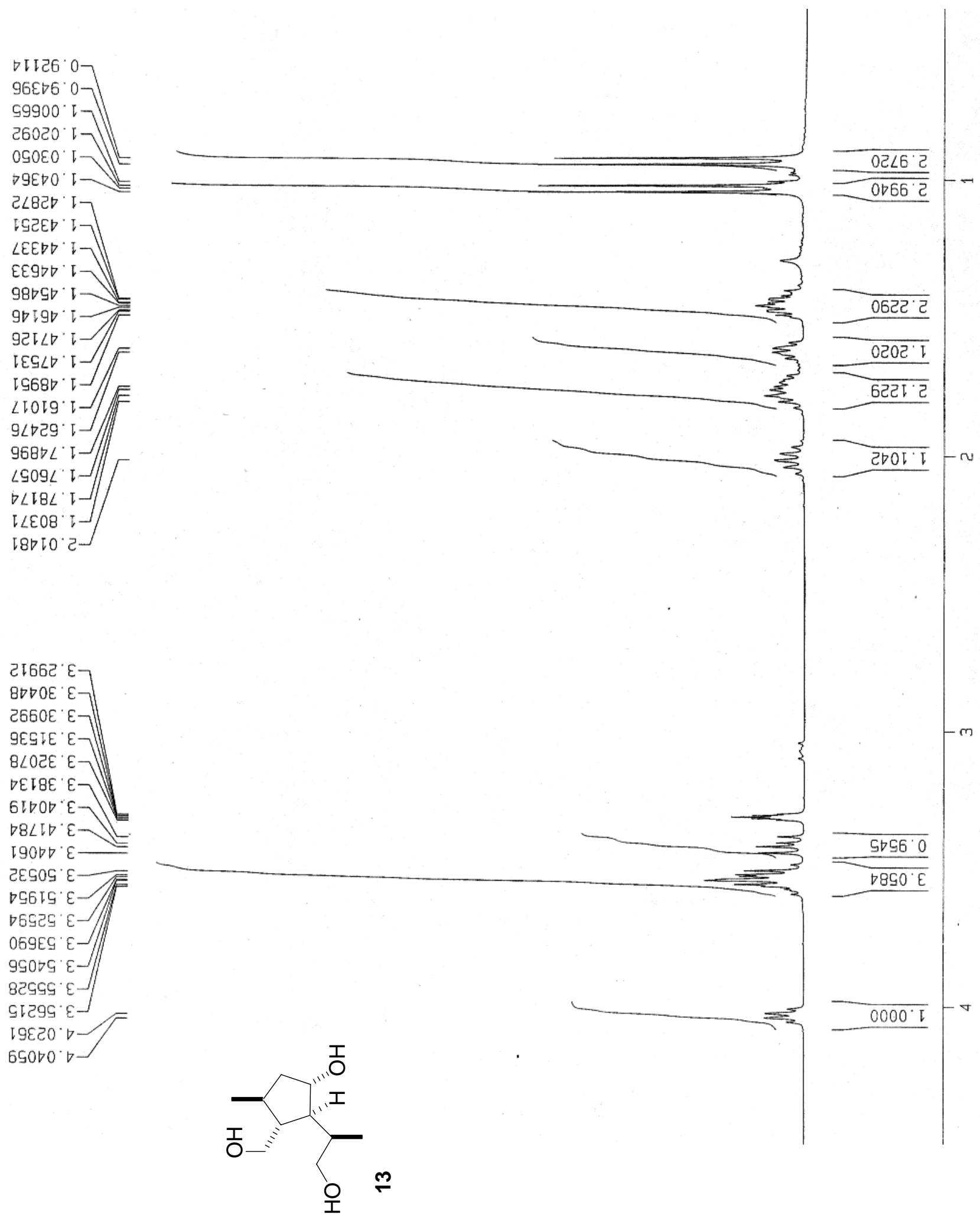


$\triangleright G G D^{\circ} \varepsilon$

99G6. $\mathrm{EI}$

629०००I-

$9999^{\circ} 02-$

EE88.0Z

$2 \nabla 80^{\circ} \angle 2$

$\nabla 69 L \cdot O E$

$29 \angle 0^{\circ} \mathrm{gE}=$

ट8टL' $\triangle E-$

$\left\lceil\angle \angle 0^{\circ} B \varepsilon\right.$

IE $\angle 2 \cdot 6 E-$

ट $\angle 69^{\circ} E V 7$

$\varepsilon \nabla \nabla 6^{\circ} \varepsilon \nabla-$

$\checkmark 6 \nabla[\cdot 8 \nabla]$

$9 \varepsilon \varepsilon \nabla \cdot 8 \nabla>$

$\varepsilon \angle L \angle \circ 8 \nabla$

110060

$6 \nabla 8 \mathrm{C}^{\prime} 6 \mathrm{~b}$

$8899^{\prime} 60$

จ298'60

$689 e^{\prime}$ ' Is

टटIB. 29

OLBO० $0 \mathrm{C}$

IIGट $\nabla 9$

टाटन०

$\angle 982 \cdot 59$ $\mathrm{G} \angle B \angle{ }^{\circ} 99-$

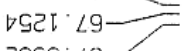

ट8E9 $\angle 9$

BED8 $E L$

IDOB $9 \angle=$
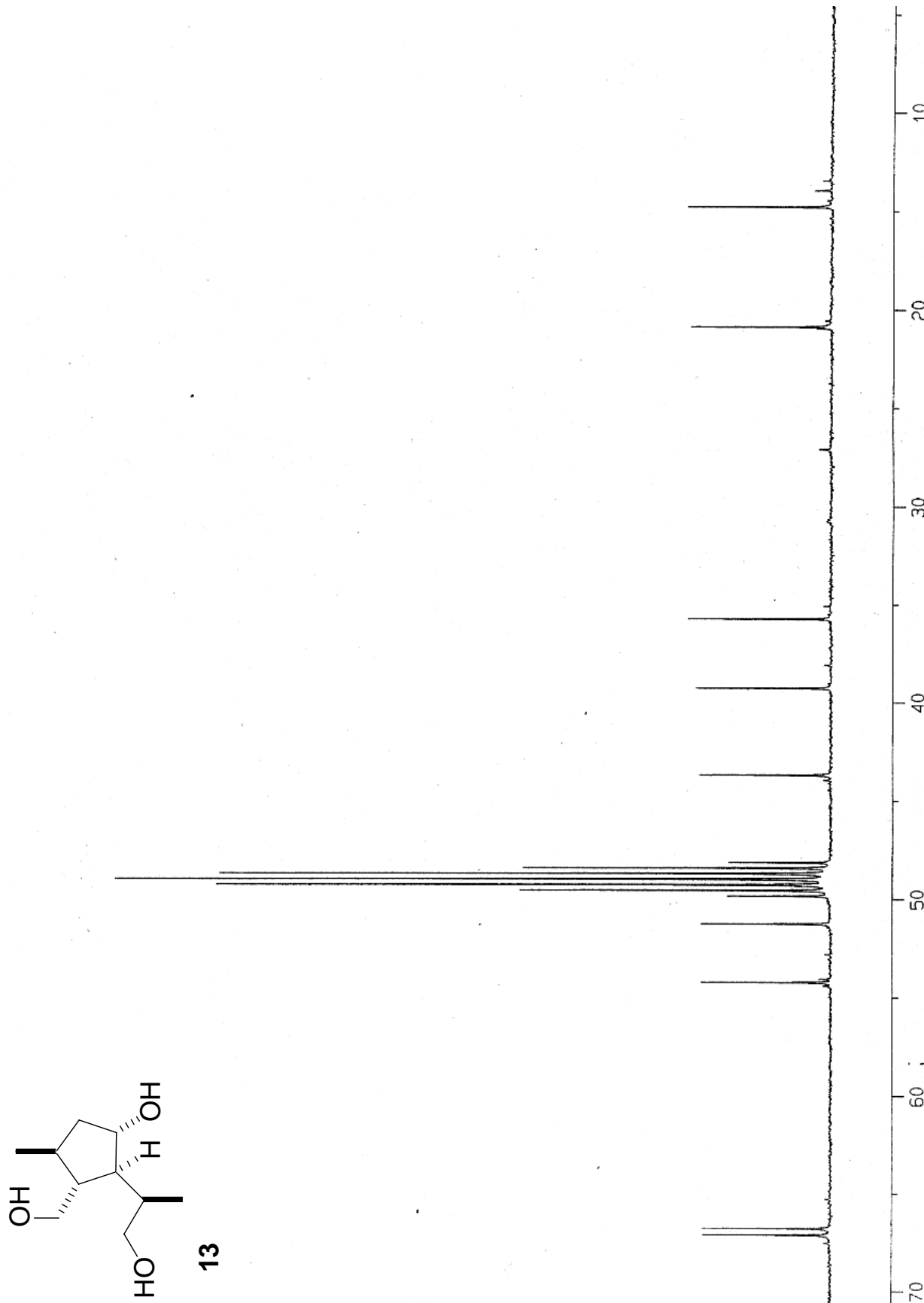


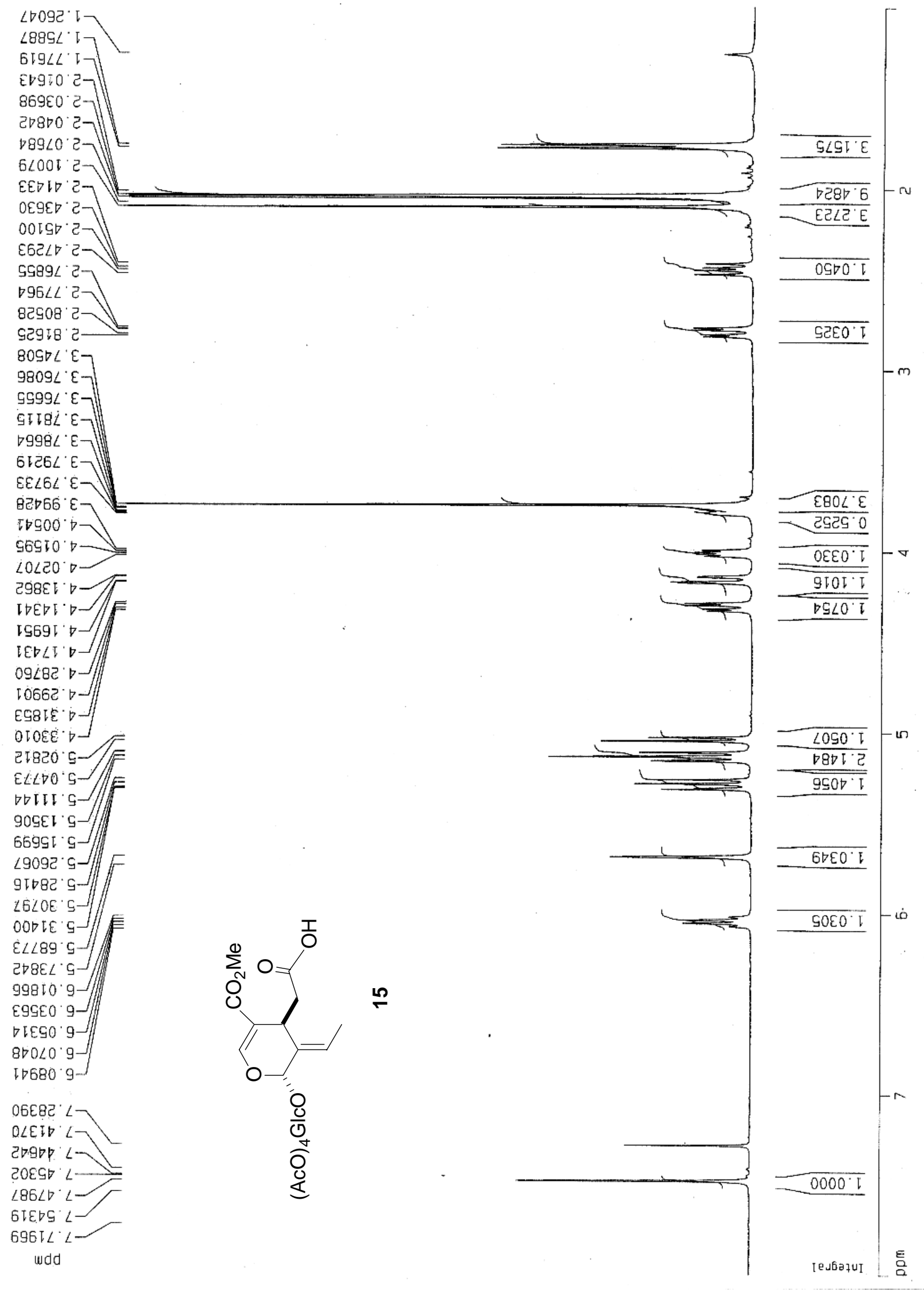



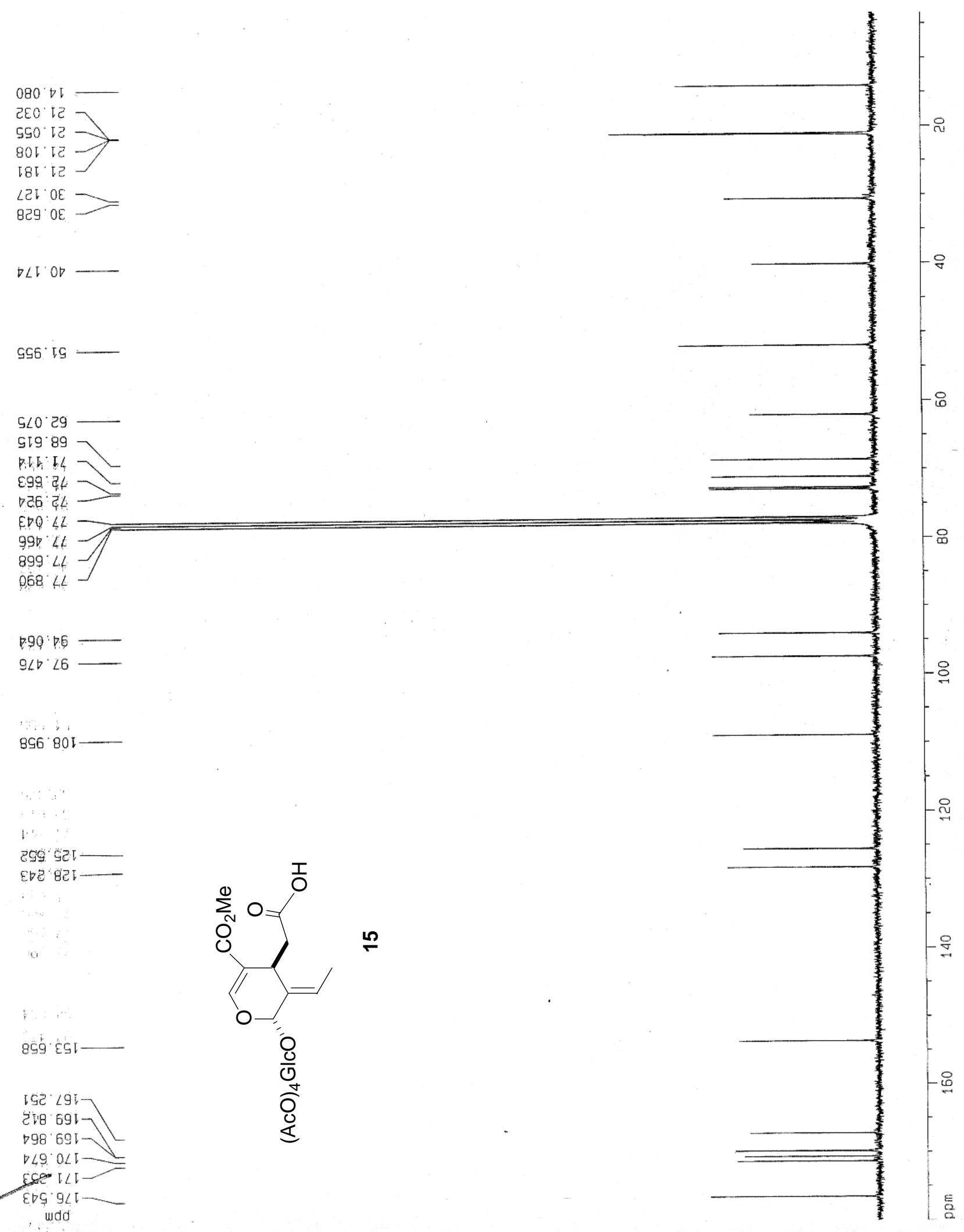


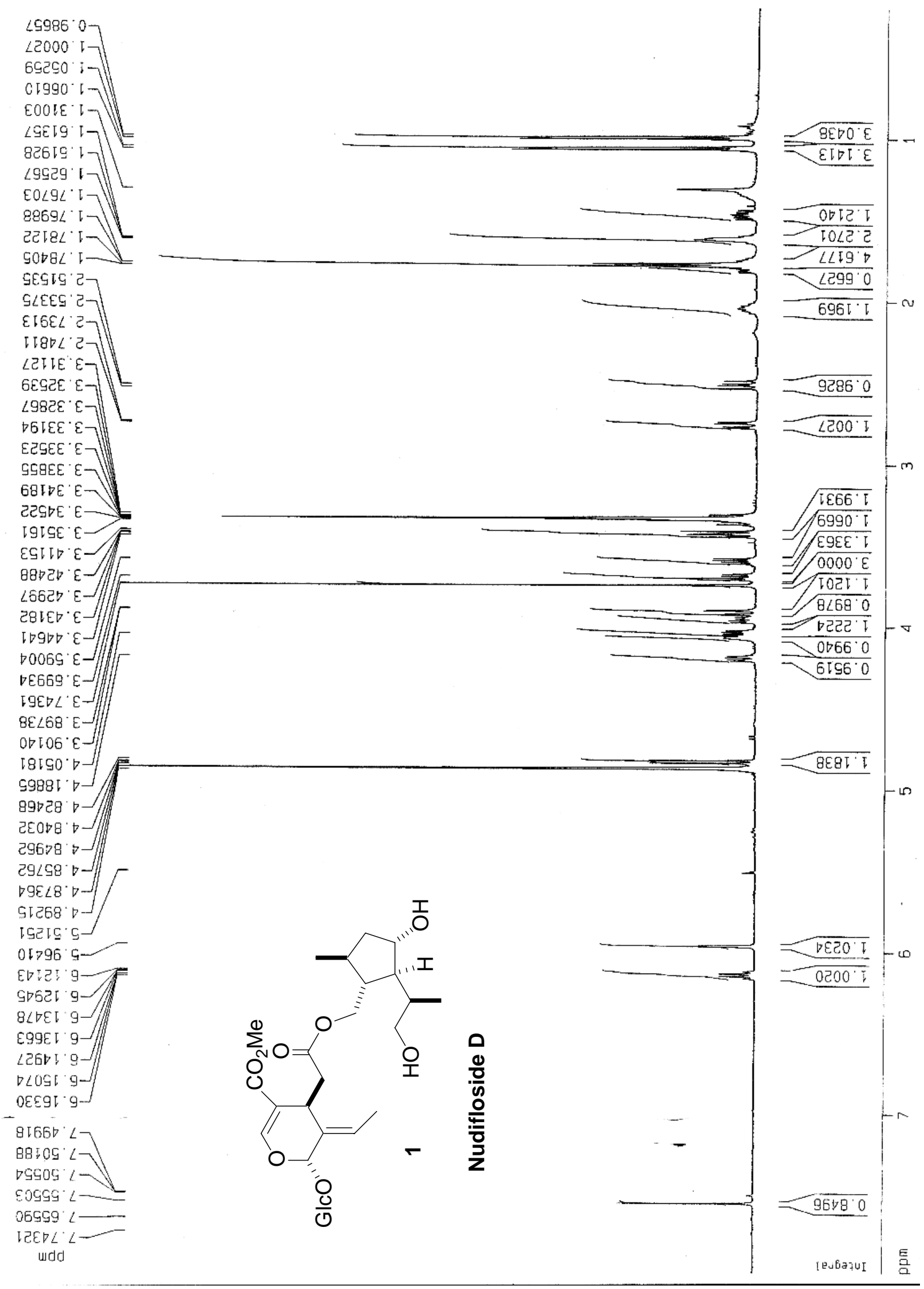




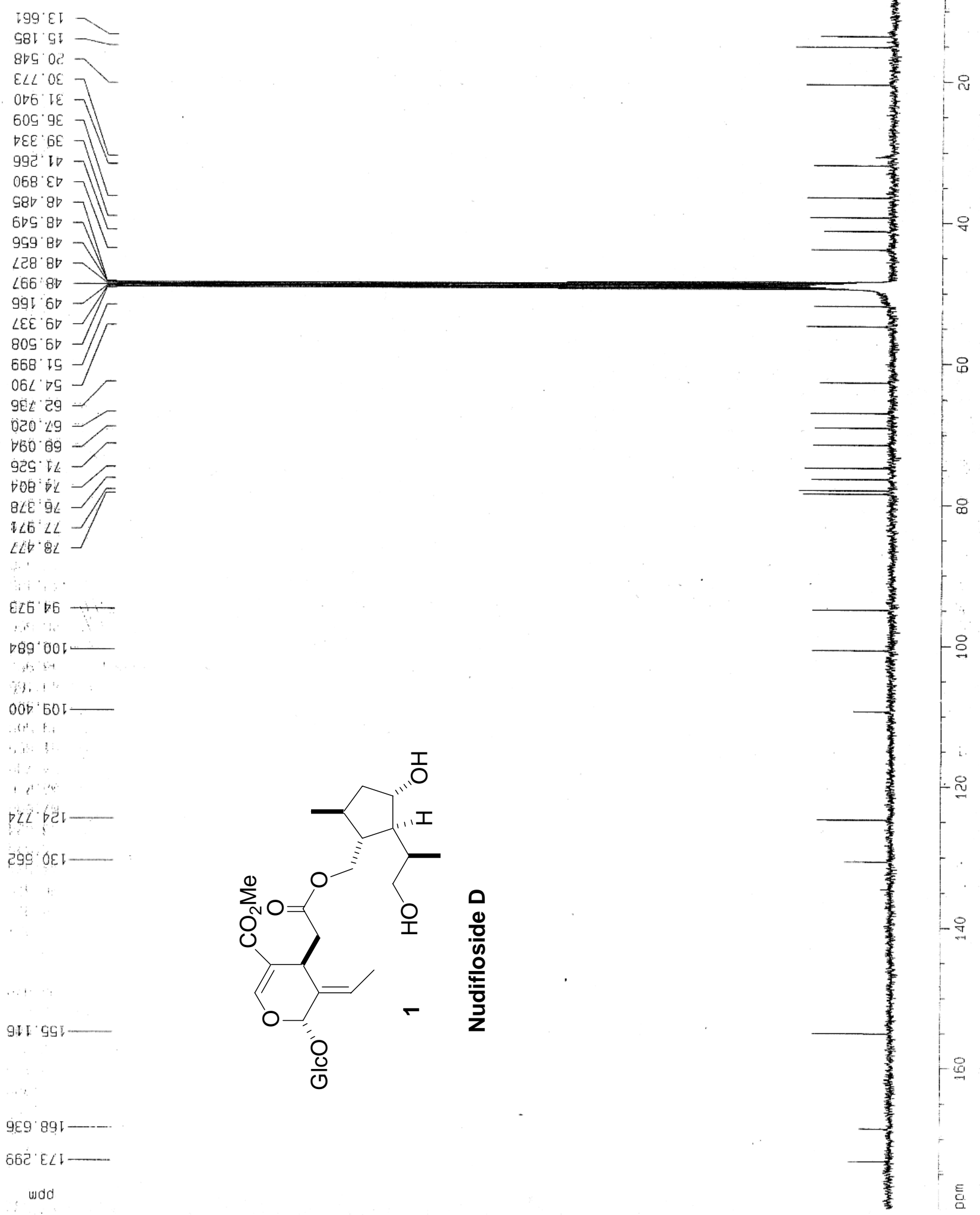




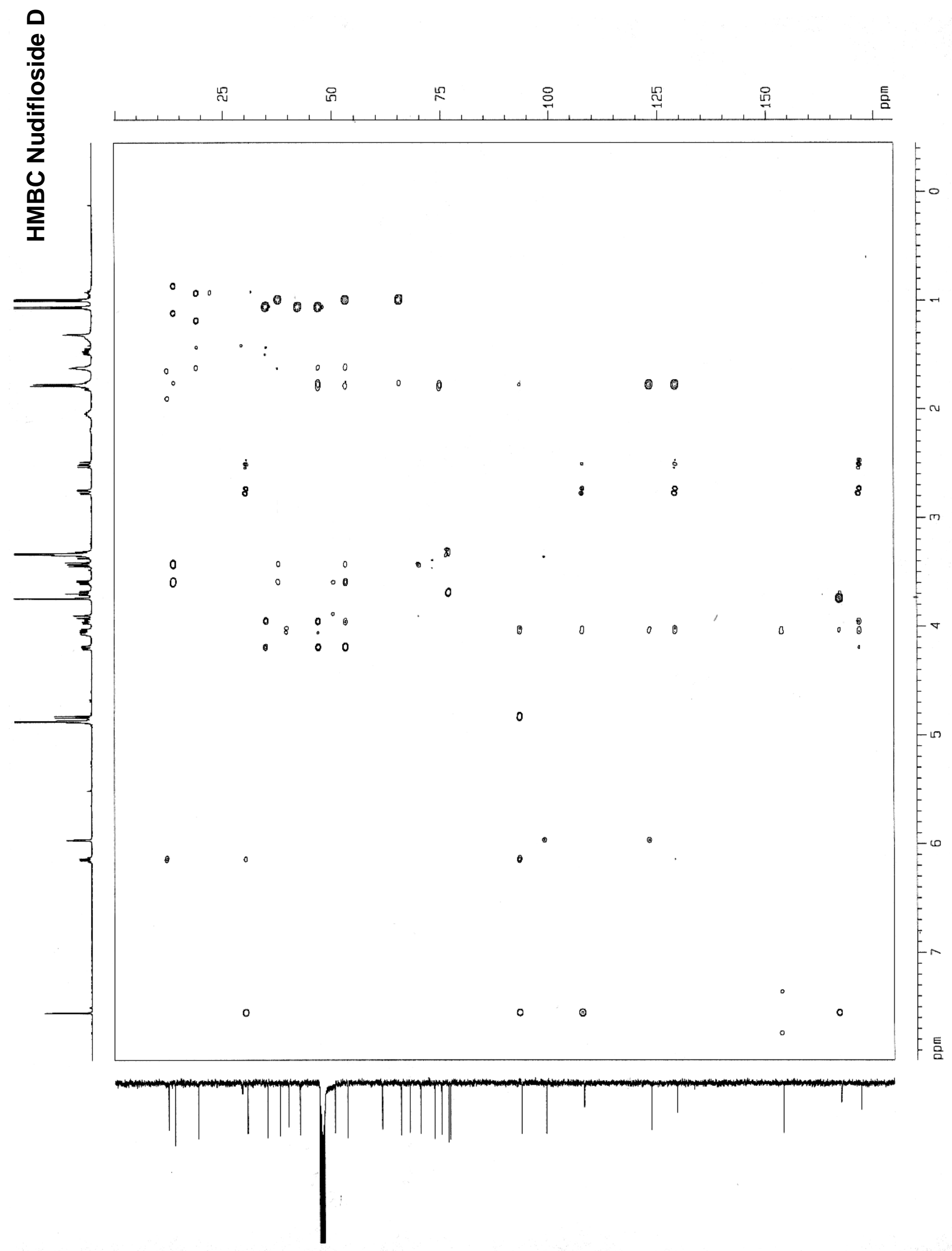




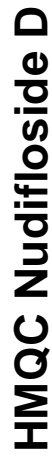

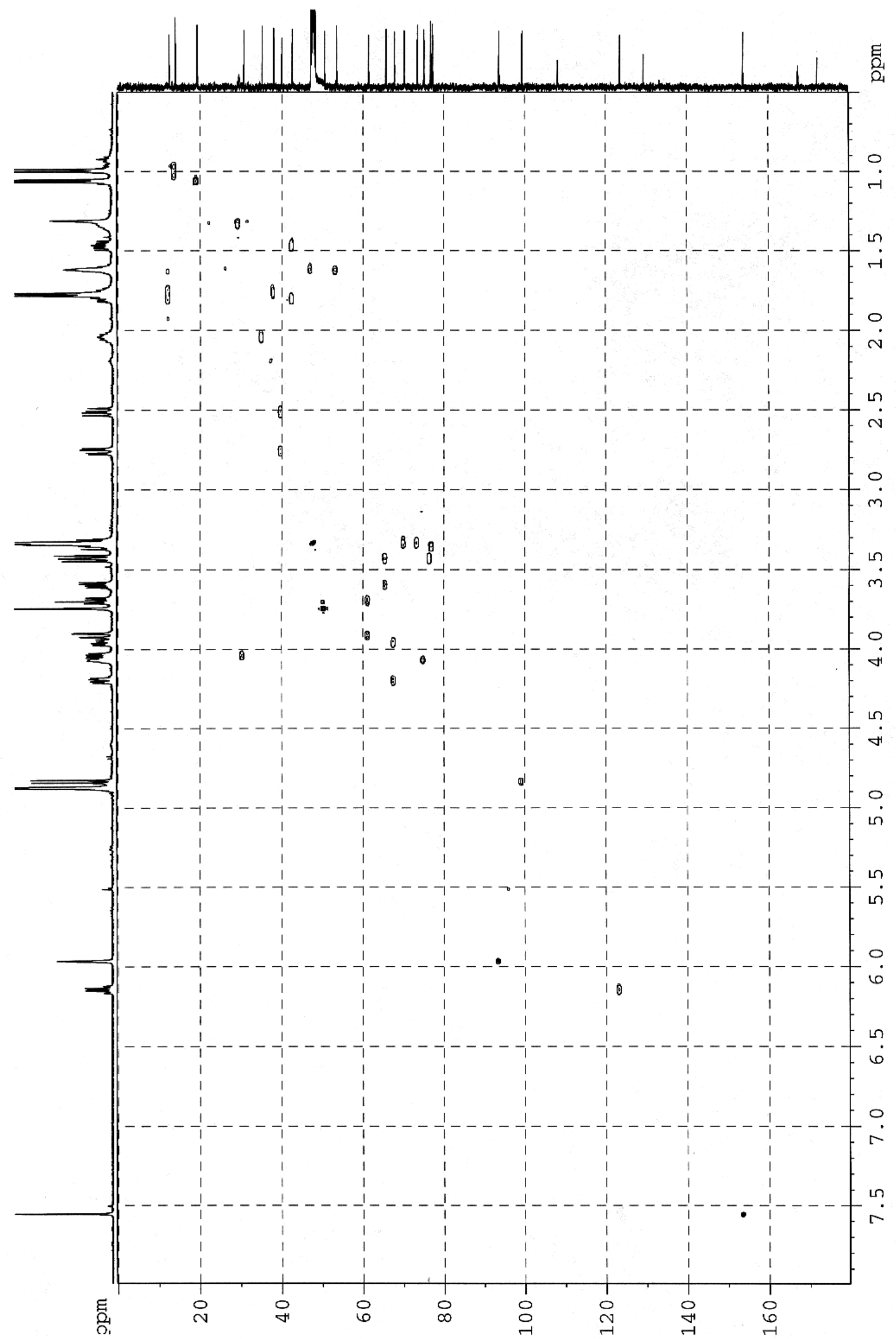




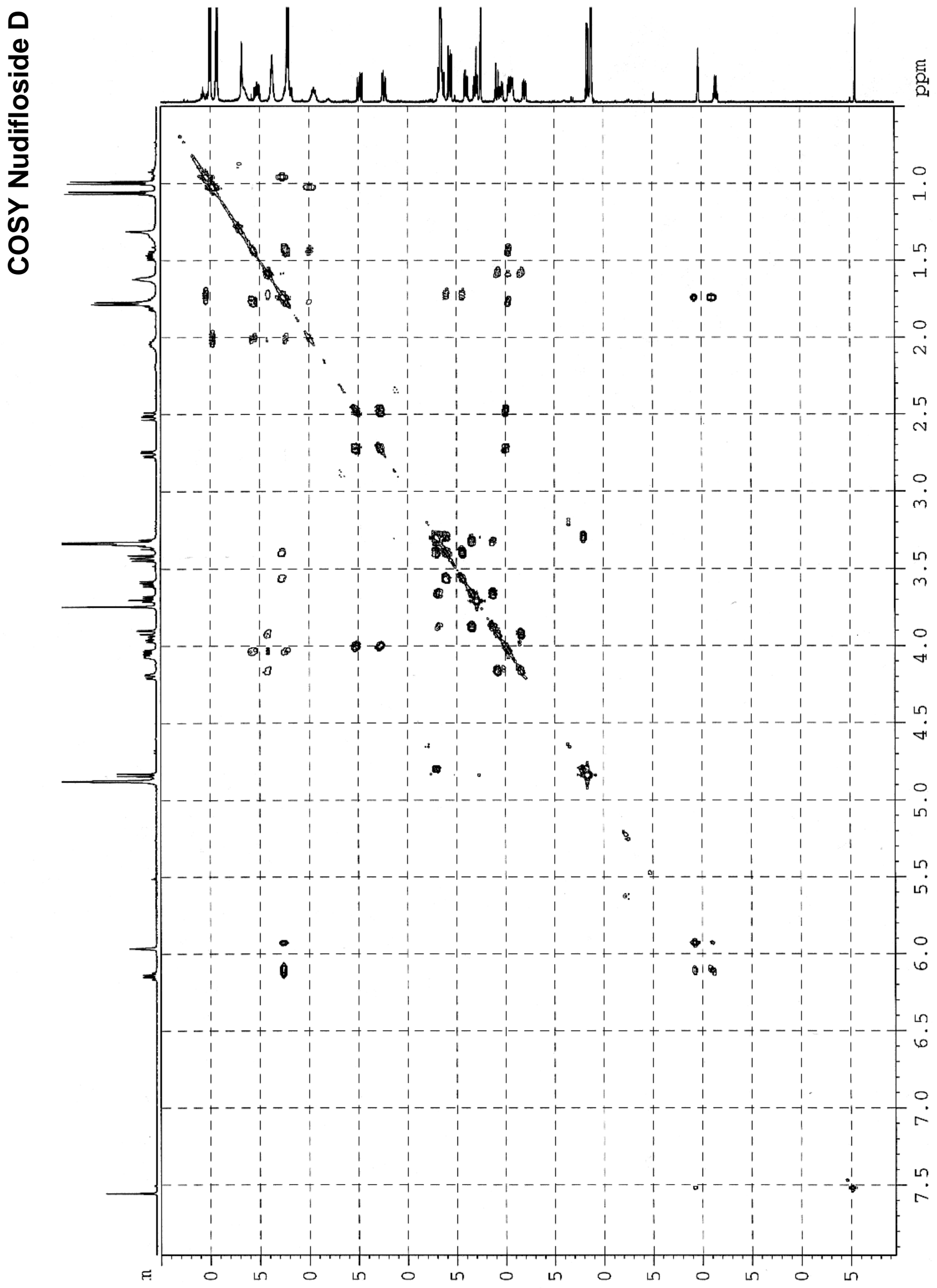




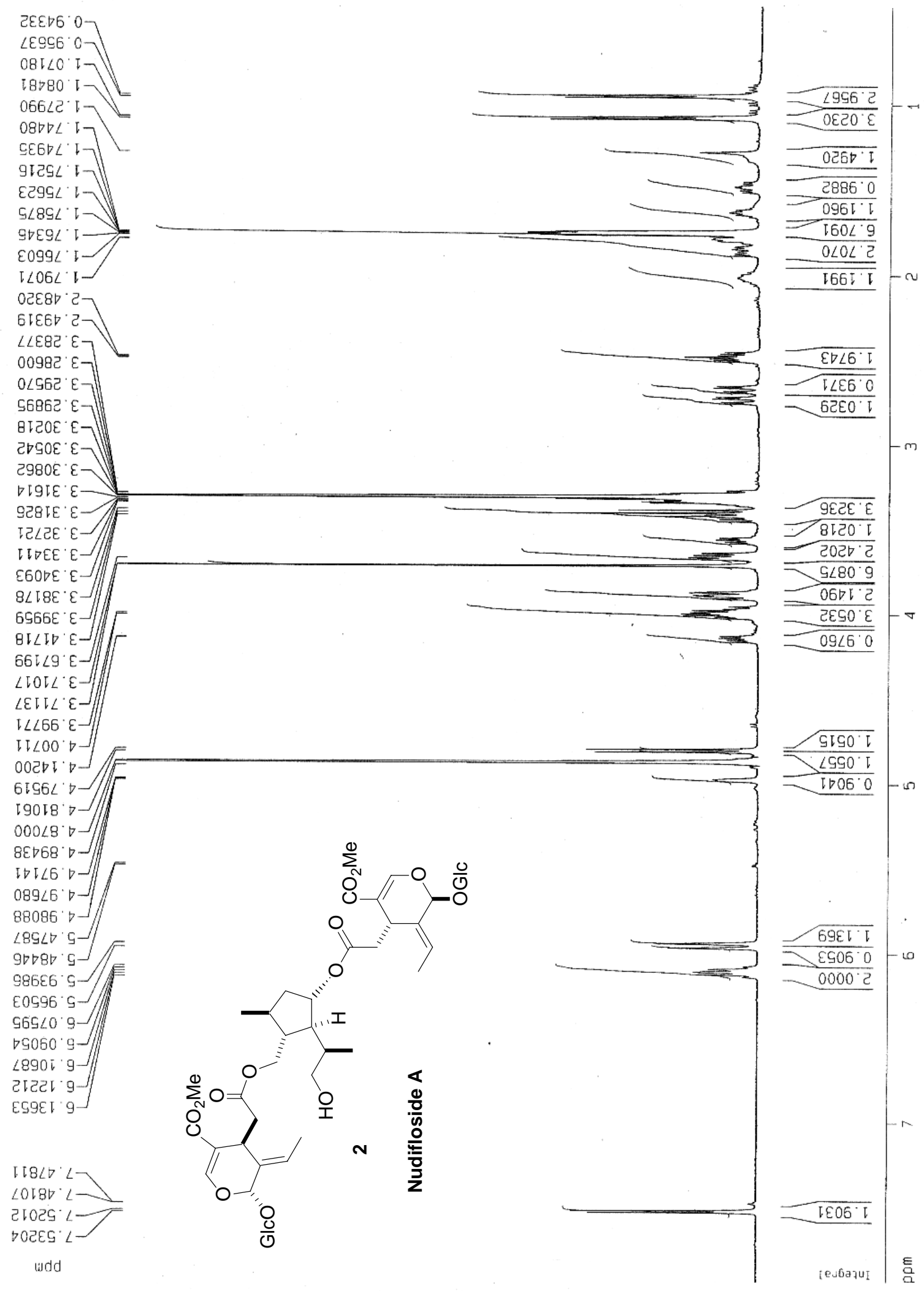




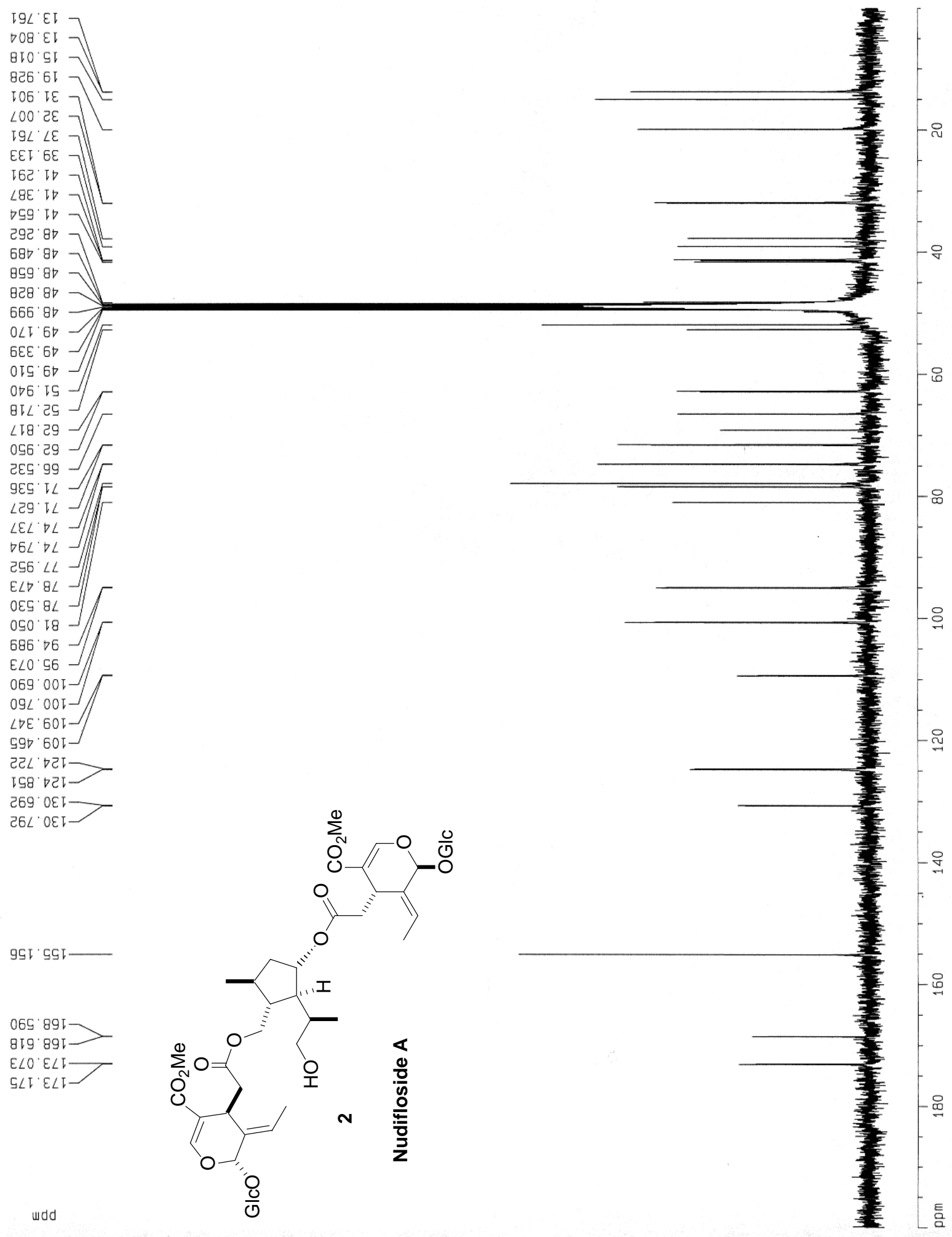




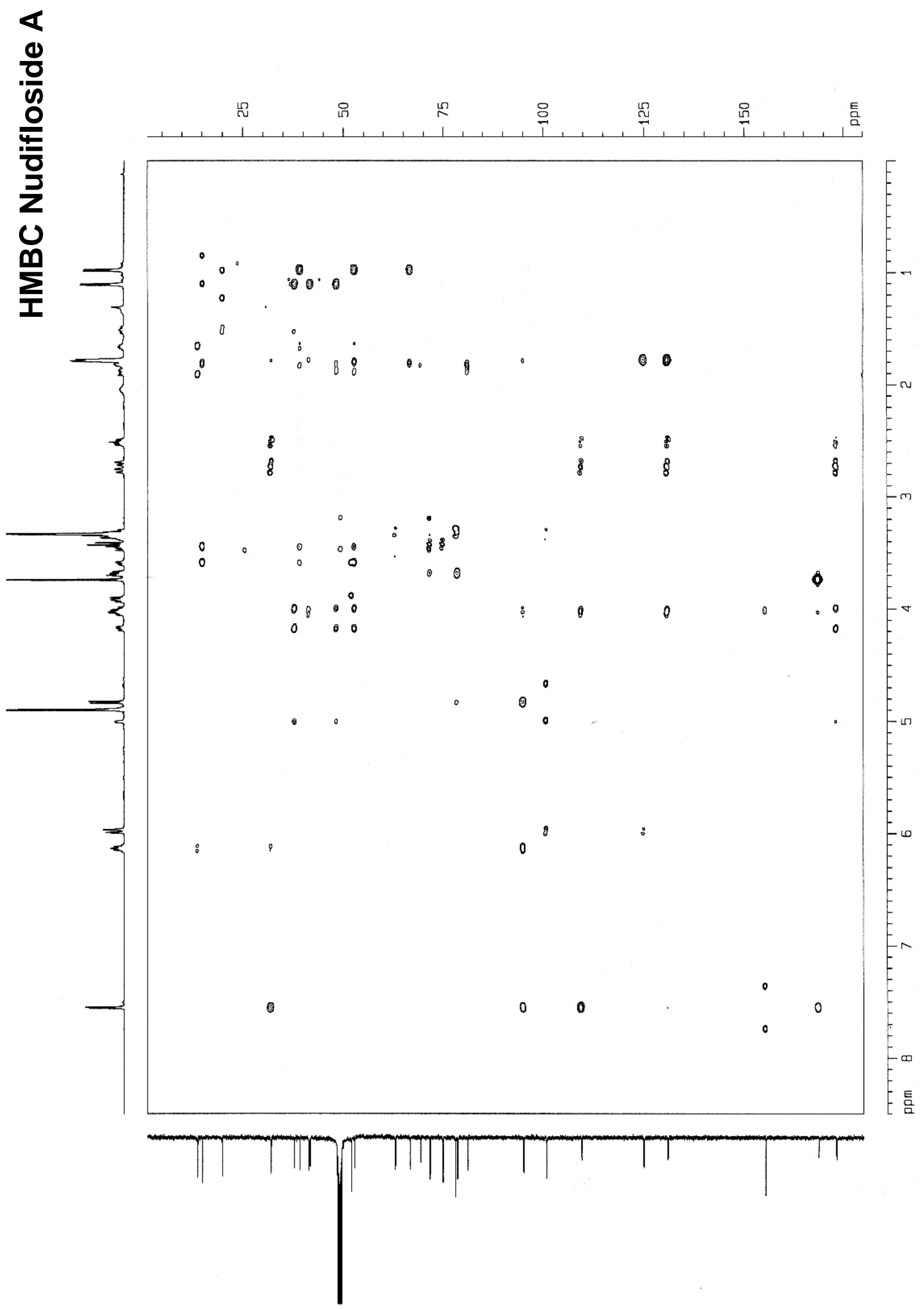




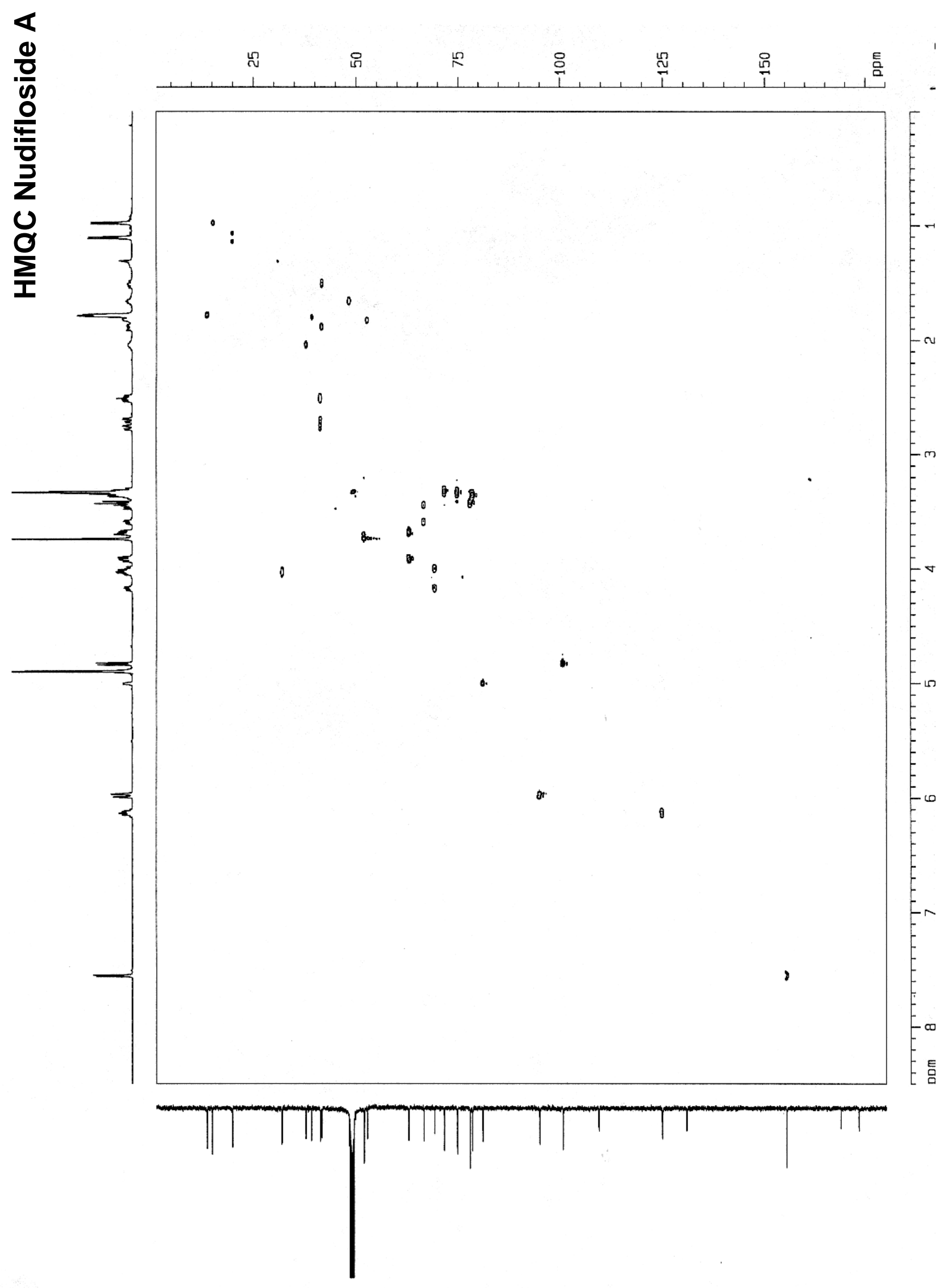




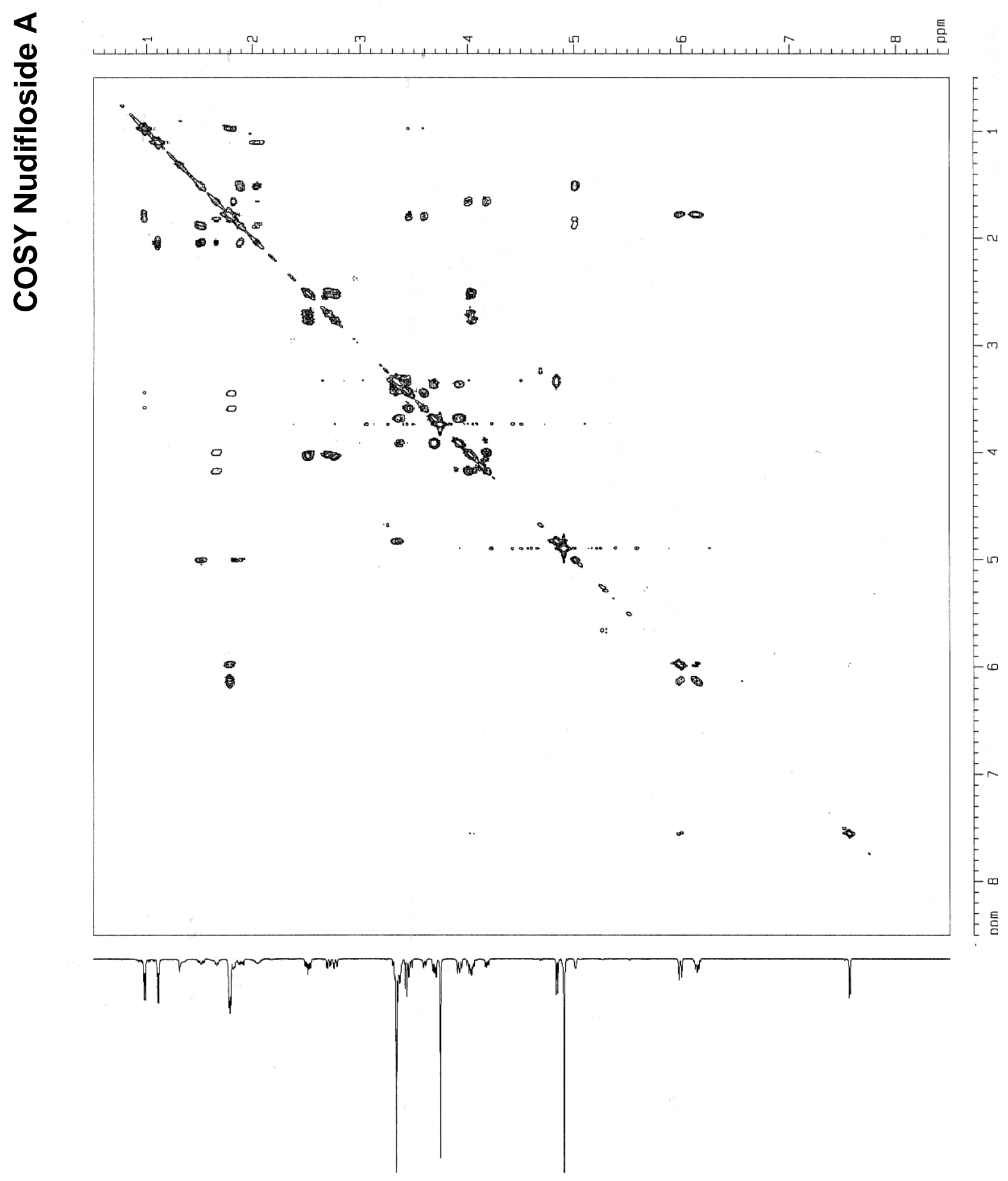


